

\section{$Q R^{\prime}$ \\ H4 6}

CORNELL UNIVERSITY THE

Floner Heterinary Jithrary FOUNDED BY ROSWELL P. FLOWER

for the use of the

N. Y. State Veterinary College 1897

This Volume is the Gift of Dr. V. A. Moore 


\section{DATE DUE}

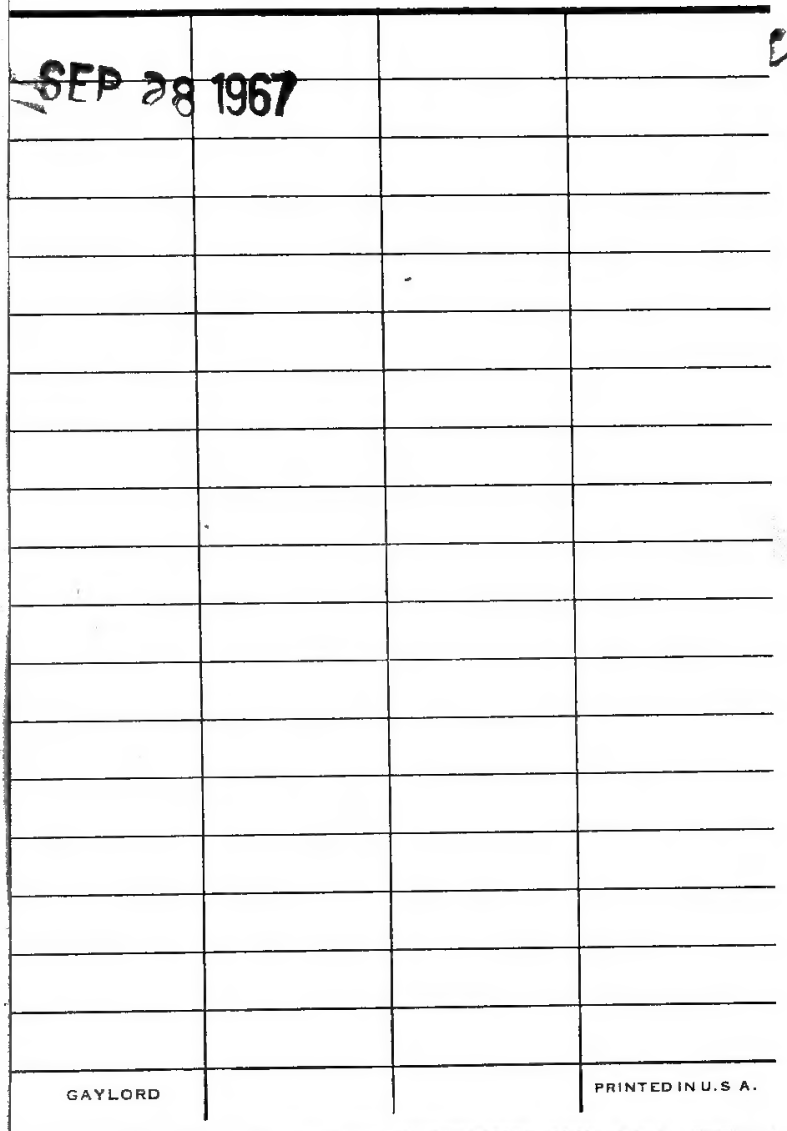

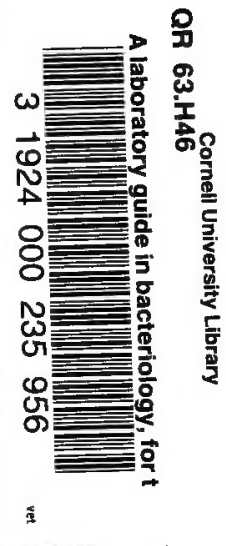




\section{Cornell University Library}

The original of this book is in the Cornell University Library.

There are no known copyright restrictions in the United States on the use of the text.

http://www.archive.org/details/cu31924000235956 


\section{A LABORATORY GUIDE IN BACTÉRIOLOGY}





\title{
A Laboratory Guide in Bacteriology
}

\author{
FOR THE USE OF \\ STUDENTS, TEACHERS, AND \\ PRACTITIONERS
}

BY

PAUL G. HEINEMANN, Sc.B.

Fellow in Bacteriology, the University of Chicago

CHICAGO

THE UNIVERSITY OF CHICAGO PRESS

1905 


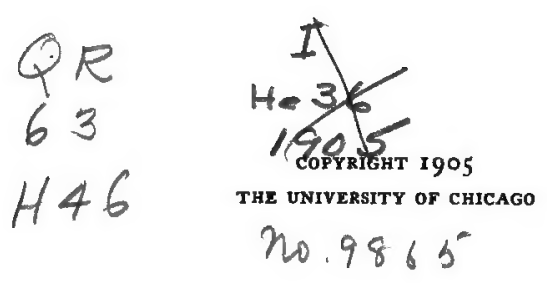




\section{PREFACE}

The considerations which led the writer to add this laboratory guide of bacteriology to the number of such guides already in existence were of various nature and may be briefly set forth here.

Probably no branch of biological science has advanced so rapidly during the past few years as the science of bacteriology, and it is difficult even for an active laboratory worker to keep abreast of this advance. A textbook or guide fixes the status of the science at the time of its writing, but almost before it leaves the press it becomes antiquated. Revisions, corrections, and additions are necessary at short intervals in order to keep a publication of this kind approximately up to date. There is therefore, almost at any given moment room for a new publication to fill the want of a progressive instructor for a guide that gives the latest accepted rules and practices of the laboratory. The value of such a publication will be enhanced by a plan and arrangement of sufficient flexibility and latitude to allow the instructor and the student to enter such additions and corrections as serve to bridge over the time between editions.

Medical students entering on a course in bacteriology often have had too little previous laboratory training in methods of precision. It is a matter of importance for the instructor to put himself in the attitude of mind of the student and to try to appreciate his difficulties in understanding details. Many of the pieces of apparatus employed in a bacteriological laboratory are novel even 
to the student trained in chemistry and biology, and it has been thought best to exhibit these, to the smallest detail, by means of illustrations-a feature not sufficiently considered in other guides.

The formulæ for stains and the methods of staining have not been collected in one chapter, as is usually the practice, because this tends to confuse the student. They are described during the progress of the course, as occasion offers to put them to practical use.

Culture-description charts have not been included in this volume. A beginner naturally makes incomplete descriptions and many alterations, and thus defaces the book and impairs its future utility. A sufficient number of loose charts perforated for binding should be furnished to the student at a nominal figure.

A point of inestimable importance is how best to stimulate the student to consult textbooks and special monographs, and other references, as often and as freely as possible. This guide has been written with the aim of not only not interfering in any manner with the reading, through including such points and characteristics as might make a textbook superfluous in the judgment of the inexperienced, but also of making it necessary for the student to read the best textbooks with freedom and understanding. Cultural and morphological features are left entirely to the actual observation of the student, supplemented by instruction and the reading of textbooks.

The course, as outlined, is identical with the medical course given at the University of Chicago, with a few additional chapters which may be used during courses for non-medical students. A chapter containing a fairly complete list of formulæ for culture media employed in 
advanced work has been added with the object of making them easily accessible to those engaged in advanced work.

The laboratory guides of Novy, Eyre, Frost, Gorham, Kanthack and Drysdale, and Connell, and the American edition of the Manual of Bacteriology of Muir and Ritchie, have been freely consulted. I take this occasion of expressing my deep gratitude to Professor Edwin $\mathrm{O}$. Jordan and Dr. Norman Mac Leod Harris for their invaluable help and suggestions in the preparation of this guide.

Chicago, Ill.

Paul G. Heinemann.

June, I905. 



\section{INTRODUCTION}

The advent of bacteriology into the realm of the biological sciences not only brought with it a new conception of the nature of many complicated phenomena, such as fermentation and disease, but also placed in the hands of experimental workers a new tool. The method of sterilization, of asepsis, made it possible for the first time to attack problems hitherto incapable of solution, or even of approach. This development of bacteriological technique, of rigid and undeviating adherence to definite rules and principles, is not likely to be passed over lightly by the historian of nineteenth-century science. The art of practical medicine and theoretical medical research alike owe much of their recent brilliant success to a ready adoption of the new method.

At the present time an active campaign is being set on foot by public health authorities against several widespread and serious diseases of the human race. In various parts of the world, malaria, tuberculosis, and typhoid fever are being fought energetically and with much success. In these systematic and organized movements the resources of bacteriology are being utilized as never before, and a full understanding of technical procedure and devices is deemed essential by all workers in this subject. The problems of water-supply and sewage disposal, 
of urban infantile mortality, and of the control of contagious diseases are all bound up with the intelligent application of bacteriological methods.

In the almost untilled field of industrial bacteriology there is need for a fuller appreciation of the value of bacteriological methods and principles. Many great industries are based wholly upon the proper selection and adaptation of micro-organisms, and a timely and discriminating utilization of their products. Loose and empirical methods have been in force in the past, but these must eventually give way to a more precise and truly bacteriological technique.

Agricultural bacteriology is just now much in the public eye, and it would be gratuitous to prophesy the results that may reasonably be anticipated in this direction. Here again crude, rule-of-thumb, "practical" ways of doing things are being supplanted by the scientific, the reasoning, and the precise.

To the student, whether in medical, hygienic, or industrial bacteriology, proper technical methods of work must always have a peculiar value, since without their aid advance is impossible, and stumbling and disastrous missteps are certain. A comprehensive outline of modern bacteriological methods, therefore, is a necessary adjunct to obtaining a true and full understanding of the underlying principles and tendencies of the science. The technique of 
bacteriology is one of its greatest contributions to both science and art, and the use of so valuable and simple a tool should be mastered not only by the biological teacher and investigator, but by practical workers in medicine, hygiene, and many other fields.

\author{
EDWIN O. JORDAN.
}





\section{TABLE OF CONTENTS}

Chapter I. Laboratory Rules . . . . . I

Chapter II. Cleaning, Preparing, and Sterilizing Glassware 8

Chapter III. Preparation of Culture Media . . II

Exercise I. Preparation of nutrient agar-agar II

Exercise 2. Preparation of glucose-agar I9

Exercise 3. Preparation of peptone-gelatin , 23

Exercise 4. Preparation of peptone-broth 25

Exercise 5. Preparation of potato . 27

Exercise 6. Preparation of litmus milk . 28

Chapter IV. Preparation of Staining Solutions . 30

Chapter V. The Microscope . . . . 31

Chapter VI. Collecting Bacteria from the Air . . 37

Chapter VII. Exercises on Infection and Sterilization 45

Exercise 1. Phenomena of infection * 45

Exercise 2. Phenomena of sterilization . . 45

Exercise 3. Phenomena of sterilization (continued) . 45

Chapter VIII. Study of Yeasts, Molds, and Torula . 49

Exercise I. Cultural studies . 49

Exercise 2. Study of the germination of spores $5 \mathrm{I}$

CHAPTER IX. Scheme for Routine Study of the Various

Groups of Organisms . . . 53

Chapter X. Method of Describing Cultures . 57

Chapter XI. Study of Certain Chromogenic Bacteria 69

Exercise I. Cultural studies . . . 69

Exercise 2. Study of pigments . . . . 7 I

Chapter XII. The Pyogenic Group • • • . 73

Exercise r. Subgroup A . . . . 73

Exercise 2. Subgroup B . . . . 76 
Chapter XIII. The Intestinal Group

Exercise I. Colon group

Exercise 2. Hog-cholera, Bac, enteritidis or intermediate group

Exercise 3. Typhoid-dysentery group

Exercise 4. Proteus group

Chapter XIV. The Capsulated Group

Chapter XV. The Diphtheria Group . . . 94

Chapter XVI. The Hemorrhagic Septicemia Group. $\quad 97$

Chapter XVII. The Anthrax Group . . . 98

Chapter XVIII. The Spirillum Group . . IoI

Chapter XIX. The Group of Acid-Resisting Bacilli IO3

Chapter XX. The Miscellaneous Bacteria . 105

Chapter XXI. The Actinomyces Group . .

Chapter XXII. The Anaërobic Group of Bacilli IO7

Chapter XXIII. Isolation of Unknown Bacteria from a

Mixture . . . .

Chapter XXIV. Bacteriological Examination of Water, Air, and Milk

Exercise I. Bacteriological examination of water II4

Exercise 2. Bacteriological examination of air $\quad$ I 8

Exercise 3. Bacteriological examination of milk

Chapter XXV. Influence of Disinfectants on the Growth of Micro-organisms . . . . 122

Exercises I and 2. Hill's test-rods 122

Exercise 3. Influence of sunlight 123

Exercise 4. Influence of moist heat . I24

ApPendix I. Special Media . . . . . I27

APPENDIX II. Staining Solutions . $\quad \mathbf{I 3}^{2}$

APPENDIX III. Frost's Culture Chart (modified) . $\quad$ I34

INDEX $\quad \cdot$. . . . . $\quad$ I39 


\section{CHAPTER I}

\section{LABORATORY RULES}

I. Carefully familiarize yourself with the laboratory rules. Upon their careful observance depend good work and your own safety.

2. Food must not be eaten in the laboratory; leadpencils, labels, or fingers must not be moistened with the tongue.

3. If any portion of a culture is spilled by accident upon the desk or floor, it should be immediately covered with a germicide $\left(\mathrm{HgCl}_{2}\right.$ I : I000 or 5 per cent. carbolic acid). After this has acted for Io or 15 minutes, wipe it up and throw the cloth or paper into a waste-jar.

4. In case the hands should by accident come in contact with infectious material, they should be washed with one of the above-mentioned germicides, and then thoroughly scrubbed with soap and water.

5. The platinum needles used in. making cultures should be sterilized in a flame shortly before and immediately after use, and before they are laid down. When the needles are covered with moist infectious material, they should be held at the side of the flame until dry before being sterilized; this will avoid the danger of scattering this material about the desk.

6. All possible care should be observed in the care of the apparatus, desk, etc. Solid materials must not be put into the sinks. Burned matches, paper, cotton, etc., are to be put in the crocks provided for that purpose, and not on the floor. 
7. Discarded cultures should be killed in the autoclav ( 5 minutes at $\mathrm{I}_{20^{\circ} \mathrm{I}}$ ) before emptying into the crocks.

8. When using the steam sterilizers and autoclav, see that the pan contains sufficient water before lighting the gas.

9. In general, see that the air inlets of Bunsen burners are open before lighting, and relight if the flame strikes down.

Io. Always return stock-bottles to the proper places on the shelves.

II. At the close of the day's work the desks should be washed off with corrosive sublimate, and the hands cleaned by thorough scrubbing with green soap and water.

I2. Before leaving the laboratory, see that the gas is shut off under all apparatus, that water faucets are closed, and that all glassware, etc., is replaced in the lockers.

\section{GENERAL DIRECTIONS}

I. After obtaining the key to the locker, examine the outfit, check the apparatus (Fig. I) on the furnished list, and return the same signed with your name. This signature is a receipt in full for the material received and in good condition, and the student is personally responsible for the return of the same in good condition.

2. Matches, peptone, gelatin, and filter-paper are furnished with the locker outfit in sufficient quantities to last through the course. Anything needed in excess must be furnished by the student.

I Degrees of temperature throughout this work are indicated according to the Centigrade system. 


3. To facilitate study, students may work in pairs in the preparation of culture media and the cultivation of organisms; but each student is responsible for a firsthand and independent description of each organism studied, as well as for stained cover-slip preparations.

4. Read carefully in the textbook and the laboratory guide the work for the day. This will greatly facilitate an intelligent and systematic plan of work.

5. Keep careful, neat, systematic notes of each exercise, and have them ready for inspection at any time. In the case of exercises devoted to the preparation of culture media, a simple statement as to their completion is sufficient.

6. Printed charts for description of cultures can be obtained on application to the laboratory assistant.

7. Accurate sketches must be made when called for by the directions.

8. All the notes pertaining to each exercise must be kept together. "University covers" of suitable size are recommended for this purpose.

9. Store clean cover-slips in a Stender dish and cover with $9^{6}$ per cent. alcohol. A soft linen cloth is necessary for wiping them. An ordinary clean handkerchief will do very well. Coarse material breaks them easily.

LOCKF:R OUTFIT

I50 culture-tubes.

Io potato culture-tubes.

6 fermentation-tubes.

I5 Petri dishes (for plate-cultures).

3 Erlenmeyer flasks, one 1000 c.c., two 500 c.c. each.

2 glass funnels, one 4 inches, one 6 inches.

4 bottles for staining-fluids, 
4 LABORATORY GUIDE IN BACTERIOLOGY

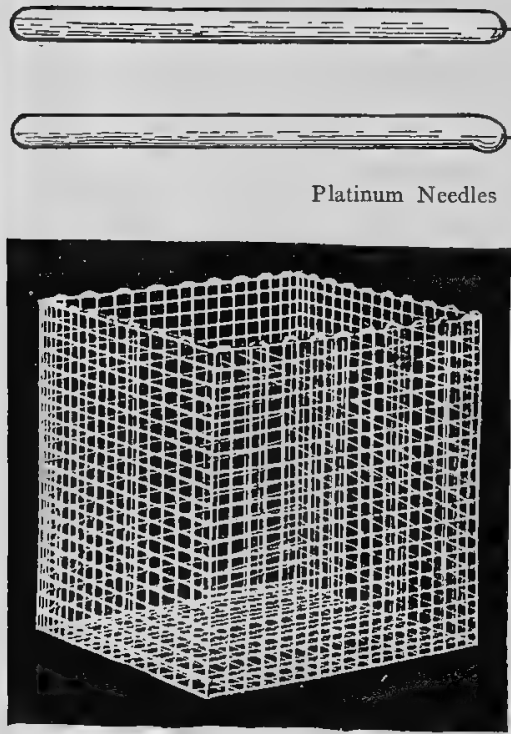

Wire Basket

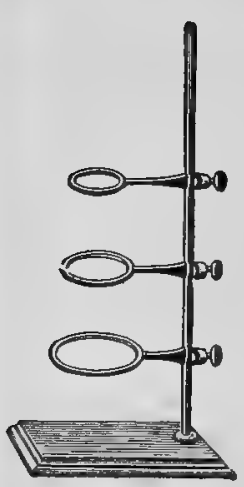

Retort-Stand

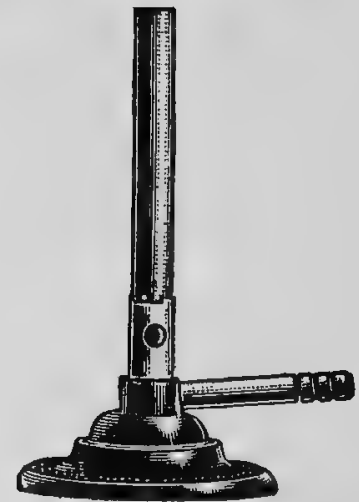

Bunsen Burner

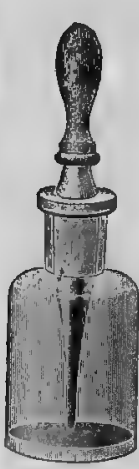

Bottle for Staining-Fluid
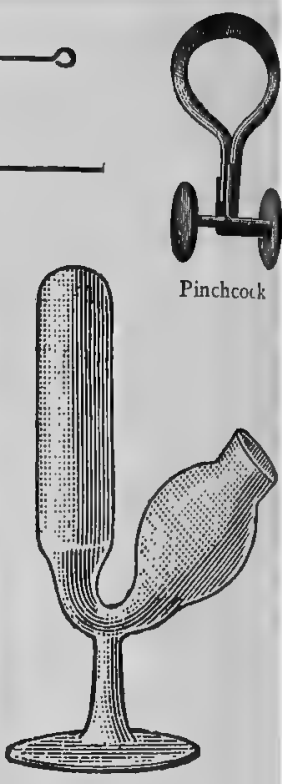

Fermentation-Tube
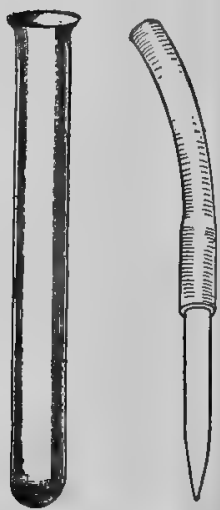

Culture-Tube 




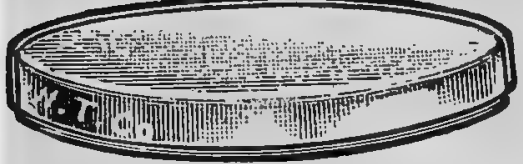

Petri Dish

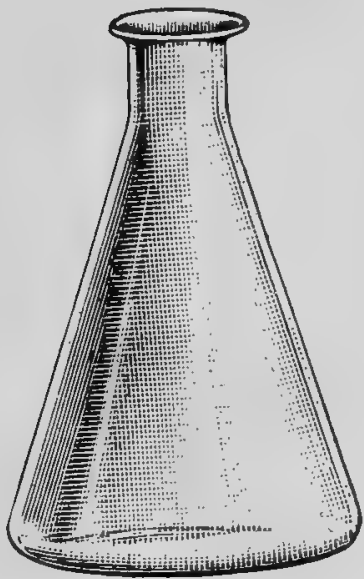

Erlenmeyer Flask

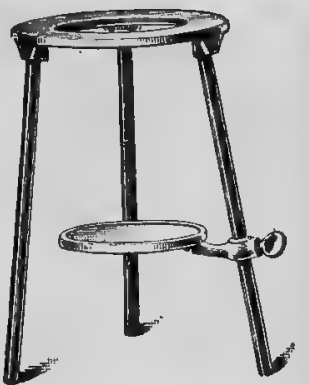

Tripod

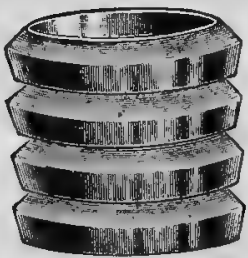

Watch-Glasses

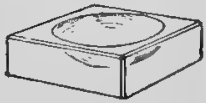

Seltcellar
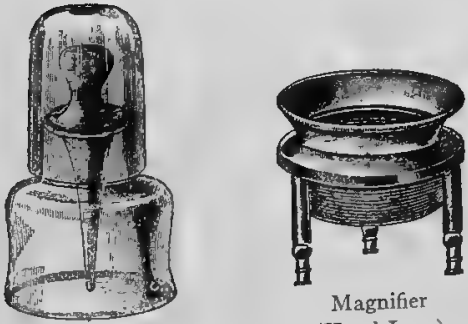

Magnifier

(Hand-Lens)

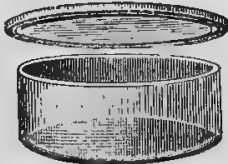

Stender Dish

F IG. I 
I balsam bottle.

I Stender dish (for cover-slips).

3 watch-glasses.

I saltcellar.

I glass rod.

2 platinum needles (turn the end of one needle around a sharp pencil point so as to form a closed loop).

8 tin cups or tumblers (cover the bottom of each with ordinary cotton).

4 wire baskets (to hold culture-tubes).

I Bunsen burner and rubber tubing.

I saucepan and cover.

3 graduates, one 500 c.c., one roo c.c., one ro c.c.

I pinchcock.

I pipette with rubber hose attached.

I magnifier (hand-lens).

I tripod.

I retort-stand with three rings.

I thermometer in case.

I box of matches.

40 grams of peptone.

I 20 grams of gelatin.

6 sheets of filter-paper.

I key.

This outfit may be changed and added to in the discretion of the instructor.

Obtain the following articles from the storeroom:

50 glass slides.

I camel's-hair brush.

50 round cover-glasses ( $18 \mathrm{~mm}$.).

2 towels (one should be boiled in dilute $\mathrm{NaOH}$, rinsed in clean water, and kept clean for wiping slides and cover-glasses.) 


I test-tube brush.

50 labels.

2 slide-boxes.

2 hollow-ground slides.

I glass pencil.

I pair of forceps.

I, or better 2, pairs of cover-slip forceps. 


\section{CHAPTER II}

CLEANING, PREPARING, AND STERILIZING

\section{GLASSWARE}

EXERCISE I. CLEANING GLASSWARE

Culture-tubes, flasks, fermentation-tubes, and Petri dishes must be free from organic matter, acids, and alkalis. They are best cleaned as follows:

a) Completely immerse them in a vessel containing soapsuds or soap-powder, boil for at least io minutes, then thoroughly brush them with the tube-brush; or, immerse them for an hour or more in the chromic-acid cleaning-mixture, consisting of-

Potassium dichromate............. 60 parts

Water........................ 300 parts

Concentrated sulphuric acid..........460 parts

The sulphuric acid is to be added slowly with constant stirring.

b) Rinse thoroughly in tap-water.

c) Again use the tube-brush, and soap and water, if necessary.

d) Rinse very thoroughly, and get rid of every trace of acid, soap, or alkali.

e) Turn upside down in a basket, and heat for 20 minutes in the hot-air sterilizer until perfectly dry.

EXERCISE II. PLUGGING OF CULTURE-TUBES

Directions for plugging.-Take a small amount of ordinary, non-absorbent cotton of good quality, fold it once, and by means of a glass rod push it into the mouth 


of the tube to the depth of about $\frac{3}{4}$ of an inch; or roll the cotton into cylinders of the same diameter as the culure-tube and insert it into the mouth (Fig. 2). The plug should allow the interchange of air, and at the same time be tight enough to hold the weight of the tube, and with no crevices on the side. The glass rod should never be pushed through the cotton.

EXERCISF, III

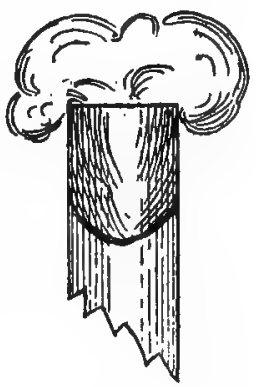

Fig. 2

Plugged Culture-Tube

STERILIZATION OF GLASSWARE

Sterilization is the process of killing all micro-organisms. This may be accomplished by heat, by certain chemicals, or by filtration. Sterilization by heat may be accomplished--

I. By dry heat; this method is applied to sterilization of many kinds of apparatus.

2. By moist heat; this method is applied largely to

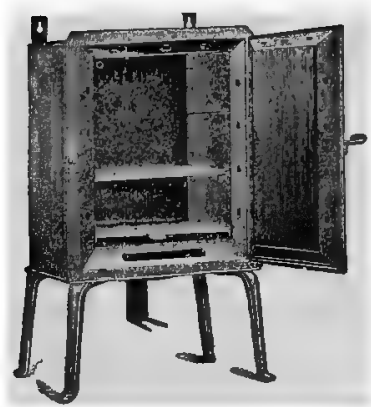

Fig. 3 sterilization of culture media, and will be described in Chap. III, Exercise II.

Hot-air sterilizers are boxes with double walls of sheetiron. The bottom shelf should always be covered with a piece of sheet-asbestos, to prevent too rapid heating of the apparatus. By means of a Bunsen burner with three Koch's Hot-Air Sterilizer flames a heat of about $150^{\circ}$ may easily be maintained. The flames of the burner 
should enter the hole provided at the bottom of the sterilizer for that purpose. Care should be taken to avoid the possibility of the flame becoming luminous, as this would fill the box and cover the glassware with soot (Figs. 3 and 4).

The plugged culture-tubes are then placed in the

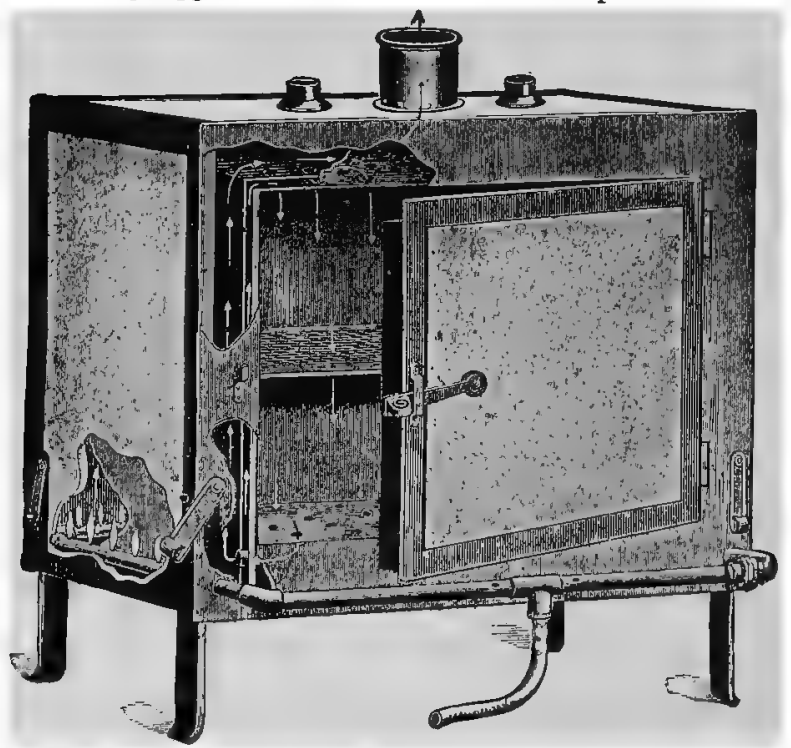

Fig. 4

Lautenschlager Hot-Air Sterilizer

sterilizer, and a temperature of $150^{\circ}$ is maintained for about 30 minutes, or until the plugs are slightly charred. This can be recognized by a slight brownish color. The tubes are now not necessarily sterile, but the plugs have become set so as to fit the mouth of the tube, and may easily be removed and replaced. 




\section{CHAPTER III}

\section{PREPARATION OF CULTURE MEDIA}

Each student is required to make up the following amounts of culture media:

\begin{tabular}{|c|c|c|}
\hline Name of Medium & Amount & Number of Tubes \\
\hline 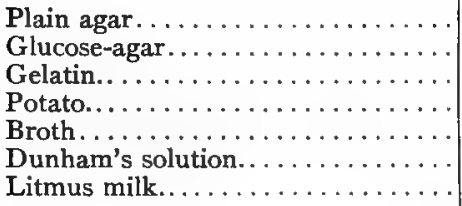 & $\begin{array}{l}1000 \mathrm{~g} . \\
250 \mathrm{~g} . \\
300 \mathrm{~g} . \\
300 \mathrm{c.c} . \\
300 \mathrm{c.c} . \\
300 \mathrm{c.c} .\end{array}$ & $\begin{array}{l}30 \\
20 \\
25 \\
10 \\
25 \\
25 \\
25\end{array}$ \\
\hline & & I6o \\
\hline
\end{tabular}

EXERCISE I. PREPARATION OF NUTRIENT AGAR-AGAR

I. Weigh the saucepan without the lid accurately and make note of the weight.

2. Measure into the saucepan 1000 c.c. of tap-water, adding about 300 c.c. of water to allow for evaporation, and heat over gas.

3. Cut and shred $15 \mathrm{~g}$. of agar-agar ( $1 \frac{1}{2}$ per cent.), add to the water, and boil slowly, with constant stirring, until perfectly dissolved, taking care not to overheat.

4. Add $3 \mathrm{~g}$. of extract of beef. When dissolved, remove the saucepan from the flame, and

5. Slowly dust Io (I per cent.) g. of Witte's peptone on the surface, constantly stirring until perfectly dissolved. Avoid allowing the peptone to clump.

NotE.-Agar-agar (called simply "agar") is a watery extract of certain seaweeds found on the Pacific coast of Asia. 
6. Adjust the reaction.

Bacteria, especially pathogenic bacteria, grow preferably in a medium which is neutral or slightly acid to phenolphthalein (alkaline to litmus). Extract of beef always contains a certain amount of acid, which has to be neutralized. Two methods may be employed to accomplish this purpose.

First method.-Gradually add a 4 per cent. soution of sodium hydrate $(\mathrm{NaOH})$ until a sample on the end of the glass rod turns phenolphthalein paper a pale rosepink color. If accidentally too much $\mathrm{NaOH}$ is added, it may be readjusted by means of a 5 per cent. solution of hydrochloric acid (HCl). This method is sufficiently accurate for ordinary purposes. The medium is then strongly alkaline to litmus.

Second method.-A more precise method is as follows: By means of a pipette measure 5 c.c. of the liquid into a porcelain evaporating-dish; add 45 c.c. of distilled water and I c.c. of a solution of I part phenolphthalein in 200 c.c. alcohol ( 50 per cent.), then heat to the boilingpoint and slowly add from a graduated burette enough $\frac{1}{20}$ normal $\mathrm{NaOH}$ to neutralize. (The liquid must have a decided, stable, pale-pink color which does not vanish when heated.) Read the amount used from the burette, and calculate the amount of normal $\mathrm{NaOH}$ needed for Iooo c.c. Then add 0.5 per cent. normal $\mathrm{HCl}$.

Example.-By reading the burette we find that it takes I. 2 c.c. $\frac{1}{20}$ normal $\mathrm{NaOH}$ to neutralize 5 c.c. of the medium, diluter with 45 c.c. of distilled water. To neutralize I liter, it will take 200 times this amount, which amounts to 240 c.c. of the $\frac{1}{20}$ normal $\mathrm{NaOH}$. Divide 240 by 20 , and the result (I 2 c.c.) is the amount 


of normal $\mathrm{NaOH}$ needed to neutralize I liter of medium. To this add 5 c.c. normal $\mathrm{HCl}$.

The medium is then 0.5 per cent. acid to phenolphthalein ( 0.5 per cent. Fuller's standard), or fairly strongly alkaline to litmus.

The same result may be obtained by deducting 5 c.c. from the amount of normal $\mathrm{NaOH}$ to be added per liter. If, therefore, in the above example I 2 c.c. -5 c.c. $=7$ c.c. normal $\mathrm{NaOH}$ is added, the resulting reaction of the medium is 0.5 per cent. acid without the addition of $\mathrm{HCl}$.

A normal solution is the equivalent weight in grams (gram-molecule) of a chemical in rooo c.c. of distilled water. In the case of monovalent elements combining to form the chemical, the molecular weight of the latter is taken; in the case of bivalent ones, the molecular weight is divided by 2 ; etc.

7. Allow the liquid to cool to $60^{\circ}$; test by placing the thermometer in it.

8. While cooling, dissolve the whites of two eggs in 75 c.c. of water, and stir well into the liquid while still at $60^{\circ}$. The egg albumen is added for the purpose of clarifying the liquid. If added before the temperature has fallen to $60^{\circ}$, it would partially coagulate, and thus not serve the purpose. On the other hand, if allowed to cool to about $40^{\circ}$, the agar would solidify. Upon gradually heating the medium the albumen coagulates, mechanically incloses suspended particles, carries them to the top, and forms a superficial film.

9. Heat again, without stirring, on a piece of asbestos over the flame. A film will gradually form and harden with a dry surface.

Io. Now adjust the weight by calculating the total 


\section{I4 LABORATORY GUIDE IN BACTERIOLOGY}

weight of the different ingredients, allowing rooo g. for all the water used and $30 \mathrm{~g}$. for the weight of the white of each egg and the weight of the pan. If the actual total weight is in excess of the amount calculated, boil again

Frg. 5

Apparatus for Filtering Media
a. Filter
ঠ. Large funnel
c. Small funnel
d. Rubber hose
c. Pinchcock
f. Pipette
g. Culture-tube

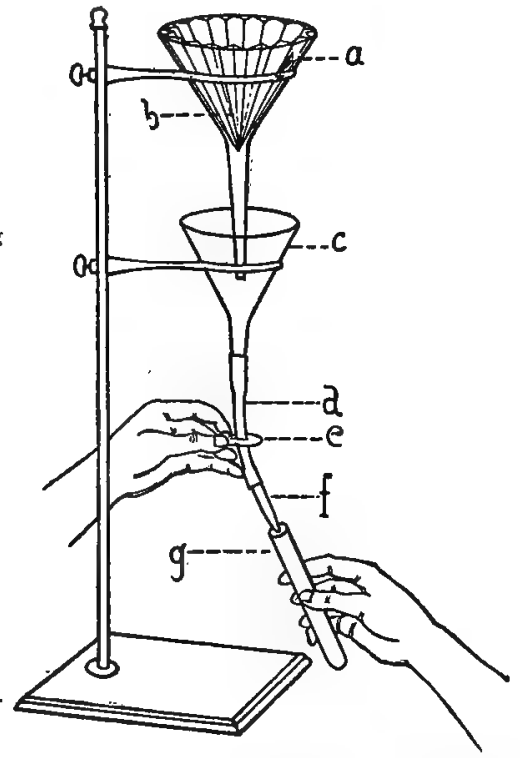

until the proper weight is reached. If, on the other hand, the total weight is less, make up the deficiency by adding water.

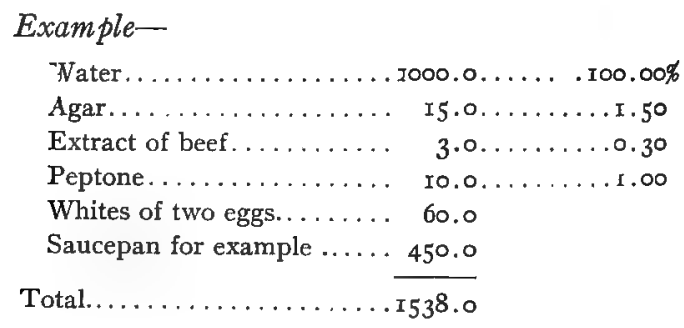




By placing weights to correspond to this amount on the left side of the scales, and the saucepan with contents on the other side, the weight can be readily adjusted.

II. Heat again to the boiling-point, and filter the liquid into culture-tubes to about one-third of their length ( 7 c.c.).

Method of filtering media.-Arrange two funnels, a pipette with hose, and a pinchcock on the retort-stand, as illustrated in Fig. 5. Place a strainer made of perforated tin (Fig. 6) against the side of the saucepan,

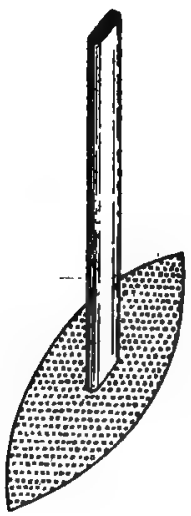

Fic. 6

Strainer

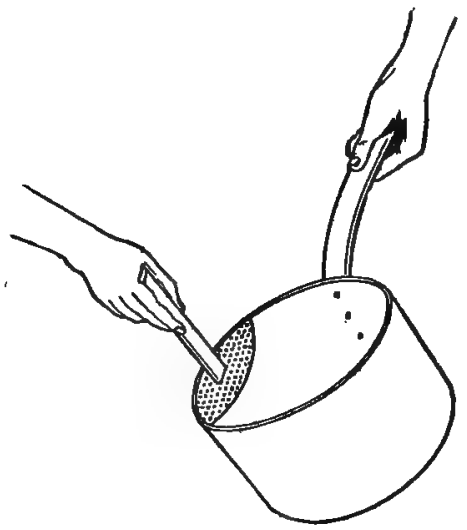

FIG. 7

Strainer in Position

near the top, as illustrated in Fig. 7 , and pour the medium into the larger funnel holding the filter.

For the purpose of filtering the medium either white filter-paper of the best quality or absorbent cotton may be used. A filter-paper folded in the following manner is very serviceable:

I. Take a square piece of filter-paper twice as wide 
as the depth of the funnel to be used (Fig. 8, a), and fold to half the size (Fig. $8, b$ ) so as to make $x$ cover 2 , and 3 cover 4

2. Fold this to make I cover 2 and 3 cover 4 (result: Fig. 8, c). It consists of four layers and forms a square.

3. Fold the upper part, consisting of two layers,
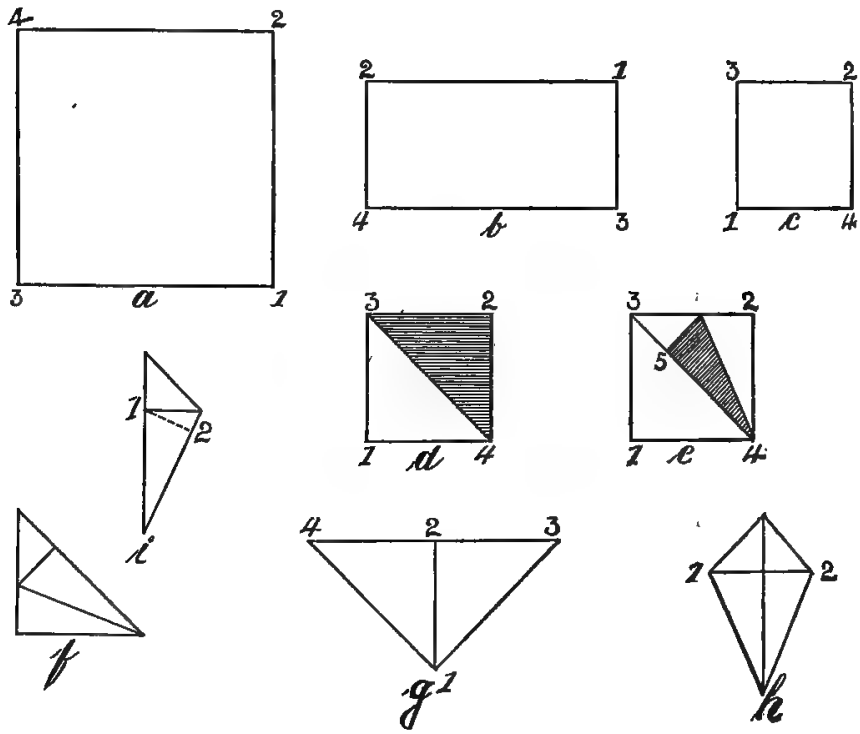

Fig. 8

Method of Folding Paper Filtcr

(For reference letters see text)

from I to 2 (Fig. 8, d). The shaded triangle, 2-3-4, now has six layers; the other $1-3-4$, two layers.

4. Fold the upper double layer so as to make 2 cover a point in the diagonal at 5 , taking care to make a sharp 


point at 4 (result: Fig. $8, e$ ). The shaded part is now eight layers deep.

5. Turn the folder face down, and repeat the operations exactly as in 3 and 4 (result: Fig. $8, f$ ).

6. Take up and open the large middle fold (result: Fig. $8, g$ ). The two halves must now be symmetrical.

7. Fold so as to make the lines $\mathrm{I}-3$ and I-4 meet at the center line I-2 (result: Fig. $8, h$ ).

8. Now pick up and fold backward so as to have I cover 2 in the back (result: Fig. 8, $i$ ).

9. Cut through the line $I^{-2}$ and open up. The extreme ends will be found without a fold, and may be folded so as to make nine sharp edges.

This filter is then evenly inserted into the funnel, spreading the folds at a distance from each other as nearly alike as possible. Great care should be taken to make the folds and the point sharp, as this insures rapid filtering of the liquid and prevents the filter from tearing. A wire rack may be used, but does not yield nearly so good results (Fig. 9).

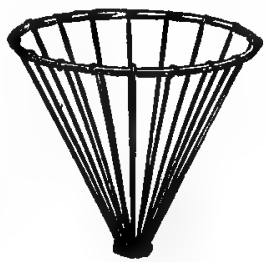

FIG. 9

Wire Rack

Another method, simple and yielding excellent results, is the use of absorbent cotton. Take a piece about 3 inches square, and cover lightly the lower end of the funnel with it. Take a larger piece, also square, judging the size according to the size of the funnel; pull it gently, so as to make the layer of cotton thinner, without showing ahy open spaces; and spread this around the inner surface of the funnel.

If a vacuum pump is available, the medium may be 
filtered rapidly by the use of suitable apparatus, as illustrated in Fig. Io. At the connection with the vacuum pump a valve should be inserted-or a flask with a rub-

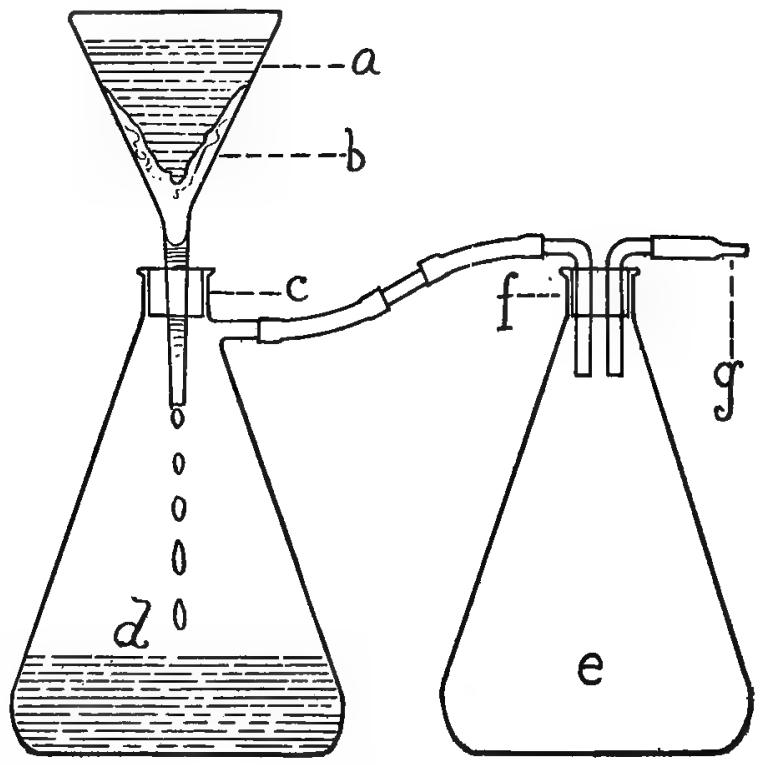

FIG. I0

Filtering Media by Means of Vacuum Pump
$a$. Liquid medium
$b$. Absorbent cotton
c. Rubber stopper
d. Filtered medium

c. Reflex flask

f. Rubber stopper with two holes

g. Connection with aspirator

ber cork with two holes may take its place-to prevent the water from entering the flask.

Whichever method is employed, the filter must always first be soaked in hot water, or, better, kept in steam until used. 


The filtered liquid is collected in a smaller funnel (Fig. 5, c). A wire basket should be supported so as to be inclined to an angle of about $45^{\circ}$, so as to allow the proper arrangement of the tubes.

Remove the plug of a culture-tube and quickly pass the latter up the full length of the pipette (Fig. 5, f), which should be no less than 3 inches long. Open the pinchcock, fill the tube one-third full, and then quickly withdraw the tube so as to leave no trace of the medium on the upper part of the tube, and replace the cotton stopper. If this precaution is neglected, the cotton stopper will stick to the glass, which will cause a great deal of annoyance later on and $\mathrm{ex}$ pose the medium to the danger of contamination. Fill thirty tubes in this manner.

If an exact volume of medium is required in a test-tube, an apparatus such as is shown in Fig. II may be employed.

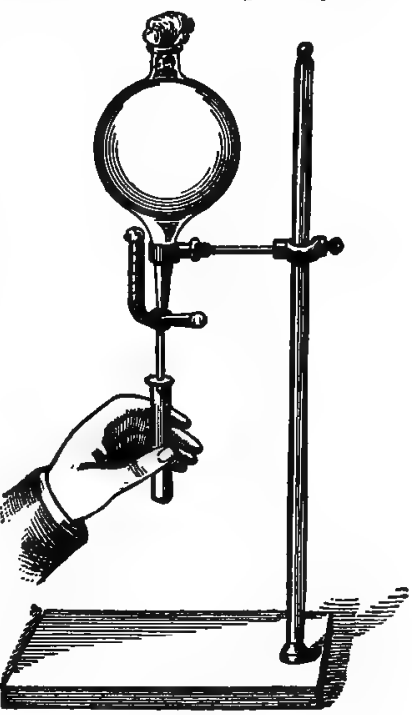

FIG. II

Filling Definite Amount of Medium into Culture-Tube

EXERCISE II. PREPARATION OF GLUCOSE-AGAR

Glucose-agar is especially used for the demonstration of gas-forming organisms. In such cases the glucose is decomposed, and gas appears in bubbles throughout the medium. 


\section{Preparation-}

I. Note the weight of an Erlenmeyer flask ( $500 \mathrm{cc}$ c).

2. Weigh 3.75 ( $1 \frac{1}{2}$ per cent.) g. of glucose into the flask.

3. Filter $25^{\circ} \mathrm{g}$. of plain agar into the flask, heat, and agitate until the glucose is completely dissolved.

4. Fill twenty tubes in the same manner as above.

NoтE.-By tying a string across a wire basket near the top, the plain agar-tubes may be kept separate from the glucose-agar. A slip of paper indicating the medium and the date of its preparation must always be inserted. These two media are very diffcult to distinguish by the eye, and failure to label them properly will lead to entirely unreliable results.

The media should now be sterilized in the autoclav. The principle of this mode of sterilization is the application of steam under pressure. Certain bacteria, and some of these are very widely distributed in nature, have the faculty of forming spores. These spores are very highly resistant to heat and do not lose their vitality either by boiling or by application of heat under ordinary atmospheric pressure, although all vegetative forms are killed. By adding the pressure of one atmosphere to ordinary pressure, the boiling-point is raised to $121.40^{\circ}$, which is sufficient to kill all spores during an exposure of 5 minutes; media in flasks should be given ro minutes.

The autoclav consists of a strong cylinder, made of iron, with a bottom and removable lid. Inside is a basket, or rack with shelves, resting on three short supports with a centrally located hole in the bottom. The lid fits closely (in some autoclavs with a large washer), and is provided with a steam-valve (Fig. I2, a), a safetyvalve (Fig. $\mathbf{1} 2, b$ ), and a gauge (Fig. I2, c). The latter indicates the pressure and temperature. The top is 


fastened by means of thumbscrews (Fig. I $2, d-d$ ). The heat is produced by two circular sets of Bunsen burners in a hollow cylinder at the bottom (Fig. I2,e).

Other forms based on the same principles are also in use.

Before using the autoclav the inside should be exam-

FIG. I2

Autoclav

4. Steam-valve

b. Safety-valve

c. Gauge

$d-d$. Thumbscrews

e. Bunsen burner and opening

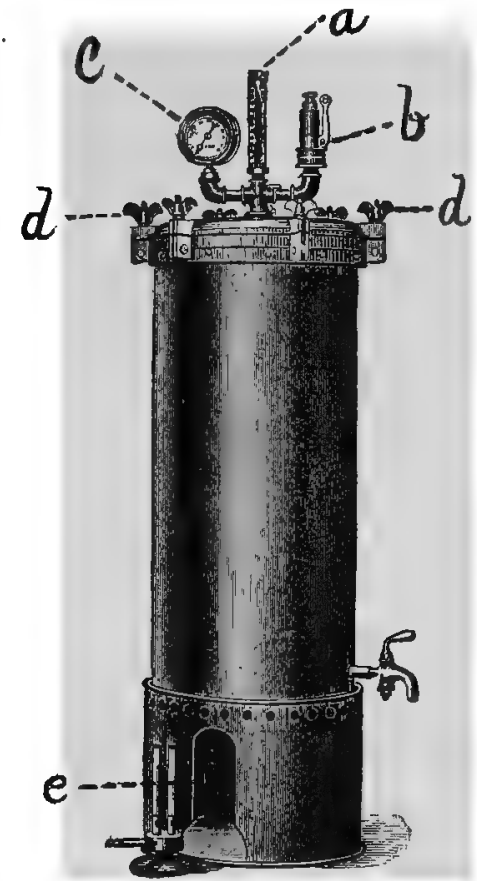

ined. It must be clean, and there must be enough clean water in it to reach nearly to the bottom of the basket. Water containing large amounts of impurities is liable to foam when boiling, and thus wet the plugs and ruin the 
medium. Place the material to be sterilized in the basket. Fit the lid of the autoclav by joining corresponding marks (usually a number or a letter) on the side of the lid and margin of the main body. Then tighten the thumbscrews by hand, always tightening those diametrically opposite to each other at the same time. See that the steam-valve is open, and then light the gas. Regulate the safety-valve to blow off at the required pressure, if provided with the means of doing this.

It is necessary to allow the steam to escape for about one minute, in order to drive all air out of the cylinder. Then close the steam-valve. The pressure will begin to increase, as indicated by the hand of the manometer. When the desired temperature is reached, shut off the gas from the outer ring of burners and regulate the gas pressure of the inner rings, to maintain the proper temperature. It is sufficient to expose the media to a temperature of $\mathrm{I} 20^{\circ}$ for 5 minutes. After this has been accomplished, shut off the gas and allow to cool to normal pressure, or $100^{\circ}$ or below. The lid should not be released before this, as a sudden diminution of pressure causes the contents of the tubes suddenly to boil up and push the stoppers out.

The steam-valve should be opened when the autoclav has cooled to $100^{\circ}$ or below. This will guard against the possibility of the top blowing off and injuring the operator, if the gauge does not register properly. On the other hand, the suction caused by the contraction of the steam may hold the lid down, so that it cannot be removed until the pressure is equalized.

When sterilizing media which contain carbohydrates (glucose-agar, for instance), it is important not to allow 


the temperature to rise beyond $120^{\circ}$, nor to allow it to remain at that point any longer than 5 minutes. Carbohydrates are easily broken up by heat. They are then valueless in the medium, and also generate an acid reaction as a result of chemical decomposition.

In the preparation of agar more difficulties are met with than in the preparation of any other medium. The main difficulty is the fact that it takes a long time-from 30 to 45 minutes-for the agar to dissolve, and this creates the danger of burning it by overheating. Great care must therefore be exercised in the preparation at all stages.

EXERCISE III. PREPARATION OF PEPTONE GELATIN

I. Weigh the saucepan and measure 350 c.c. of tap-water into it. This amount includes 50 c.c. allowance for evaporation.

2. Dissolve $0.75 \mathrm{~g}$. of extract of meat, and, when near boiling, add $3 \mathrm{~g}$. of peptone.

3. When boiling, add during the cold season ro per cent. ( $3 \circ \mathrm{g}$.), during the warm season 12 per cent. ( $36 \mathrm{~g}$.), of the best gelatin (Gold label) by dissolving two or three leaves at a time, with constant stirring.

4. When completely dissolved, adjust the reaction as directed in the preparation of agar. As gelatin contains considerable acid, it will take more $\mathrm{NaOH}$ solution in proportion than for agar.

5. Then cool to $60^{\circ}$, and stir into the mixture the white of one egg dissolved in 30 c.c. of water. Slowly heat over the flame on a piece of asbestos, without stirring, until the egg albumen is completely coagulated and forms a dry film on top. 


\section{LABORATORY GUIDE IN BACTERIOLOGY}

6. Adjust the weight, and filter through paper or absorbent cotton previously moistened with hot water, and distribute into thirty culture-tubes.

7. Sterilize in the autoclav for 5 minutes at $120^{\circ}$. Care is necessary not to overheat this medium, because gelatin easily loses the property of solidifying when cool. In order to avoid this, the discontinuous or intermittent method of sterilization is often used. By this method the medium is exposed in a steam sterilizer to steam at the temperature of $100^{\circ}$ for 20 minutes on three consecutive days. The first day all vegetative forms are

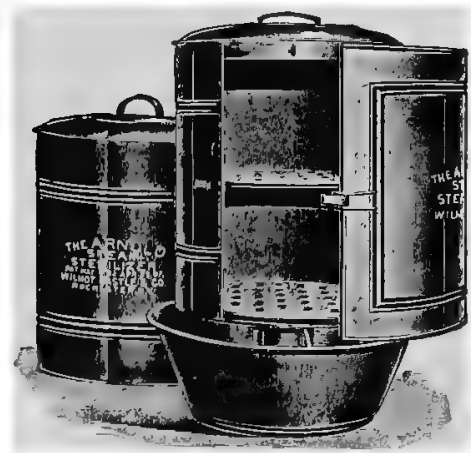

FIG. 13

Arnold Steam Sterilizer

The hood is taken off and the door opened, showing inside arrangement

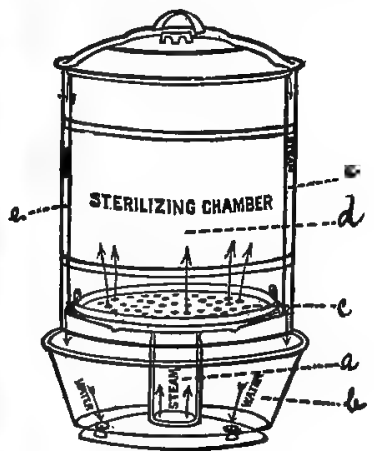

Fic. 14

Arnold Steam Sterilizer

a. Inner water compartment

b. Outer water compartment

c. Perforated bottom

d. Sterilizing chamber

๘. Sheet-copper walls

killed. By keeping the medium at room or incubator temperature, spores, which may be present, will develop into the vegetative form and be killed by the second day's exposure. If after this any spores should happen to 


survive, they will develop during the next 24 hours, and the third exposure to steam will complete the sterilization. The apparatus used for this purpose is the "Arnold Steam Sterilizer." The usual form used is seen in Figs. $\mathrm{I} 3$ and I4. Fig. I3 illustrates the appearance of the ordinary form with the hood off. Fig. I4 shows the inside arrangement, $a$ and $b$ being two compartments connected by small holes, and in which a certain amount of water has to be kept. The water contained in the inner compartment $(a)$ is brought to a boil by a Bunsen burner, the steam rising through a number of holes in the bottom (c) into the chamber $(d)$. The steam condenses at the top of the chamber and returns between two sheet-copper walls $(e, e)$ to the large compartment $(b)$. A larger form of steam sterilizer, constructed on the same principle, is convenient for sterilizing large amounts of the media.

EXERCISE IV. PREPARATION OF PEPTONE-BROTH (FRENCH: BOUILLON) AND DUNHAM'S PEPTONE-SOLUTION

I. Weigh the saucepan and measure 600 c.c. of tapwater into it and heat.

2. Dissolve, when hot (but not boiling), $6 \mathrm{~g}$. of peptone.

3. When completely dissolved, replace the evaporated water and divide into two equal amounts (300 c.c. each).

4. Filter one part twice through the same filter (paper) and distribute into twenty-five tubes. Sterilize in autoclav for 5 minutes at $120^{\circ}$. This is Dunham's peptone-solution.

5. Dissolve completely I $g$. of extract of beef in the other 300 c.c. 


\section{LABORATORY GUIDE IN BACTERIOLOGY}

6. Adjust the reaction in the same manner as above.

7. Fill into an Erlenmeyer flask and autoclav. for Io minutes at $120^{\circ}$

8. Keep the Erlenmeyer flask and contents for 24 hours in a cool place, filter, and then distribute into

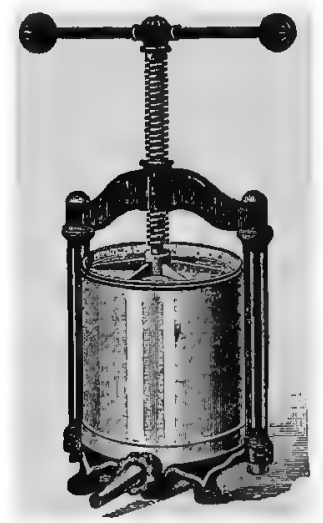

FIG. I5

Meat-Press

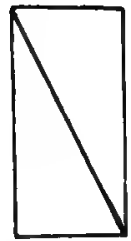

F IG. 16

Potato Cylinder showing diagonal for cutting

twenty-five tubes, and autoclav these at $120^{\circ}$ for $5 \mathrm{~min}$ utes. This is "peptone-broth."

NoTE.-The reason for exposing broth to a heat of $120^{\circ}$ twice is this: The solution contains a considerable quantity of substances, which are precipitated by heat and appear as a sediment after cooling. As it is important to have a perfectly clear broth in tubes, these substances are precipitated by the first heating, and, if tubed later, the second sterilization will not affect the appearance of the medium.

Broth was originally prepared from chopped beef, and for many purposes this is preferable. The method of preparation is as follows: 


I. $500 \mathrm{~g}$. (I pound) of lean minced beef, as free as possible from fat and tissues, is added to rooo c.c. of tap-water and set aside on ice for 24 hours.

2. Weigh a saucepan, and cook the beef and water for about one hour.

3. Strain through cheese-cloth, and press all the liquid out in a meat-press (Fig. I5).

4. Replace the water lost by evaporation.

5. Dissolve Io g. of Witte's peptone.

6. Adjust the reaction.

7. Filter into flasks, and sterilize in autoclav at $120^{\circ}$ for to minutes. It may then be distributed into culturetubes.

Fresh meat, as well as extract of meat, often contains a small amount of muscle-sugar (glycogen). If it is necessary to prepare a sugar-free broth, a pure culture of Bucillus coli is added to the mixture of chopped beef and water, and this is incubated at $37^{\circ}$ for 24 hours. After this the preparation is the same as described.

\section{EXERCISE V. PREPARATION OF POTATO}

I. Select several large potatoes, and cleanse by brushing all dirt off carefully and washing in water.

2. Punch out cylinders with a borer of suitable size, trim them, and cut them into two equal parts (Fig. I6). Cut with a sharp knife along the diagonal.

3. Immerse the pieces in running water for 24 hours.

4. Insert one half-cylinder into each potato-tube so that the wide end rests on the constriction, then pour a small amount of water into it. If the potato-tubes have no constriction, place a small wad of cotton in the bottom and moisten this with water (Figs. 17 and 18 ). 
5. Sterilize in autoclav for 5 minutes at $120^{\circ}$, or in Arnold for 3 consecutive days with 20-30 minutes' exposure.

EXERCISE VI. PREPARATION OF LITMUS MILK

I. Separate five-sixths of the cream from the milk.

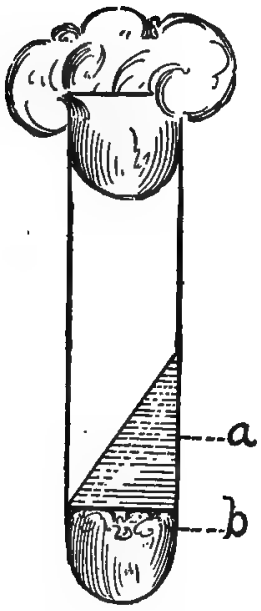

Fig. I7

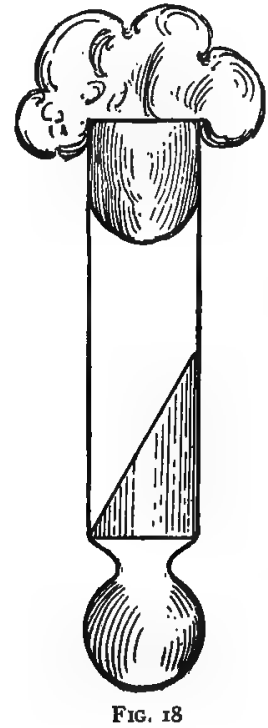

Potato-Tube

Ordinary Style of Potato-Tube w. Potato b. Cotton

2. Add 7 -Io per cent. of a solution of litmus (tincture of litmus).

3. Distribute about 7 c.c. into each of twenty-five tubes.

4. Sterilize in autoclav for .5 minutes at $120^{\circ}$, or in Arnold for 3 consecutive days.

NoTE.-The prepared culture media should be carefully stored in a dark, cool place. If they are to be kept for a consid- 


erable length of time, they should be sealed either with paraffin or with a rubber cap. They should always be protected from dampness, as mold fungi are apt to alight on the cotton stoppers and send their filaments into the tube. Before inoculation, they should always be carefully examined, and those which show cloudiness or colonies, as well as those which have shrunk from evaporation, should be rejected. 


\section{CHAPTER IV \\ PREPARATION OF STAINING SOLUTIONS}

Saturated alcoholic solutions of stains are prepared by covering an arbitrary amount of stain with absolute alcohol. The solution is saturated as long as any of the stain remains undissolved at the bottom of the vessel. Other solutions necessary for the preparation of stains are:

I. Solution of potassium hydrate in water $I: 10,000$.

2. Solution of carbolic acid in water (5 per cent.).

3. Anilin-water, prepared by shaking anilin-oil with water ( 2 per cent.) and filtering twice through the same paper. It should be perfectly clear.

The staining-bottles usually employed have a capacity of about 30 c.c. The following amounts will nearly fill them:

I. Loeffler's methylene-blue: Saturated alcoholic solution of methylene-blue g c.c. Potassium hydrate in distilled water I: 10,000 2 I c.c.

2. Ziehl-Neelsen's carbol-fuchsin:

Saturated alcoholic solution of fuchsin..... 3 c.c. $5 \%$ carbolic acid solution in water........27 c.c.

3. Ehrlich's anilin-gentian-violet:

Saturated alcoholic solution of gentian-violet 7.5 c.c. Anilin-water.................... 22.5 c.c.

This stain requires occasional filtering and is somewhat unstable.

4. Gram's iodin solution:

Iodin...........................

Potassium iodid...................... $0.2 \mathrm{~g}$.

Dissolve in about 2 c.c. of water, and then add enough water to make the total measure 30 c.c. 




\section{CHAPTER V}

\section{THE MICROSCOPE}

(Fig. I9)

The compound microscope is a necessary adjunct to any kind of bacteriological work. For this work three objectives (Leitz No. 3 , No. 6, or No. 7 and ${ }_{1}^{\frac{1}{2}}$ oil immersion, Zeiss No. A or No. AA, No. D or No. DD, and $\frac{1}{12}$ oil immersion) and two oculars (Nos. 2 and 4 ) are indispensable. For the intelligent manipulation of the microscope it is absolutely necessary to understand the underlying optical principles, which may be studied from special works on the subject.

\section{Rejerences-}

S. H. Gage, The Microscope.

Carpenter and Dallinger, The Microscope and its Revelations.

For use in the laboratory it will be sufficient to call attention to some of the most important points to be observed.

The usual pattern of microscope consists of two main parts: the stand, and the optical parts (Fig. I9) which are attached to the stand.

The stand consists of a body-tube, draw-tube, coarse adjustment, fine adjustment (micrometer screw) in a pillar, nose-piece, stage with clips for holding the object, main pillar, and the horseshoe base. At the junction of the main pillar and the fine-adjustment pillar is the inclination joint.

The draw-tube, regulating the focal length, which varies in different instruments, should be raised to I 6 


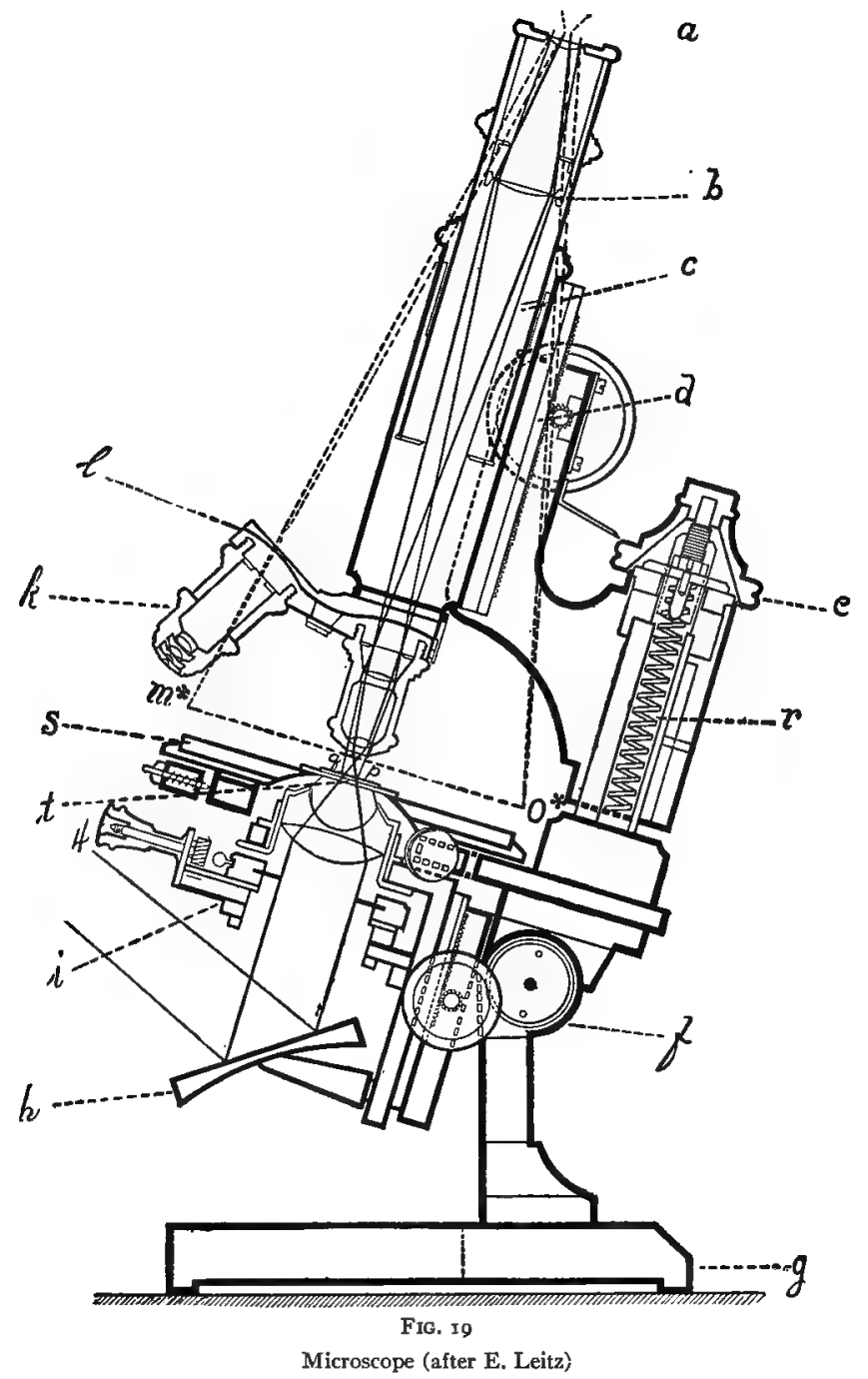
a. Ocular b. Place where virtual picture is
formed
i. Iris Diaphragm and Condenser
z. Body-tube
$d$. Coarse adjustment
e. Micrometer screw
$f$. Inclination joint
g. Horseshoe base
$k$. Objective
l. Nose-piece
$m-o$. Picture as it appears to the eye p.-g. Object (cover-slip)
$r$. Adjustment spring
s. Stage
t. Object (slide)
h. Mirror 


or $17 \mathrm{~mm}$. If a nose-piece is attached, the width of this must be deducted from the tube-length.

The optical parts are the oculars, the objectives, the substage condenser, and the mirror. The ocular is a combination of lenses, which slips into the top of the draw-tube and is nearest the eye. The objective is a combination of lenses which is screwed into the nosepiece and fits to the lower end of the draw-tube. The substage condenser fits under the stage. It concentrates the light on the object and is raised for high powers or lowered for low powers. At the lower end of this condenser is the iris diaphragm, which is regulated by a small lever with a milled head, and serves the purpose of regulating the light supply. The mirror has two sides, a concave and a flat.

In the manipulation of the compound microscope the following points should be strictly observed:

I. Keep the instrument scrupulously clean. When not in use, lock it in the case or cover it with a bell-jar.

2. When carrying the instrument, grasp it by the main pillar underneath the stage, not by the fine-adjustment pillar. The fine adjustment consists of a very delicate screw-thread, which is easily damaged.

3. The lenses, condenser, and mirror, when needing, should be wiped with Japanese lens-paper, never with any coarse material.

4. For cleaning use a damp cloth. For wiping the lenses use water or xylol. Never use alcohol, as this dissolves the cement holding the lenses in place and also injures the lacquer.

5. Do not take the instrument apart. The working parts are of extremely delicate nature and easily injured. 
Be careful not to drop the oculars or objectives.

6. The inclination joint can be used only with dry lenses and dry objects, not with the oil-immersion or hanging-drop preparations.

7. After placing the object on the stage, focus with a low power and the coarse adjustment. With high powers use the coarse adjustment first and the fine adjustment afterward. The free use of the fine adjustment saves the accommodation of the eye. As the eye is capable of accommodating itself to distances, it may with an effort distinguish a picture which is not in perfect focus. This effort is saved by using the fine adjustment.

8. Always raise the draw-tube by means of the coarse adjustment before changing the objectives or examining a different preparation.

9. Before focusing, obtain as good a light as possible by turning the mirror, and then regulate the supply by the diaphragm. Always use reflected light, never direct sunlight.

I0. When focusing an object, lower the draw-tube until the lens almost touches the cover-glass. This can easily be seen by looking at the instrument from one side and watching the reflection of the objective in the coverglass. Then, with the eye at the ocular, slowly focus up. Do not focus down with the eye at the ocular, as the lens may then come into violent contact with the object, destroying the latter and injuring the lens.

II. For living or transparent objects use as little light as possible. For stained or opaque objects more light is necessary.

12. Do not use higher powers than is necessary.

13. To use the oil-immersion lens, place a drop of 


clear cedar oil, free from dust and air-bubbles, on the cover-glass, which must be perfectly dry. In this case, by careful manipulation, the objective, after being brought in touch with the oil by means of the coarse adjustment, may be gradually lowered by the fine adjustment until the object is focused; or lower the objective until almost in touch with the cover-glass, and focus up. High powers always require the use of a homogeneous liquid between the cover-glass and the front lens of the objective, to avoid loss of light by refraction. As a bundle of rays disperses when cntering a thinner medium from a denser one, there is not sufficient light entering the objective to make objects discernible, when using high powers. By the insertion of a liquid (inspissated oil of cedar) of the same, or nearly the same, refractive index, a homogeneous connection is established between the cover-glass and the objective, thus avoiding this loss and allowing a bundle of light of sufficient power to enter the objective. ${ }^{\mathrm{I}}$

I4. After using the oil-immersion lens, wipe the oil off with lens-paper. If the oil sticks to the lens, wipe it off with xylol, never with alcohol. At the same time wipe the oil off the cover-slip.

15. The microscope should stand on a firm table, to avoid being shaken. The table should be low enough so as not to necessitate bending the body.

16. Always keep both eyes open. This saves the eyesight. Beginners find this a difficult rule to apply, but with very little practice and persistence it is easily accomplished. Also use both eyes alternately.

I7. It is always well to move the object while bringing

For detailed description and diagrams see S. H. Gage The Microscope. 


\section{LABORATORY GUIDE IN BACTERIOLOGY}

it into focus. It is then easy to feel if the lens touches the glass, and a moving object is seen much more easily than a stationary one.

18. Use the plain mirror in combination with the condenser, and the concave mirror without the condenser or with artificial light.

I9. In preparing stained preparations, it often happens that a small amount of stain remains on the upper side of the cover-slip. Care must be taken to focus for a plane below this. 




\section{CHAPTER VI}

COLLECTING BACTERIA FROM THE AIR

I. Sterilize all Petri dishes in the hot-air sterilizer for one hour at $160^{\circ}$

2. Melt two tubes of plain agar and one of glucoseagar in the water-bath. The water-bath (Fig. 20) is a round copper vessel with a number of holes in the top.

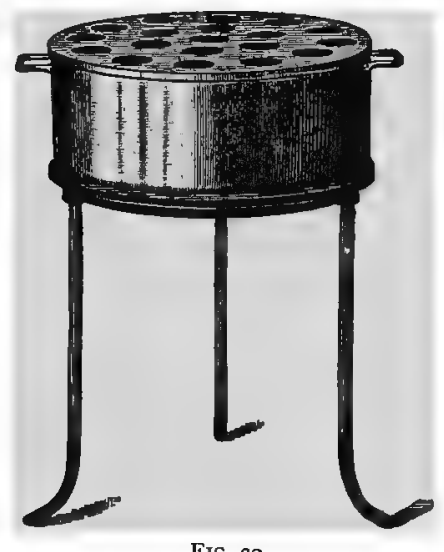

FIG. 20

Water-Bath

These holes are large enough to allow a culture-tube to slip in easily. A thermometer is passed through a rubber cork with a hole in its center, and inserted into one of the holes in the water-bath. Enough water is then poured into the apparatus to fill it about two-thirds, and the thermometer is lowered until the mercury bulb is 


\section{$3^{8}$ LABORATORY GUIDE IN BACTERIOLOGY}

completely immersed in the water. The culture-tubes are then slipped in, and the water is heated to roo?.

3. Singe the cotton stopper of the liquefied agartubes in the flame, remove the cotton stopper, pass the mouth and about one inch of the tube through the flame, and pour the contents into a sterile Petri dish, carefully lifting the cover (Fig. 2I) and quickly replacing it.

4. Repeat this operation with the second agar-tube.

5. Place both Petri dishes containing the liquid agar on a level surface.

6. When the agar is solidified, expose, by removing the cover, one dish to the air of the laboratory, and the other outside on the window-sill, for ro minutes.

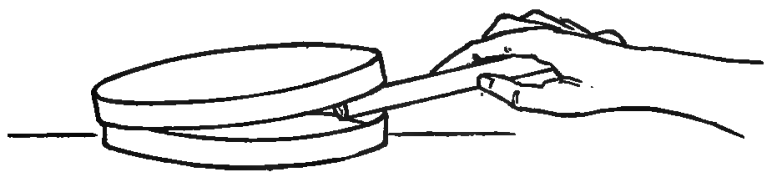

FIG. 2I

Pouring Medium into Petri Dish

7. Replace the cover and place in lockers.

8. Cool the water-bath to $43^{\circ}$ exactly, and mix the scrapings from under a finger nail with the liquid glucoseagar. Keep this also in the locker.

9. Remove the plug of a tube of broth and place a hair in the liquid. Keep also in the locker.

When inoculating liquefied agar media, it is necessary to do so at a temperature no higher than $43^{\circ}$ nor lower than $40^{\circ}$. Above $43^{\circ}$ the organisms are liable to be injured by heat; below $40^{\circ}$ the agar solidifies, and an even distribution is impossible. If gelatin is used, the latter precaution is not imperative, as gelatin solidifies at 


about $25^{\circ}$. Observe and make notes on the appearance of these Petri dishes after 24 hours. By this time it will be observed that a number of spots of different sizes, shapes, colors, etc., have formed on the surface of the medium. Each of these spots probably consists of a large number of the same species of organism, and is called a colony. Besides bacteria, yeasts and mold spores are constantly present in the atmosphere and drop on the plate. These organisms, being of higher specific gravity than the air, are constantly falling, or are carried hither and thither by currents in the atmosphere. Small particles of dust also may alight on the medium, and then form a dark spot near the center of the colony. Dust particles are usually covered with bacteria, possibly of different species, and in this case the resulting colony may not be composed of one and the same species. These are not "pure cultures."

Inoculate two or three colonies on agar-slants.

Note.-Agar-slants are prepared by allowing liquid agar to solidify in a slanting position.

\section{Method of inoculation-}

I. Singe the cotton plug of a tube containing the medium. It is imperative always to singe the cotton plugs of tubes before opening them, whether these tubes contain a culture or a sterile medium. The organisms from the air are constantly falling on the cotton, and bacteria may also be deposited on the cotton by handling it with the fingers. If these organisms were not killed by the process of singeing, they would drop on the medium after removal of the stopper, and thus ruin a pure culture.

2. Hold the tube (or, if a transfer is made, both tubes side by side) between the thumb and the forefinger, so 
that the end of the tube rests on one edge of the hand (Fig. 22), holding them at an angle of about $45^{\circ}$. If held horizontally, the condensation water, always present at the lower end of the agar medium, will moisten the surface and destroy a characteristic growth along the needle-track. If held in a vertical position, contamination from the air cannot be avoided.

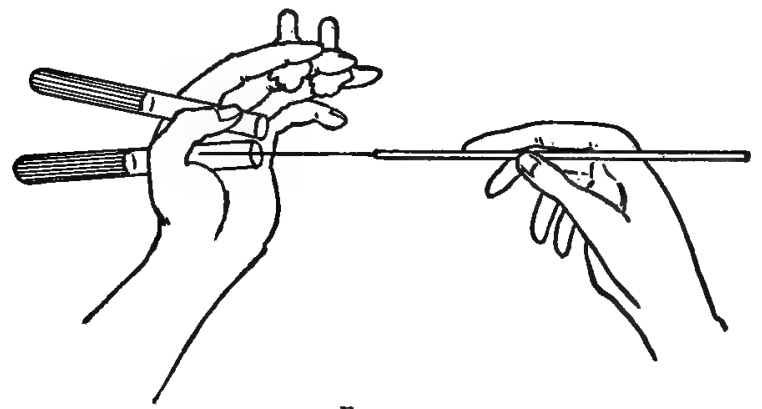

FIg. 22

Method of Inoculating Media

3. Remove the cotton stoppers by taking hold of the singed portion only, and hold also by the singed part only between the other fingers. If the portion of cotton from the inside of the tube is touched by the fingers, or accidentally falls on the table or the floor, it becomes contaminated and must be singed before replacing.

4. Sterilize the straight platinum needle in the flame, holding it like a pencil. The platinum wire should be heated until red-hot, and the glass end passed slowly through the flame once or twice.

5. After cooling the needle by plunging into the medium, take up a small portion of a colony or culture by a lateral movement on the end of the needle, and, 


when removing it from the tube, take care not to touch the walls of the tube. If any cotton accidentally sticks to the mouth of the tube, it should be burnt off with a hot platinum needle, and then the mouth of the tube passed through the flame before inserting the needle.

6. Insert the needle with the culture into the sterile tube, and carefully draw along the surface of the slant, turning the needle during the operation and without puncturing the jelly.

7. Sterilize the needle in the flame, as above, and replace the cotton plugs. It is not superfluous to repeat here that the platinum needle should always be flamed immediately after taking it up, and again before laying it down.

A stab-culture is made in the same manner as a slantculture, except that the medium is punctured centrally by a quick, steady movement of the needle. Care should be taken not to let the needle touch the bottom of the tube, the object being to make as narrow a puncture as possible, and by touching the glass the needle would bend and make a ragged opening in the medium on withdrawing.

Inoculations of liquid media are made the same as agar-slants, except that the end of the needle is gently rubbed against the glass below the surface of the liquid. The medium is then shaken. After inoculation milkcultures should never be shaken again, as this might destroy a characteristic shape of the coagulum or break up the cream-ring.

Inoculations from liquid media are made with the looped needle. This may also be used, if a considerable amount of growth is required on a solid medium. The 
contents of the loop are then spread over the whole surface.

Describe the appearance of the colonies as to-

r. Naked-eye appearance:

Color.

Shape.

Border.

Size (approximately in millimeters).

2. Appearance under low power of microscope.

3. Give the approximate number of colonies on the plate.

4. Examine as to motility in the hanging-drop. Preparation of a hanging-drop (Fig. 23):
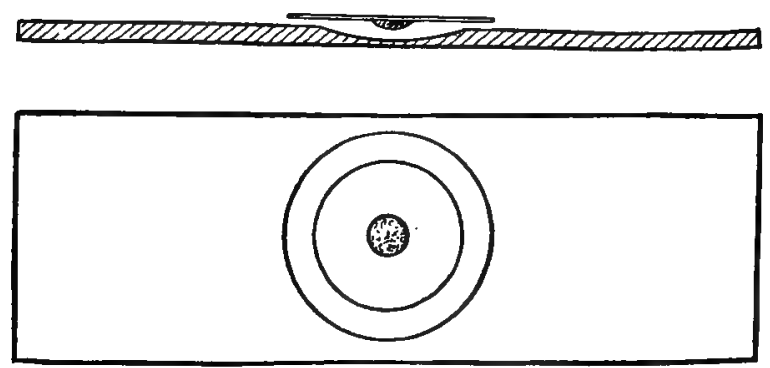

FIG. 23

Hanging-Drop

(After Muir and Ritchie)

I. Clean a cover-slip in alcohol. Pass several times through the flame so as to burn the last traces of grease off the surface.

2. Place a loopful of pure water on the center of the cover-slip.

3. Flame the straight platinum needle, and, after 


cooling, touch one of the colonies and mix lightly with the drop of water without spreading it. Take only a very minute amount of culture, so as to produce the faintest visible cloudiness in the water.

4. Smear vaselin around the depression in a hollowground slide, quickly invert the cover-slip over the depression, and gently press the margin on the vaselin.

5. Examine in oil, using very little light.

Brownian movement.-Rub a small amount of carmine in a mortar with some water and make a hanging-drop preparation. When examining this through the oilimmersion lens, it will be observed that the small particles of carmine have a lively vibrating motion. This is called "Brownian" or "molecular movement," or "pedesis." The particles scarcely change their relative position. Actively motile organisms, on the contrary, widely change their relative positions. The movement of these may be slow, snake-like, or like a fish swimming; or they may dart rapidly across the field.

Now observe and describe what changes have taken place in the tube of broth with the hair. Compare with a sterile tube, noting the turbidity, sediment, odor, etc. Also examine in hanging-drop.

Examine the tube of glucose-agar containing the nailscrapings. Describe the general appearance, and note whether gas-bubbles are present.

Finally, from the stained preparations make sketches of the appearance of the culture medium, the shape of the colonies, and the morphology of the organisms.

Make stained preparations of three different colonies, using the three stains; i. e., gentian-violet, methyleneblue, and carbol-fuchsin. 
Method of making stained preparations-

I. Clean and flame a cover-slip, or, if preferred, a slide may be used for this purpose. Cover-slips, if handled by the fingers, should be held by the edges. Use as much as possible the forceps made for that purpose. After handling the forceps they should be sterilized in the flame.

2. Place one loopful of water on the cover-slip.

3. Take the minutest quantity of the colony or culture on a platinum needle and mix gently with water until faintly cloudy. Burn the remainder of the culture off the needle.

4. Spread over the cover-slip by two or three sweeps of the needle. The water should spread easily and not run together. If this takes place, the cover-slip has not been cleaned sufficiently.

5. Dry by moving high over the flame.

6. Pass three times back and forth through the flame rapidly. This process precipitates albuminous matter and causes the bacteria to adhere firmly to the glass.

7. Cover with stain for Io-I5 seconds.

8. Wash in water and examine. If satisfactory:

9. Float the cover-slip off the slide with water. Blot with filter-paper, dry in the air or high over the flame, and mount in Canada balsam.

Io. Label and preserve this preparation.

Try to avoid the mistake, made by most beginners, of taking too much growth on the needle. For hangingdrop preparations less material should be used than for a stained preparation. 




\section{CHAPTER VII}

\section{EXERCISES ON INFECTION AND STERILIZATION}

EXERCISE I. PHENOMENA OF INFECTION

I. Prepare three agar-plates as directed in Chap. VI.

2. Touch the surface of the jelly in one plate with the tips of the fingers.

3. Touch the surface of the jelly of another plate with the tips of the fingers, after washing the hands carefully.

4. Catch a fly and allow it to walk on the surface of the jelly of the third plate for a few seconds. Release the fly and replace the cover.

5. Place these three plates in a locker or thermostat for 24 hours. Observe and describe the results. Make hanging-drop and stained preparations of some of the colonies formed.

EXERCISE II. PHENOMENA OF STERILIZATION

I. Make an infusion of hay in a flask with cotton stopper. (See Appendix I.)

2. Set the flask aside for 24 hours in a warm place, and observe the results. To what are these results due?

3. Expose a tube of unsterilized broth to steam in the "Arnold," another to steam in the autoclav, at $120^{\circ}$ for 5 minutes each.

4. Set aside in a thermostat, and observe the results.

EXERCISE III. PHENOMENA OF STERILIZATION (CONTINUED)

Action of Berkefeld and cotton filters.-Berkefeld filters are made of diatomaceous earth, and are porous so as 


\section{LABORATORY GUIDE IN BACTERIOLOGY}

to allow liquids to pass through, but no solids or organisms.

r. Arrange a Berkefeld filter so as to connect with the suction pump, and filter a quantity of unsterilized broth (Fig. 24).

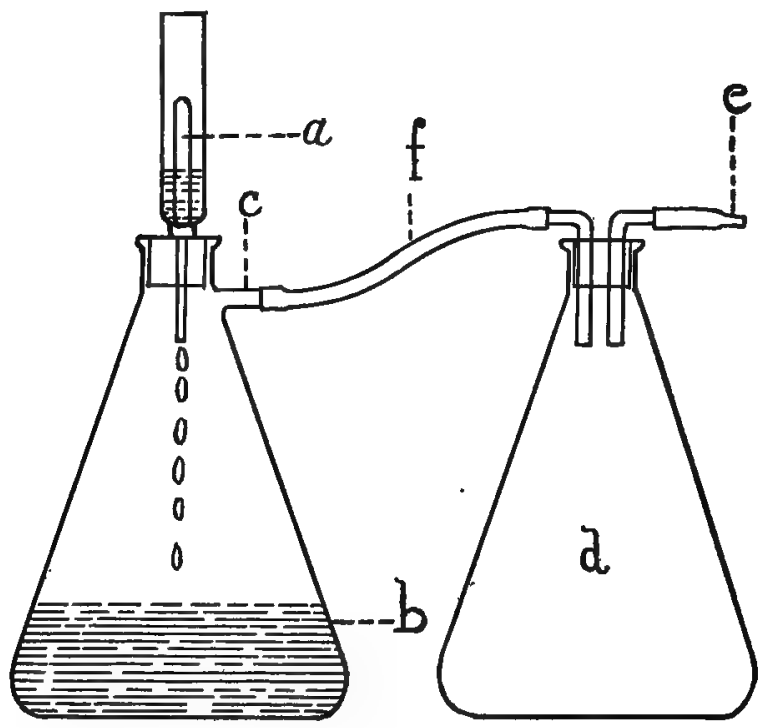

FIG. 24

Action of Berkefeld Filter

a. Berkefeld filter

b. Filtered liquid

c. Side tube with cotton filter d. Intercepting flask

- e. Connection with aspirator

f. Rubber hose

2. Set aside, and observe the results. The filter (a), after being connected with the flask, is sterilized in the autoclav. The cotton plug at $c$ prevents the air, which is sucked back, from carrying germs. The flask $d$ will 


prevent the water from running back and mixing with the filtered liquid.

Now arrange a cotton filter as shown in Fig. 25. Vessel $a$, provided with a rubber stopper $(b)$ with two openings, is arranged so as to have a glass tube (c) reach to the bottom. This tube is provided with cotton at

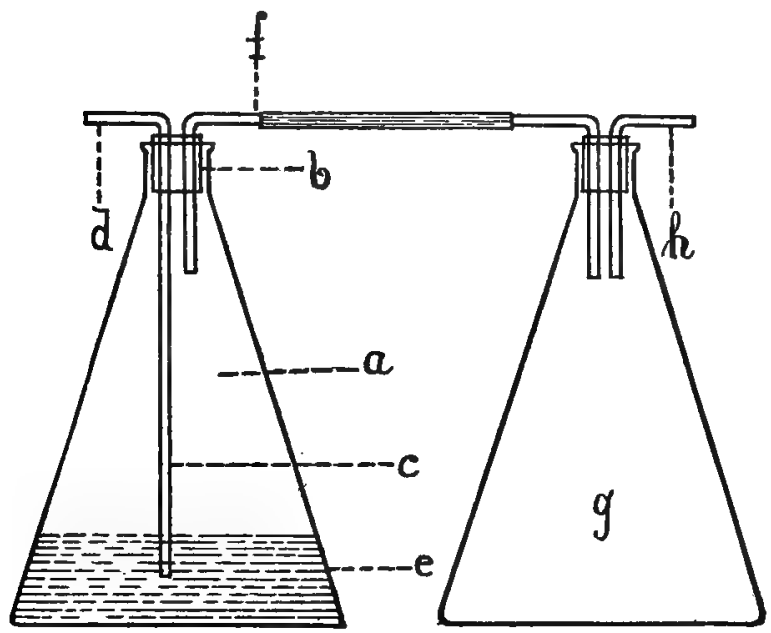

FIG. 25

Action of Cotton Filter

a. Erlenmeyer flask

b. Rubber stopper

c. Glass tube

d. Cotton filter

e. Broth f. Bent glass tube with cotton filter at $\mathbf{f}$.

g. Erlenmeyer flask

$h$. Tube connecting with aspirator

the top $(d)$ and some nutritive medium (broth, $e$ ) is placed inside. Through the opening a bent glass tube leads out, and this tube is also provided with a cotton filter at $f$. The whole apparatus is then sterilized in 


\section{LABORATORY GUIDE IN BACTERIOLOGY}

the autoclav at $120^{\circ}$ for 5 minutes, and connection is made through the flask $(g)$ and the tube $(h)$ with the aspirator. Now aspirate some air through the flask, disconnect at $f$, and set aside. Observe the results. Why does no growth take place? Remove the cotton filter (d), and drop it into a flask containing sterile broth; place in the thermostat for I8-24 hours; note and examine the conditions then present. 




\section{CHAPTER VIII}

\section{STUDY OF YEASTS, MOLDS, AND TORULE}

EXERCISE I. CULTURAL STUDIES

Read carefully the chapter on yeasts and molds in the textbook. Yeasts, torulæ, and molds grow better in a medium of a slightly acid reaction than in a neutral or alkaline medium. Wort-agar or gelatin prepared as described (Appendix I) will answer very well for this purpose.

I. Melt eight wort-agar tubes in the water-bath.

2. Pour the contents of two tubes into sterile Petri dishes, and set aside to solidify.

3. Allow the other six tubes to solidify so as to form a slanted surface.

4. After the plates have cooled, expose them to the air in two different places for Io or I5 minutes.

5. Place in your locker.

6. Examine after 24 or 48 hours. Describe what you see.

7. Transfer several mold and yeast colonies to the slants prepared.

8. Make transfer of a stock-culture of Saccharomyces cerevisiae, or any other species of yeast.

NotE.-Molds may easily be recognized by the filamentous, cotton-like form of the colonies. The hyphæ extending into the air carry the spores (conidia). By gently touching these with a sterile platinum needle, the spores may be transferred to the agar-slant, and development will take place after 24 hours. Colonies of yeasts or torulæ appear smooth, moist, opaque, elevated, and slightly yellowish-white, or sometimes reddish. 
These may be transferred in the same manner as colonies of bacteria. Molds require very careful handling for microscopical demonstration. They are best examined in water in the unstained condition.

Method of preparing molds for microscopical examination-

I. Transfer some of the growth to alcohol (5० per cent.).

2. When thoroughly moistened, transfer some of the growth to a drop of glycerin on a slide.

3. Spread carefully with a platinum needle.

4. Cover with a slip and examine.

5. If satisfactory, the preparation may be made permanent by painting a ring of asphalt around the edge of the cover-slip.

Molds may also be stained in the following manner:

r. Place a small amount of mold on a slide.

2. Cover with alcohol.

3. Wash in water.

4. Stain with gentian-violet or methylene-blue.

5. Mount in glycerin.

Study of yeasts-

I. Examine a small amount of yeast taken from an agar-slant in water under the high power of the microscope. Note the manner of reproduction by "budding."

2. Prepare a culture in liquid wort of Sacch. cerevisiae.

3. Pour the supernatant liquid of the 24 -hour-old culture off, and distribute the sediment on a gypsum block.

Note.-Gypsum blocks may be prepared in the following manner: Gypsum (plaster of paris) is mixed with half its volume of water and quickly placed in a cylinder of paper. When 


perfectly dry, the paper is cut away and the block is placed in a suitable vessel (a Stender dish or a deep, narrow Petri dish will answer very well). The block and vessel are then sterilized in the hot-air sterilizer for one hour.

4. Pour enough distilled water around the gypsum block to submerge about one-half of it.

5. Set aside in a cool, dark place for 3 or 4 days.

6. Examine a small portion of the film on the surface of the gypsum under the microscope in water.

Note.-Under very favorable conditions, and in the presence of plenty of oxygen, yeasts will undergo spore-formation. The great porosity of the gypsum block, which admits free communication with the water, and the fact that the surface of the block is exposed to the air, offer most favorable conditions for spore-formation, which takes place in 3 or 4 days.

EXERCISE II. THE STUDY OF THE GERMINATION OF SPORES

The germination of spores may be studied from spores (conidia) of mold fungi. Although somewhat different from the germination of bacterial spores, mold spores illustrate the general process very well.

I. Make a suspension of spores of Penicillium glaucum (common green mold) in broth.

2. Flame carefully a hollow-ground slide so as to exclude all other organisms, and paint vaselin around the hollow so as to form a complete ring.

3. Take a loopful of the spore suspension and place it on a clean flamed cover-glass.

4. Invert over the prepared hollow-ground slide, and gently press the edges on the vaselin so as to exclude all communication with the air. This prevents the hanging-drop from drying out, and also the invasion of foreign organisms. 
$5^{2}$ LABORATORY GUIDE IN BACTERIOLOGY

5. Observe under the microscope (No. 7 objective), and make sketches from day to day of the progress in the growth, until the complete organism is developed and spores are again formed. 




\section{CHAPTER IX}

SCHEME FOR ROUTINE STUDY OF THE VARIOUS GROUPS OF ORGANISMS

This scheme is to be followed strictly in the study of all organisms, except when special instructions are given, and the student should thoroughly familiarize himself with the different steps. The work of cultivation as described in steps I, 3 , and 5 may be divided between two students working together, but all other procedures are to be followed by each individual.

I. The inoculation of one slant-agar-tube from each individual stock-culture supplied. These inoculated agartubes are to be incubated at $37^{\circ}$ for 24 hours, unless otherwise instructed.

2. At the end of 24 hours make from each agar-tube:

a) A physical description of the culture (see Chap. $\mathrm{X})$.

b) A hanging-drop examination.

c) A Gram stained preparation.

d) An ordinary stained preparation.

Method of making preparations according to Gram-

a) Prepare a film of the organism to be examined, the same as in the ordinary stained preparation.

b) Cover with gentian-violet for I minute.

c) Wash in water, and remove the water by means of filter-paper, leaving the surface moist.

d) Cover with Gram's iodin solution for 2 minutes.

e) Pour Gram's iodin solution off and, without washing, place in a watch-glass, film side up, and cover with 96 per cent. alcohol. 


\section{LABORATORY GUIDE IN BACTERIOLOGY}

f) Allow to remain in alcohol, with occasional agitation, for at least 4 minutes, or until no more stain is taken up by the alcohol.

g) Dry without washing, and mount.

This stain is an important means of differentiating species of bacteria.

In order to be able to judge of the effect of Gram's iodin solution on the stain, the preparation before mounting may be washed in water and counterstained with Bismarck brown. This method shows all foreign matter brown in contrast to the bacteria, and is especially adapted for staining of bacteria in tissues, sputum, etc.

It is a positive Gram's stain if by application of this method either the organism loses none of the stain, or the stain is dark blue or dark slate-blue. It is a negative stain if either the coloration is completely gone, or only a light bluish tinge is left.

All stained preparations and all Gram stains must be preserved for inspection.

3. Transfer from agar-culture to the following media: Glucose-agar.

Gelatin.

Potato.

Broth.

Litmus milk.

Dunham's solution.

These transfers, excepting gelatin, are to be placed in the thermostat, unless otherwise ordered.

4. An accurate description is to be made of each individual culture (see Chap. X), and outline sketches especially of the gelatin and the milk cultures. These descriptions should be made complete after 24 hours, 


and any changes should be noted after 48 hours and after 6 days. (See culture charts.)

5. Plates are to be made in agar from a 24-48-hour-

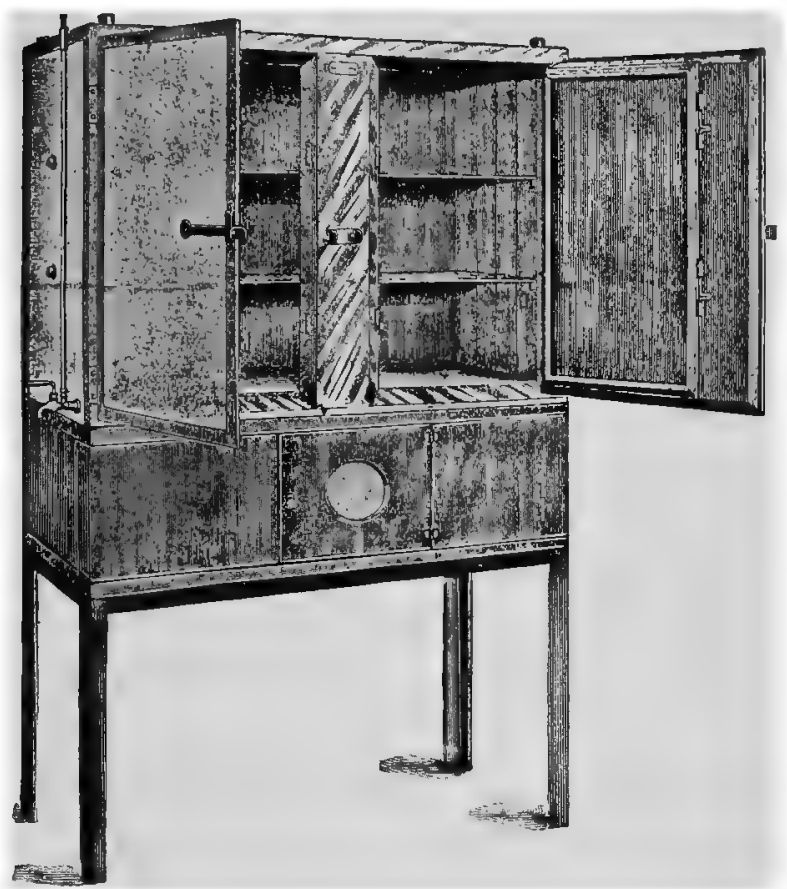

Fig. 26

Thermostat or Incubator

old broth-culture of each bacterium, unless otherwise directed. These plates are to be described once after 24-48 hours. (See Chap. X.)

Note.-The thermostat or incubator (Fig. 26) is a box made of copper and having double walls, between which water 


\section{$5^{6}$ LABORATORY GUIDE IN BACTERIOLOGY}

circulates. The outer surface is usually covered with asbestos or linoleum, so as to hold the heat. The thermostat is provided with two doors, the inner one of glass so as to enable the observer to look inside without opening it, the other one covered like the walls. A thermometer reaches through the upper part, and several air-holes are provided in it, which permit free circulation of air. The heat is controlled by Bunsen burners with selfregulating devices. The gas supply is shut off automatically, if it should accidentally become extinguished. For class use large incubators are constructed on the same principles, with a number of separate compartments. 




\section{CHAPTER $\mathrm{X}$}

\section{METHOD OF DESCRIBING CULTURES}

The following method of describing cultures should be carefully studied, and each suggestion should be considered in the description. It is of prime importance that all cultures in all media should be closely observed, and accurately described and sketched, as this is the only method which furnishes the proper means of studying and determining the different species of bacteria. By keeping this scheme in sight while making a description, it will be possible after a little practice to make perfect descriptions without its aid.

I. Morphological characters of bacterium. Size.

Facility and mode of staining.

Gram's stain.

Special staining qualities.

Motility.

Present or absent.

Sluggish or active movement.

Flagella present or absent.

Capsules present or absent.

Spores present or absent.

Involution forms.

Make an accurate sketch of part of a field under the microscope.

II. Plate cultures.

I. Naked-eye appearance.

a) Surface colonies. 
Approximate number.

Shape: punctiform, lanceolate, oval, circular, spindle-shaped, conglomerate, irregular, branched, filamentous, rosetteshaped.

Color in transmitted and reflected light.

Approximate diameter in millimeters.

Elevation (and shape of elevation) or depression.

Translucency.

Moist appearance.

Smoothness.

Luster.

Liquefaction (in gelatin plates only).

Consistency: soft, viscid, hard, chalky.

b) Deep colonies.

Color.

Shape.

2. Microscopic appearance under low power (No. 3 objective). Care must be taken to use very little light, the diaphragm should be almost closed.

a) Surface-colonies.

Shape.

Color.

Translucency.

Thickness (in center and edges).

Nucleation.

Striation.

Granulation (if present, whether coarse or fine).

Homogeneity. 


Tuberculation (tubercle-like appearances at edge).

Edge: entire or smooth, wavy, with pointed protuberances, serrate, dentate, lacerate (as if torn), fringed, hairlike shoots, curled.

b) Deep colonies.

Shape.

Color.

Translucency.

Granulation.

III. Agar-slant-culture.

Limitation: confinement to needle-track or spreading. If spreading, in what shape?

Vigor: luxuriant or scant.

Color: by transmitted and reflected light.

Elevation or depression. Elevation more pronounced at edges or in center.

Translucency.

Moistness.

Smoothness.

Luster.

Coloration of medium.

Pigment production.

Odor.

Gas-formation: in culture or in medium.

IV. Stab-culture in plain agar.

I. Surface-growth (describe like surface-colonies.)

2. Stab-growth.

Vigor.

Extent.

Color. 
Granulation.

Outgrowths.

Coloration of medium.

Cloudiness.

Gas-formation.

V. Stab-culture in glucose-agar.

Describe like plain agar, and in addition always note presence or absence of gas-formation, and presence of cloudiness.

VI. Stab-culture in gelatin.

Describe like plain agar, and in addition always note presence or absence of liquefaction. If liquefaction is present, it may be saucer-shaped, turnip-shaped, conical, funnel-shaped, horizontal (extending the whole diameter of tube), sack-shaped. Cloudiness and presence of sediment in liquefied area, and color and shape of sediment, should be described.

VII. Potato-culture.

Describe like agar-slant, adding to it the eventual discoloration of the medium.

VIII. Litmus milk culture.

Reaction (acid or alkaline, as indicated by color).

Coagulation: at ordinary temperature or upon heating.

Whey: if present, clear or turbid.

Liquefaction of coagulum (proteolysis, peptonization).

Gas-formation.

Decolorization of litmus (complete or incomplete). 


Color of cream-ring.

Odor.

In milk the presence or absence of coagulation and proteolysis should always be noted. Compare your culture always with a sterile control milk-tube.

IX. Broth-culture.

Cloudiness: degree and uniformity, scum: ringor island-shaped.

Precipitate, observed by shaking. Color, formation, diffusibility, viscidity, amount.

X. Solidified blood-serum.

Describe like agar-slant, and note presence or absence of liquefaction.

XI. Fermentation-tubes.

Gas-formation in closed arm, percentage and relation of carbon dioxid to hydrogen expressed by the formula $\frac{\mathrm{H}}{\mathrm{CO}_{2}}$.

Growth in both arms or in one arm only, observed by cloudiness.

- Reaction: acid or alkaline.

Directions for filling out the first page of culturecharts-(see Appendix III.)

I. Name the group and organism.

II. Read in the textbook or references given so as to indicate the source and habitat.

III. Name the most important references, and read them.

IV. Morphological characters.

I. Describe the morphology as observed from the stained preparation opposite the medium from which obtained. 
2. Size: approximate estimate in microns. Note whether large or small, thick or slender, round or square ends, etc.

3. Arrangement of bacteria: in groups, chains, bunches, pairs (diplococcus), sarcina form, threads, branching, etc. Also note different arrangements, if observed, in different media.

4. Staining powers: It is sufficient to mark + for positive, - for negative stains. Special stains must be described more fully.

5. Motility: If absent, mark - ; if present, + . In the latter case describe the character of the movement.

6. Spores: Absence noted by - ; presence, by + This information is to be gathered from the textbook, unless specially instructed. If so mention the method of spore-stain applied.

7. Note any peculiar appearance in the microscopical picture, especially involution forms and the presence or absence of capsules. If capsules are present, note the method of demonstration.

V. Physiological characters.

I. Relation to temperature: What is the optimum temperature? (See textbook or references.)

2. Relation to free oxygen: aërobe, anaërobe, facultative aërobe or anaërobe.

3. Relation to disinfectants, light, desiccation, heat (thermal death-point). (Refer to textbook or references.)

4. Pigment-production: If present, + ; if absent, -. In the former case, note the color, dif- 


fusibility, solubility, influence of acids and alkalis.

5. Gas-production in glucose media: To be filled out only in case of actual observation. In fermentation-tubes note the growth in either arm or both arms, recognized by turbidity. Note the total percentage of the gas formed in 24 and 48 hours. Reaction may be tested by the addition of litmus-solution. Gas formula expressed: $\frac{\mathrm{H}}{\mathrm{CO}_{2}}$.

6. Acid or alkali production in litmus milk.

7. The production of indol or nitrites, or both, is tested on the sixth day of observation in a culture in Dunham's peptone-solution or sugar-free broth.

NoTE.-Indol is a decompesition product of proteids and belongs to the aromatic series. Nitrites are the result of reduction from nitrates. The ability of organisms to produce these reactions are of great importance in their differentiation. A control test with a tube of sterile medium should always be made.

8. Enzym-production: Proteolytic enzym-production noted by the liquefaction of gelatin or casein. Coagulative, by precipitation of casein, if acid-formation is absent, or present only in quantities less than 0.3 per cent. Diastatic, by the digestion of starch (potato).

9. Characteristic odor.

Io. Pathogenesis: What pathogenic effect has the organism on man? What effect on animals, 


\section{LABORATORY GUIDE IN BACTERIOLOGY}

\section{and which animals? What diseases are}

caused by the organism in man or animal?

Note.-It may be well here to call attention to the terms "proteolysis," "enzym-production," and "coagulation."

"Proteolysis" is the breaking up of complex nitrogenous compounds (proteids), rendering them soluble. This process is also expressed by the terms "peptonization" and "liquefaction." The liquefaction of gelatin is one kind of proteolysis. Gelatin is composed of nitrogenous matter (albuminoid or gelatinoid), and it is for this reason mainly that gelatin stab-cultures are made. If the gelatin is liquefied, we conclude that the organism is capable of producing a "proteolytic" enzym. In milk the process is more complex, and this medium, on account of its composition (fat, milk-sugar, casein, lactalbumin), offers excellent opportunities for the organism to develop different characteristics. Milk is one of the most important media. The caseinogen, contained in milk in colloid solution, may be precipitated by an enzym or by an acid. This precipitate forms the coagulum. At least 0.4 per cent. of acid, which is largely lactic acid produced by splitting of milk-sugar (lactose), is required for precipitation, and this amount of acid will turn the blue litmus to a decided pink. A coagulum may also be produced by the presence of a "coagulative" or "rennet"-like enzym, which is the result of the metabolic activity of the organism. Such coagulation may take place in milk of an amphoteric or alkaline reaction, as well as in milk of a slightly acid reaction. The coagulum formed by any of the mentioned agents may gradually contract, and a strawyellow, opalescent liquid is squeezed out, called "whey." If, now, the organism also produces a proteolytic enzym, this will attack the coagulum and gradually dissolve it (proteolysis, peptonization). At first the coagulum shows a broken-up surface; lumps separate and settle to the bottom, and finally the coagulum may completely disappear. Theoretically, coagulation is always necessary before proteolysis sets in, but in the case of some organisms the proteolytic enzym is so powerful as to produce immediate dissolution of the casein.

Another phenomenon frequently observed in litmus milk is 


the discolorization of the litmus, whether this be pink or blue. This is due to the fact that the organism takes up the oxygen necessary to maintain the coloration. It may be frequently observed that at the surface, where atmospheric oxygen has access, the color remains or is restored. The color may also be restored by shaking the milk vigorously, thus bringing it into intimate touch with the oxygen of the air.

The production of a diastatic enzym (diastase, amylase) is demonstrated by gas-production on potatoes. This medium contains starch in large amounts. The starch is inverted into glucose by diastase (amylase), and this is then fermented with gas-production.

Directions for filling out second page of culture-charts-

I. Note the reaction of the medium by the sign + for acid and - for alkali reaction.

2. The incubation temperature may be (a) $37^{\circ}$ (thermostat), (b) room temperature, (c) ice-chest.

3. Plates are to be described only once after 24 or 48 hours, according to growth. Make notes in the first column for gelatin; in the second, for agar-plate, writing across the full width of the page. Make sketches in the special column reserved for this purpose.

4. The growth on the media $3,4,5,6,7$, and 8 (enter here: "glucose-agar" only unless otherwise instructed) to be made fully according to the outline in the spaces under 24 hours. In the spaces under 48 hours and 6 days note only the changes from the first day.

5. Make sketches frequently and accurately, especially from milk and gelatin media, and any other remarkable growth, in the column reserved for this purpose; also a sketch of each organism from a part of a field under the microscope.

It is well to understand that these directions for fill- 

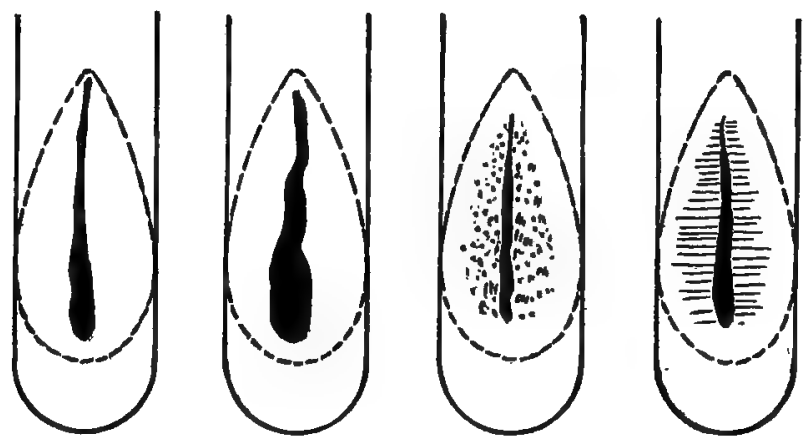

Fig. 27

Streak-Cultures
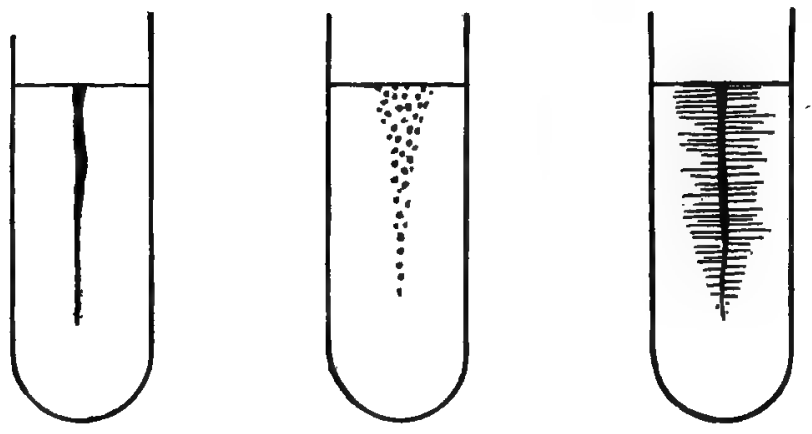

FIG. 28

Stab-Cultures 



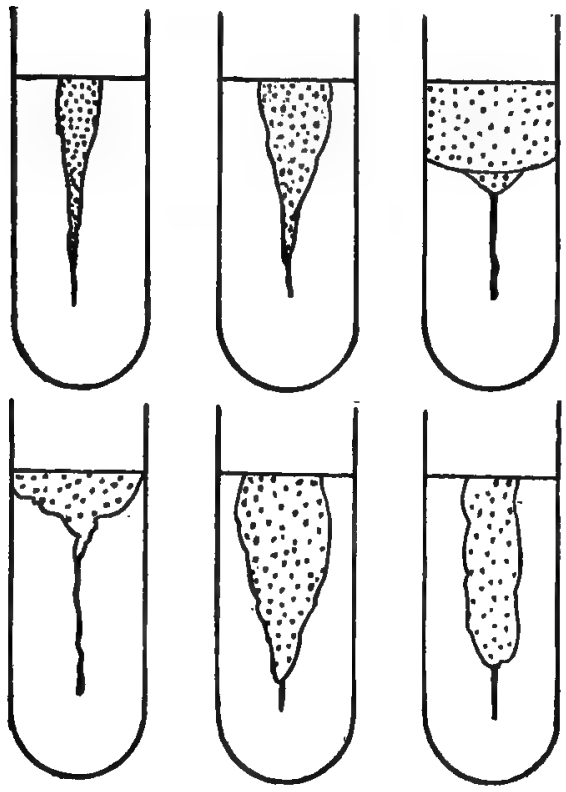

FIG. 29

Liquefaction of Gelatin

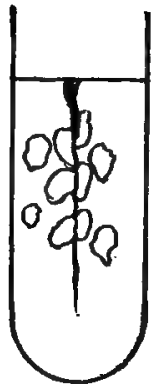

I

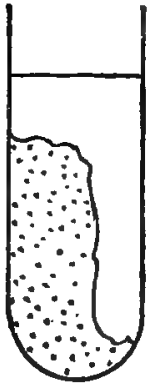

2

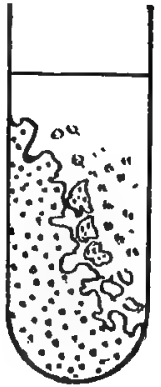

3

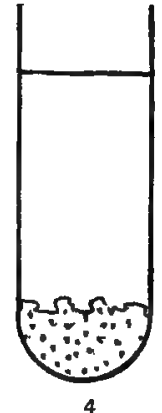

4

Fig. 30

1. Gas-bubbles in glucose-agar

z. Coagulation of milk
3. Coagulation and peptonization of milk

4. Complete peptonization of milk 
ing out culture-charts are applicable in the described manner to studies of cultures furnished by the laboratory. For determining any species of unknown bacteria, original researches must be made to cover all points, necessarily without the possibility of gathering this information from textbooks or references. On the accuracy of observation and description depends the success of bacteriological work and species-determination. It is frequently necessary to employ special media, or inoculation of animals, or such reactions as the agglutination test, to determine properly what species one is dealing with.

The student will do well to familiarize himself carefully with all directions and explanations given in this and the previous chapters. Find a characteristic for each item mentioned; otherwise the descriptions will be incomplete; and follow the instructions for routine work with all possible accuracy. 




\section{CHAPTER XI \\ STUDY OF CERTAIN CHROMOGENIC BACTERIA}

EXERCISE I. CULTURAL STUDIES

I. Read carefully the following references to chromogenic organisms, and the production and chemistry of pigments:

Sternberg, Manual of Bacteriology.

Lehmann and Neumann.

Members of this group are widely disseminated in the air, water, etc. A few representatives will be studied.

2. Inoculate agar-slants from stock-cultures of Bacillus prodigiosus, B. pyocyaneus, B. violaceus, and Sarcina lutea. Inoculate three slants each of $B$. prodigiosus and $B$. pyocyaneus, and one each of Sar. lutea and B. violaceus.

3. Label each tube with the name of the culture inoculated, the date of the stock-culture and the date of inoculation. Place the label on the side of the tube where the slanted surface of the medium is. It is then possible to study the growth and see the label at the same time. Glass pencils for marking are very convenient.

4. Place one culture of each organism in the thermostat, one culture of $B$. prodigiosus and $B$. pyocyaneus in the locker, and leave the others exposed to sunlight.

5. After 24 hours compare the growths of B. prodigio. sus and $B$. pyocyaneus, under the various conditions, in respect to-

a) Relative amount of growth.

b) Relative amount of pigment produced.

What conclusions can be drawn from this experiment? 
6. Note the characteristics of the pigments: Are they diffused through the medium, or are they confined to the growth?

7. Make descriptions of agar-cultures; also hangingdrop, stained, and Gram preparations.

8. Transfer from 24-hour-old agar-cultures of all organisms to all media. (See routine study, Chap. IX.) Potatoes may be inoculated with the looped needle, as the surface is too rough to allow of a smooth inoculation with the straight needle.

9. After all cultures have been in the thermostat for 24 hours, make all descriptions as outlined in Chap. X. (Record after 48 hours and 6 days only observations of change.)

CAUTION.-The gelatin-cultures are sometimes placed by students in the thermostat along with others through oversight. This, of course, completely defeats the purpose of obtaining a stab-growth, as the gelatin will melt. In order to avoid this mistake, it is recommended to label one tin cup or tumbler "Gelatin" in large letters. This will serve as a constant reminder that gelatin has to be kept at room temperature.

ro. Make plate-cultures of the four organisms.

Method of making plates-

a) Melt two agar-tubes for each organism in the waterbath and cool to $43^{\circ}$.

b) Transfer 3-5 loopfuls (according to the intensity of the growth, to be judged by the degree of cloudiness) of the broth-culture to a sterile tuhe of Dunham's solutions. (Dilution I).

c) Shake well, avoiding air-bubbles as much as possible.

d) Transfer 4 or 5 loopfuls from this suspension to a melted agar-tube, and label this 2. (Dilution 2). 


$e$ ) Shake this carefully by rolling the tube between the palms of the hand, or stir with the platinum needle, so as to avoid air-bubbles.

D) Transfer 4 or 5 loopfuls of this agar-tube (2) to the second agar-tube (3), and mix as above.

g) If thought necessary, more tubes may be treated in the same way, resulting in still higher dilutions. In the meantime the inoculated tubes should be replaced in the water-bath, so as to keep them liquid.

h) Pour the contents of the tubes, one after the other, into sterile Petri dishes.

i) Tip the Petri dishes very carefully, so as to distribute the medium evenly over the bottom.

k) Label them and, 3 d, dilution, etc., with the name of the organism and the date.

$l$ ) Set aside on a level place to solidify.

$m$ ) When solidified, place them bottom $u p$ in the thermostat, in order to avoid moistening of the surface by the condensation water dropping down from the cover.

NoTE. -If the surface were moistened, the colonies would run together and the characteristic appearance be destroyed. Gelatin plates, on the contrary, are placed cover up. Condensation water does not form on these plates as gelatin may be liquefied by the organisms. The liquefied part would then fall from the medium on the cover and ruin the plate.

The plates prepared in the above manner should be studied after 24 hours, or, if not sufficiently developed, after 48 hours, according to directions in Chap. X.

EXERCISE IT. STUDY OF PIGMENTS

On the sixth day take agar-slant or potato-cultures of the four chromogenic bacteria, and proceed as follows: 


\section{LABORATORY GUIDE IN BACTERIOLOGY}

Pour 96 per cent. alcohol on the cultures of $B$. prodigiosus, B. violaceus, and Sar. lutea. The pigment should dissolve. Filter the liquids into clean test-tubes, and, by adding a few drops of 5 per cent. hydrochloric acid, note the change in color. Then add an excess of a 2 per cent. solution of sodium hydrate, and note whether or not the color returns and is changed again. Then pour chloroform on a culture of $B$. pyocyaneus. This will dissolve the bluish-green pigment (pyocyanin). Filter, and evaporate on the water-bath. When almost dry, place a small amount on a slide, and observe the small crystals of pyocyanin under the microscope. Note also the aromatic odor given off by the pigment as the solvent evaporates. 




\section{CHAPTER XII \\ THE PYOGENIC GROUP}

EXERCISE I. THE PYOGENIC GROUP (SUBGROUP A) ${ }^{\mathrm{x}}$

\section{MEMBERS-}

Staphylococci, streptococci, Microc. tetragenus.

Inoculate agar-slants from cultures (furnished) of Staphylococcus pyogenes aureus, Staph. pyogenes albus, and Streplococcus pyogenes. These organisms are pathogenic, and care must be taken to observe the rules of technique with the utmost accuracy. Any carelessness may be followed by the gravest consequences. In case of accident, such as the spilling of a culture or infecting the hands, thorough disinfection-e. g., with a solution of mercuric chlorid (I: IO00)-is absolutely necessary.

After 24 hours' incubation of the three agar-slants, proceed with the other media as outlined in the routine study (Chap. IX).

Special study.-A rabbit will be inoculated intravenously with a broth-culture of Staph. pyogenes aureus. The ear of the rabbit is carefully shaved, washed with mercuric chlorid solution, followed by alcohol. Then 0.5 c.c. of a 24-hour-old culture in broth is drawn up into a hypodermic syringe, which has been previously sterilized by immersion in boiling, water for to minutes. The mode of holding a rabbit is as follows: The left arm of an assistant rests against the hindquarters of the rabbit on the table, while the two hands

$r$ This subdividing of the pyogenic group is an arbitrary measure designed simply to facilitate study, the commoner pyogens being studied first. 
gently hold the fore-legs. If the animal struggles, too much force should not be applied, as this might injure or kill it. The struggles may be overcome by wrapping the animal in a towel or any other similar piece of cloth. The needle is then inserted into the lumen of the lower vein (ramus lateralis posterior of the vena auricularis posterior), which has been pinched between the fingers, or by means of a forceps, so as to arrest the circulation. No air should be injected with the culture, as this will kill the animal. The hypodermic needle is then withdrawn and sterilized in boiling water for 15 minutes.

After the death of the rabbit, study the lesions produced by the organism, and make cultures on slant-agar, and smears from the heart's blood, spleen, and any foci of suppuration.

AUTOPSY (SEE SKETCH, FIG. 3I)

I. Have the instruments sterilized in boiling water.

2. Tie the animal by the extremities on a square board, with the abdomen upward.

3. Note the presence of any external lesions, such as swellings, ulcerations, etc.

4. Wash with a solution of mercuric chlorid ( $\mathrm{I}: \mathrm{I}, 00)$ followed by alcohol.

5. Lift the skin over the pubes with the forceps, and with the scissors make an incision along the median line well above the sternal notch; then diagonal incisions extending to the fore- and hind-legs.

6. Cut the skin away carefully with a moderately sharp knife, avoiding opening the abdominal cavity, and pin to the board.

7. Open the abdomen by a median incision from the pubes to the sternum. 




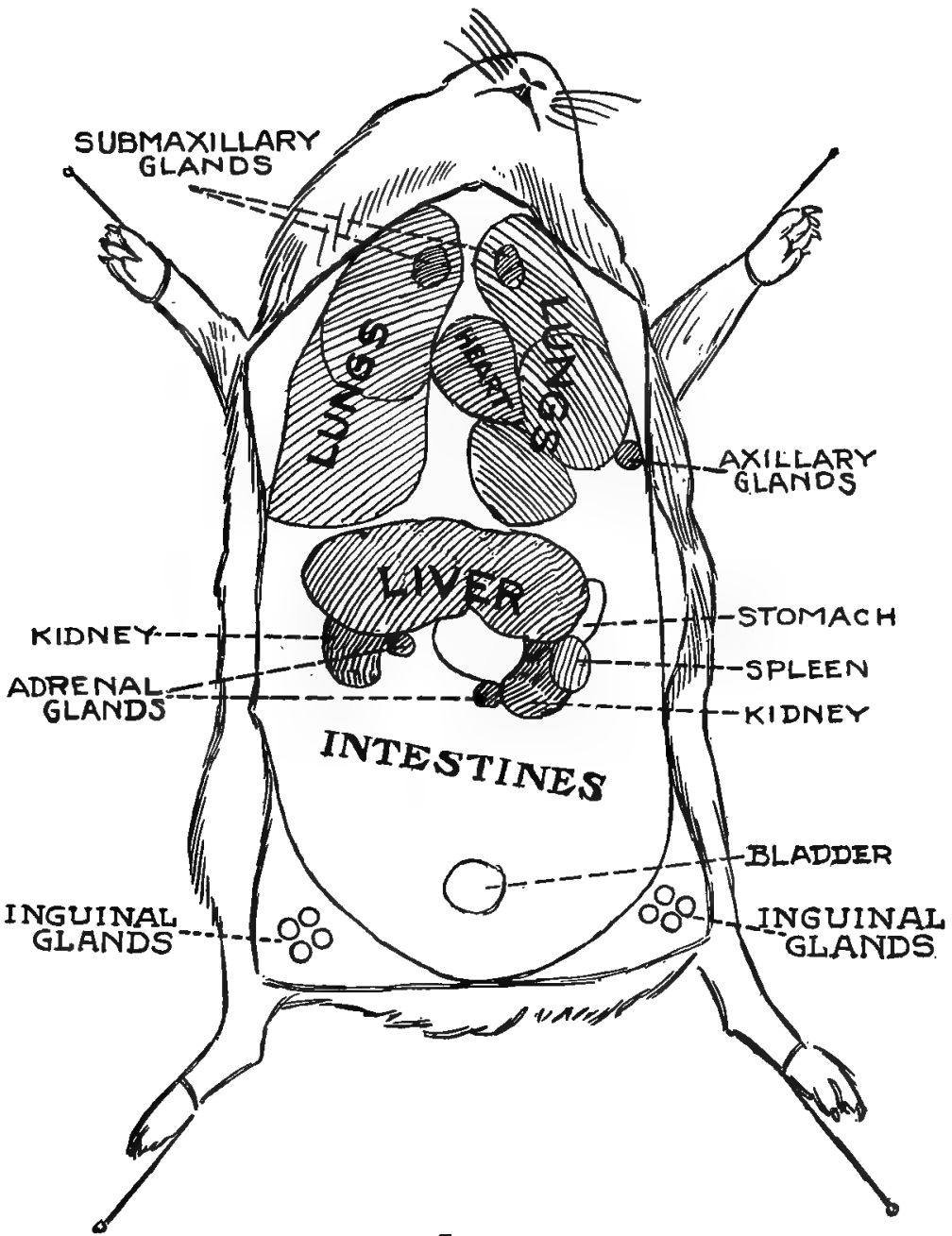

FIG. 3I

Method of Aulopsy of a Guinea-Pig (Diagrammatic) 
8. Remove the anterior thoracic wall by cutting away the ribs from below upward on each side to the thoracic apex.

The viscera are now thoroughly exposed. Cultures and smears should be made from different parts, especially from the heart's blood, peritoneal cavity, spleen, liver, and localized foci of suppuration.

EXERCISE II. THE PYOGENIC GROUP (SUBGROUP B) Members-

Micrococcus lanceolatus.

Micrococcus gonorrhoeae.

Micrococcus intracellularis meningitidis.

Micrococcus zymogenes.

Prepared cover-slips of Micr. gonorrhoeae are furnished. Stain these with methylene-blue and Gram's method, study, and describe the microscopical appearance. This organism is extremely difficult to cultivate. It is a strict parasite, and requires special media, and a great amount of time and care, for artificial cultivation. For these reasons, only the morphology, as it appears in gonorrheal pus, and which is very characteristic, is studied.

Inoculate agar-slants from stock-cultures of $\mathrm{Micr}$. lanceolatus and $M i \mathrm{cr}$. zymogenes.

REFERENCES (Micr. zymogenes)-

MacCallum and Hastings, Journal of Experimental Medicine, Vol. IV (1899), p. 52 I.

Harris and Longcope, Centralblatt für Bakteriologie und Parasitenkunde, Part I, Vol. XXX, No. 9 (printed in English).

I. Routine study.-Note particularly the microscopic appearance of both organisms and the action of $M \mathrm{icr}$. zymogenes on milk and gelatin.

2. Special study A.-The staining of capsules from 


a milk-culture of Micr. lanceolatus. Two methods may be applied for this stain:

First method (Friedländer's method):

a) Prepare a stain by the following formula:

Glacial acetic acid............... I part

Saturated alcoholic solution of gentian-

violet.................. 5 parts

Distilled water................... Io parts

b) Prepare a very thin film in the usual manner from a 24-hour-old milk-culture, taking care to spread very thinly.

c) Cover with stain for ro-I5 seconds.

d) Wash in a solution of sodium chlorid ( 0.85 per cent.).

e) Examine in a sodium chlorid solution.

f) If satisfactory, float the cover-slip off with salt solution, dry, and mount in balsam. Under no conditions should plain water come in contact with this preparation at any stage; otherwise no capsules will appear.

Second method (Welch's method):

a) Prepare a very thin film in the usual manner from a 24-hour-old milk-culture.

b) Cover with glacial acetic acid for 5 seconds.

c) Wash acid off with carbol-fuchsin.

d) Wash stain off with a 0.85 per cent. sodium chlorid solution.

e) Examine in salt solution.

f) If satisfactory, mount in balșam.

Plain water should not come in contact with this preparation at any stage. The capsule should be observed as a lightly stained zone with well-defined outline around the deeply stained organism. 
3. Special study B.-Inoculation of mouse with $M i c r$. lanceolatus.

a) Fasten the mouse in the holder (Fig. 32).

b) Shave a place on the back immediately above the tail.

c) Wash with a solution of mercuric chlorid ( $\mathrm{I}: \mathrm{I} \infty \infty)$, followed by alcohol.

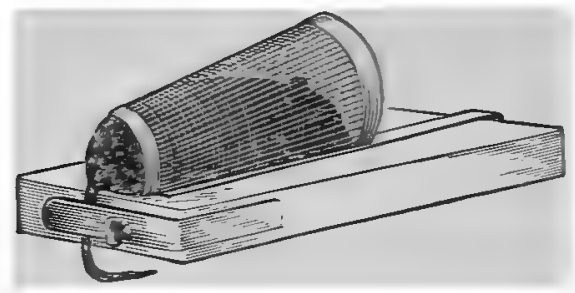

Fin 3

Moure-Holiter

d) Inject 0.2 c.c. of a milk-culture of Jicr. lanceolatus.

e) When dead, perform an autopsy, and study the lesions in the usual manner.

f) Make cultures in milk and on slant-agar from the heart's blood or the spleen.

g) Make a capsule stain from the heart's blood, spleen, or other organs. 




\section{CHAPTER XIII}

\section{THE INTESTINAL GROUP}

This chapter is devoted to the study of the "intestinal group" of organisms. This collective group may conveniently be subdivided into four subgroups:

Subgroup I: the colon group.-This group includes different varieties of Bacillus coli, B. lactis aërogenes, and some species of $B$. acidi lactici.

Subgroup 2: the hog-cholera, $B$. enteritidis, or intermediate group.-This group includes $B$. cholerae suis, $B$. paratyphosus (several varieties), $B$. enteritidis, and $B$. icteroides. The term "intermediate" is assigned to this group, because it resembles the colon group on the one hand, and the typhoid group on the other.

Subgroup 3: the typhoid-dysentery group.-This group includes $B$. typhosus, varieties of $B$. dysenteriae, and $B$. faecalis alcaligenes.

Subgroup 4: the proteus group.-This group includes all varieties of proteus (Bact. termo) and B. cloacae.

EXERCISE I. STUDY OF SUBGROUP I: THE COLON GROUP

Inoculate agar-slants from stock-cultures of $B$. coli (two varieties) and $B$. lactis aërogenes. Also inoculate one tube of broth with $B$. coli.

These two types of $B$. coli are differentiated by their ability to ferment carbohydrates:

A. Those which ferment dextrose, lactose, and saccharose.

B. Those which ferment dextrose and lactose, but not saccharose. 


\section{REFERENCES-}

Theobald Smith, The Wilder Quarter Century Book, I893, p. 187 .

Jordan, Journal of Hygiene, Vol. I (Igor), p. 295.

Durham, Journal of Experimental Medicine, Vol. V, p. 353.

I. Routine study.-Observe carefully the growth on potato of $B$. lactis aërogenes. What is the cause of gasformation in this case?

2. Special study $A$.--In order accurately to test the action of micro-organisms on various carbohydrates, it is necessary to eliminate the small amount of sugar in ordinary broth introduced into it by meat-extract, which generally contains muscle-sugar (glycogen). This is accomplished by adding to freshly prepared broth a culture of $B$. coli, which completely decomposes many carbohydrates, including muscle-sugar. By this method a sugar-free broth is prepared, which may be used as a solvent for any sugar desired.

Preparation of sugar-free broth for the fermentationtube:

\section{Dissolve-}

In water 400 c.c.

Extract of beef................. I $\mathrm{g}$.

Peptone.................... 4 g.

by heat. Broth made from chopped beef (500 g. to I liter) may also be used for this purpose.

2. After cooling, inoculate with a broth-culture of $B$. coli prepared 24 hours previously.

3. Set aside in the locker, or, better, in the thermostat, for $18-24$ hours.

4. Boil 5 minutes (to kill $B$. coli), and filter repeatedly through the same paper until perfectly clear.

5. Divide into three equal parts and dissolve $2 \mathrm{~g}$. 


(I $\frac{1}{2}$ per cent.) dextrose, lactose, and saccharose, respectively, in each part, and filter again, if necessary.

6. Fill fermentation-tubes, taking care to label each one properly, and sterilize in Arnold on 3 consecutive days for 20 minutes.

All gas must be carefully tilted out of the closed arm of the tube while the fluid is warm. When sterilization is completed, inoculate one set of the fermentation-tubes with $B$. coli $\mathrm{A}$, another set with $B$. coli $\mathrm{B}$, and a third set with $B$. lactis aërogenes. Inoculate with the straight or looped needle.

After 24 hours, note the percentage of gas formed. This is done by means of Frost's fermentation-chart, a model of which is represented by Fig. 33. This chart is placed between the open and closed arms of the tube, resting on the neck and moved along until the extreme upper end of the closed arm is level with the top of the chart and parallel with the vertical lines. The percentage may then be read off by the figures marked at each end of the chart.

After taking note of the percentages, replace the fermentation-tubes in the thermostat, and repeat the measurement after another 24 hours' incubation. Many organisms are able to obtain the oxygen necessary for life by attacking compounds-as, for instance, carbohydrates-and decomposing them. In such cases a turbidity is developed in the medium. The closed arm offers strict anaërobic conditions, all the atmospheric oxygen having been driven out by the heat of sterilization. The bulb allows the organism to come in contact with atmospheric oxygen, and therefore offers aërobic conditions.

Gas-production is by no means a constant phenom- 


\section{LABORATORY GUIDE IN BACTERIOLOGY}

enon accompanying a fermentation. Carbohydrates are fermented by many organisms without gas-formation. The usual product in this case is an acid, mostly lactic acid. Such fermentations produce turbidity only, and no gas. It is therefore necessary, in describing them, to characterize the process as fermentation without gas-formation.

Method of analyzing the gas produced in the closed arm. -The gas consists approximately of carbon dioxid and hydrogen, which may be proved by the following method: Fill the bulb with a 2 per cent. solution of sodium hydrate, and close the mouth with the thumb, taking care not to leave any air between the thumb and the liquid. Now slowly tilt the gas back and forth from the closed arm to the bulb five or six times, and finally allow it to collect again in the closed arm. The sodium hydrate combines with the carbon dioxid, and consequently on releasing the thumb the volume of gas will become smaller in proportion to the amount of carbon dioxid absorbed. Now measure the percentage again with the chart, and ascertain the proportion of gas left in the closed arm to the original amount.

\section{Example-}

Total percentage of gas before addition of $\mathrm{NaOH} \ldots 45$

Percentage left after absorption by $\mathrm{NaOH} \ldots \ldots \ldots 3^{\circ}$

Difference...................... I5

30 per cent. then represents the amount of hydrogen left, and 15 per cent. the amount of carbon dioxid absorbed. The proportion is expressed by the formula

$$
\frac{\mathrm{H}}{\mathrm{CO}_{2}}=\frac{30}{\mathrm{I} 5}=\frac{2}{\mathrm{I}} \text {. }
$$




The fact that this gas remaining in the closed arm is hydrogen may now be proved by tilting it into the open arm, which has previously been filled with water and closed by the thumb. By holding a burning match over it and quickly releasing the thumb, a slight explosion takes place from the sudden combination of the hydrogen with the oxygen of the atmosphere.

Gas-formation by bacteria does not necessarily depend on the presence of carbohydrates. Nitrogen is often produced from nitrites, and hydrogen sulphid and ammonia from proteids, especially during the process of putrefaction.

3. Special study B.-Test for indol and nitrites. What is indol? What biological activity of the organism does the presence of indol or nitrites, or both, indicate?

a) Test for nitrites: Add to a culture in Dunham's solution, or, better, in sugar-free broth, successively I drop of each of the following solutions:

(I) Sulphanilic acid.................

Acetic acid $(25 \%) \ldots \ldots \ldots \ldots \ldots \ldots+150$ c.c.

(2) Naphthylamine chlorid............. $0.1 \mathrm{~g}$.

Distilled water................... 20 c.c.

Acetic acid $(25 \%) \ldots \ldots \ldots \ldots \ldots \ldots \ldots$. 150 c.c.

A yellowish-red or rose-color shows the presence of nitrites.

b) Test for nitrites and indol combined.

(I) Add to a culture in Dunham's solution, or sugarfree broth, I or 2 drops pure sulphuric acid.

(2) Heat gently. Rose-color shows the presence of nitrites and indol. If no reaction takes place, add-

(3) A few drops of a solution of $0.1 \mathrm{~g}$. potassium or sodium nitrite in 1000 c.c. water. Rose-color then indicates the presence of indol only. 


\section{LABORATORY GUIDE IN BACTERIOLOGY}

Perform these tests with all the organisms of the intestinal group, and make control tests in sterile Dunham's solution.

4. Special study C.-Make capsule stain of $B$. lactis aërogenes from 24-hour-old milk-cultures. (For method see P. 77.)

The study of $B$. coli is of special importance in connection with bacteriological analyses of water (see Chap. XXIV). The presence of this organism in large numbers indicates sewage contamination, and consequently the danger of an admixture of pathogenic bacteria such as B. typhosus and B. dysenteriae.

\section{EXERCISE II. STUDY OF SUBGROUP II}

THE HOG-CHOLERA, B. ENTERITIDIS; OR INTERMEDIATE GROUP

Use great care in handling members of this group.

Inoculate agar-slants from stock-cultures of $B$. cholerae suis (bacillus of hog-cholera), $B$. enteritidis (Gärtner's bacillus), and B. paratyphosus (two strains).

REFERENCES-

B. cholerae suis:

Moore, The Pathology of Infectious Diseases of Animals.

McFarland, Textbook of Bacteriology.

B. paratyphosus:

Buxton, Journal of Medical Research, 1902, Vol. VIII, p. 201. Wells and Scott, Journal of Infectious Diseases, 1904, No. I. Cushing, Johns Hopkins Hospital Bulletin, 1900, p. 156.

Durham, Journal of Experimental Medicine, Vol. V, p. 353.

I. Routine study.-Observe particularly the bluishgreen coloration of the cream-ring in litmus milk, and make test for indol.

2. Special study A.-Inoculate plain sterile milk with $B$. cholerae suis. After 8-ro days a clearing of the milk 


will be observed, due to a solvent action of the alkali produced by the organism upon the proteid content.

3. Special study B.-Inoculate fermentation-tubes the same as for $B$. coli. Measure and test gas. Compare the results with those obtained in the study of the colon group. What difference do you notice?

4. Special study C.-Inoculation of a rabbit subcutaneously with $B$. cholerae suis. Subcutaneous inoculations of rabbits are usually made in the following manner: An assistant, in a sitting position, places the rabbit back-down in his lap. The head projects beyond the knees of the assistant. The ears and hind-legs are grasped, and the animal is thus held in position. The hair is then cut off on a portion of the abdomen, and the place is treated with mercuric chlorid and alcohol in the usual manner. With sterilized fingers the skin is then pulled up, the syringe inserted, and the material injected.

After the rabbit has died, study the lesions produced by the organism, and make smears from the site of the inoculation, the heart's blood, and other organs. Note the polar staining, i. e., stained portions at the two ends of the cell and an unstained area between. Make cultures on agar from the heart's blood and other internal organs.

EXERCISE III. STUDY OF SUBGROUP III THE TYPHOID-DYSENTERY GROUP

Use great care in handling members of this group.

Inoculate agar-slants from stock-cultures of $B$. typhosus, B. dysenteriae (Shiga), and B. faecalis alcaligenes.

r. Routine study.-Study carefully the reaction on milk, and test for indol. Preserve glucose-agar-cultures for two weeks. 
2. Special study A.-Inoculate fermentation-tubes in the same manner as in the two preceding groups. $\mathrm{Ob}$ serve the absence of gas-formation but note growth in both arms. Compare the results with those of the colon and intermediate groups.

3. Special study B.-The staining of flagella.-Read carefully in the textbook the methods of staining flagella. To demonstrate the presence of flagella on $B$. typhosus, the following method will give good results (Loeffler's method):

a) Prepare the mordant. (What is a mordant? See the textbook.)

Tannic acid (25\% aqueous solution).......... ro parts Ferrous sulphate (saturated aqueous solution)... 5 parts Fuchsin (saturated alcoholic solution)......... I part

b) Prepare a number (six) of cover-slips by carefully flaming them, and place them side by side on a piece of filter-paper. (This paper must be burned after using).

c) Place 4 or 5 loopfuls of water on a clean slide.

d) Make a light suspension in this water of $B$. typhosus from a 24-hour-old agar-culture, taking particular care to stir the suspension as little as possible.

e) Place a loopful of water on each of the cover-slips.

f) Carry over a loopful of the suspension on the slide to one of the cover-slips, from this to second, from the second to the third, etc.

g) Spread carefully and allow to dry in the air.

h) Cover with the mordant.

i) Heat over a small flame for $I \frac{1}{2}$ minutes while steam rises, or better heat for 5 minutes on a water-bath. Replace the evaporated stain, to prevent its drying on the cover-slip. 


j) Wash thoroughly in water.

$k$ ) Drain the water off with blotting-paper.

l) Cover with anilin-gentian-violet or carbol-fuchsin.

$m$ ) Heat as before over a small flame for $\mathrm{I} \frac{1}{2}$ minutes.

$n$ ) Wash thoroughly in water.

o) Examine in water.

p) If satisfactory, mount in balsam.

4. Special study C.-Agglutination.-Dried-blood method of Johnston: A drop of blood of a typhoidfever patient is obtained by pricking the lobe of the ear, previously carefully cleaned and washed with alcohol. The blood is taken up by a piece of sterile non-absorbent paper or on a sterile aluminum slide. This is sent to a laboratory, where the blood is dissolved in physiological salt solution in such a manner as to obtain an approximate dilution of $\mathrm{I}: 25$. This solution is then tested with a suspension of typhoid bacilli, a young culture of which is constantly kept on hand for this purpose.

For laboratory tests the serum of an animal (either a rabbit or a guinea-pig) which has been injected with cultures of B. typhosus, previously heated for I hour at $60^{\circ}$. This process kills the organisms, but the toxins remain active. The animal is then bled in the following manner: One of the ears is shaved, and the skin is washed with alcohol. A small vein near the border is opened, and the blood is collected in a sterile glass vessel. If the animal does not bleed freely enough, it must be warmed, preferably by means of a hot-water bag. The blood is placed in the ice-chest, and the serum is collected after separation.

The method of procedure with a serum obtained in the above-described manner is as follows: 
a) Small quantities of the serum are diluted with sterile salt solution ( 0.85 per cent.) so as to represent dilutions of $I: 5, I: 25$, and $I: 50$.

b) A suspension of a 24-hour-old agar-culture of $B$. typhosus in salt solution is prepared. This suspension should be faintly turbid and uniformly so.

c) Three hanging-drop preparations are made by mixing a loopful of this suspension, with a loopful of the three serum dilutions, respectively. The final dilutions then are: $I: 10,1: 50$, and $I: 100$.

d) Examine with the high power (dry lens), and observe the clumping of the bacilli, preceded by the loss of motility.

e) Tabulate the results as to time and completeness of reaction.

f) Make a control hanging-drop without serum to test the motility and the absence of clumps.

Blood may also be obtained by puncturing the lobe of the ear and collecting it in a capillary glass tube with a small bulb. By holding the bulb down, fill threefourths full with blood, and seal the ends in the flame. In 45 minutes the serum will have separated, and is to be tested in the above manner.

The above-described method of agglutination test is known as the microscopic test. Another method, in which larger amounts of serum and suspension are required, is known as the macroscopic method. Small test-tubes are used, and definite amounts of bacterial suspensions are introduced by means of finely graduated sterile pipettes. The serum is then added in varying amounts so as to effect the desired dilutions (see table, $p$. II 7). The tubes are then incubated at $37^{\circ}$, usually for 


2 hours, or longer if desired. If complete agglutination takes place, the bacteria will have collected in clumps at the bottom, forming a sediment. The supernatant fluid is perfectly clear. By varying amounts of sediment and varying degrees of turbidity of the supernatant fluid, the degree of agglutination may be estimated. A controltube of a bacterial suspension without addition of serum serves as a guide. Controls with normal serum should also be made.

5. Special study D.--

a) Make cover-slip preparations of $B$. dysenteriae from glucose-agar-cultures IO-I2 days old. Involution forms are then plentiful and can be studied.

b) Also make cover-slip preparations of B. typhosus from glucose-agar-cultures Io-I 2 days old. Does it show the same picture?

EXERCISE IV. STUDY OF SUBGROUP IV

THE PROTEUS GROUP

\section{REFERENCES-}

Lehmann and Neumann.

Jordan, State Board of Health of Massachusetts, 1890 .

Jordan, Journal of Hygiene, Vol. II.

Inoculate agar-slants from stock-cultures of Proteus vulgaris, Prot. zenkeri, and Bacillus cloacae.

I. Routine study.-Observe particularly the action on milk and gelatin.

2. Special study A.-Make plates in gelatin and agar, and observe the colonies after 24,48 , and 72 hours. Note the appearance of the colonies of Prot. vulgaris and of Prot. zenkeri on both media.

3. Special study B.-Inoculate fermentation-tubes in the same manner as for the other intestinal groups. 
Determine the percentage of gas formed and the gas formula. Compare the results with those of the other intestinal groups. How does the gas formula differ from those previously examined?

Note.-In order to obtain a clear picture of the chief differential characteristics of the four intestinal groups, it is recommended to tabulate the results in parallel columns, as outlined below. Express positive results by the sign + ; negative, by - . Complete agglutination is expressed by ++ ; slight, by + . 




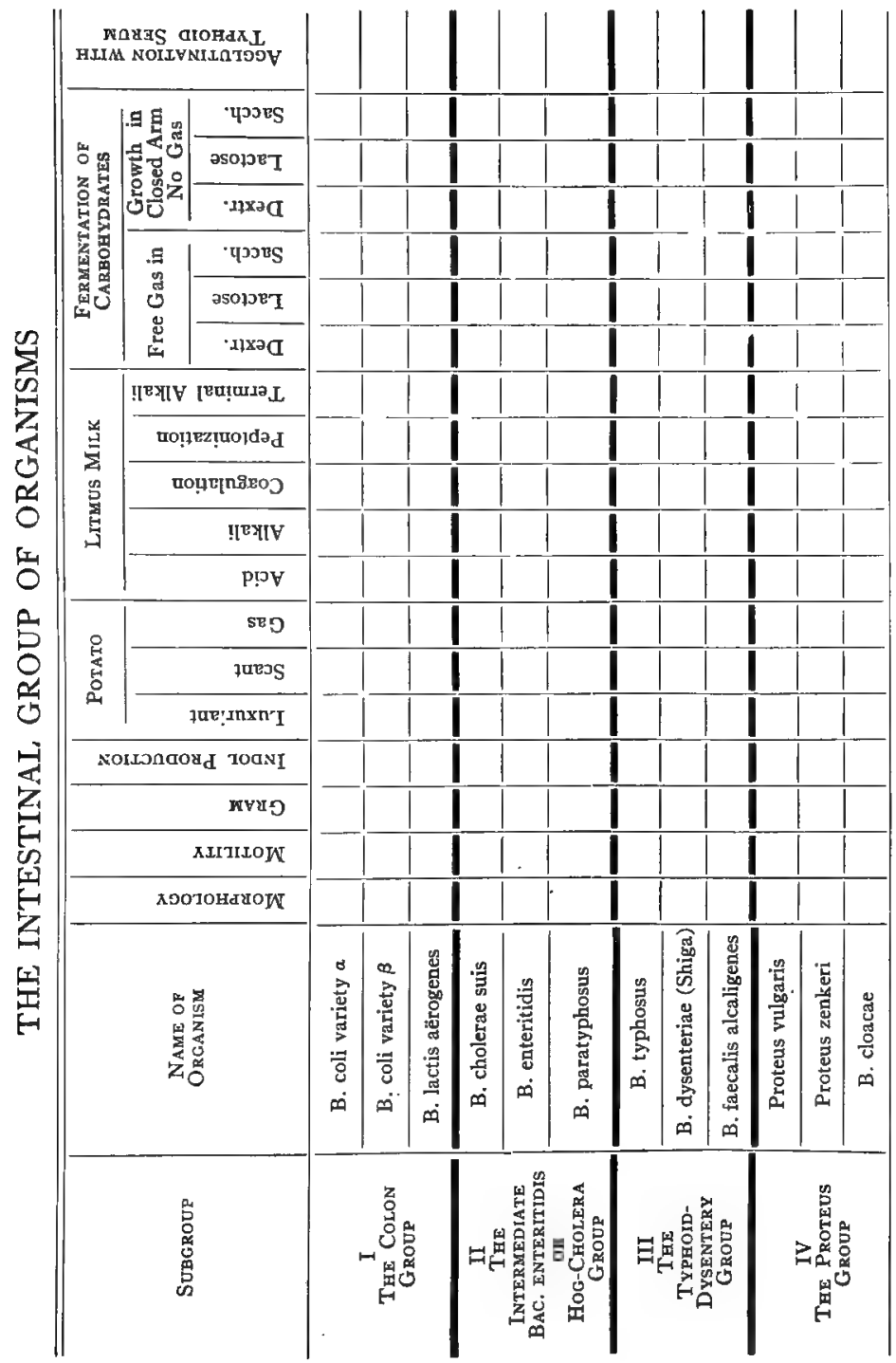




\section{CHAPTER XIV \\ THE CAPSULATED GROUP}

Members-

Varieties of Bacillus mucosus capsulatus.

$B$. lactis aërogenes.

REFERENCE-

Perkins, Journal of Infectious Diseases, I904, No. z.

Inoculate agar-slants from stock-cultures of $B$. mucosus capsulatus (Friedländer's pneumo-bacillus) and $B$. lactis aërogenes."

I. Routine study.-Observe particularly the viscous condition of cultures on solid media, the consistency of liquid media, and the gas-formation on potato.

2. Special study A.-Staining of capsules from 24hour-old milk-cultures (see p. 77).

3. Special study B.-Intraperitoneal inoculation of rabbit with $B$. mucosus capsulatus.

Method of intraperitoneal inoculation.-The rabbit is held in the same manner as described in Chap. XII. The hair is clipped close over the left lower abdominal quadrant. Then (after washing with mercuric chlorid I : I000 and alcohol) pass the needle at first beneath the skin, then, holding it at about a right angle to the abdominal surface, carefully press it through the abdominal wall, which is usually made tense by the resistance of the animal. Successful passage of the abdominal wall can be felt by the sudden loss of resistance to the needle's pressure. Then make the injection, and rapidly with-

I $B$. lactis aërogenes, if studied in the colon group, need not be studied again. 


draw the syringe. If the contents of the needle have been properly emptied into the peritoneal cavity, no swelling takes place, as is noticed in subcutaneous inoculations.

When the animal has died, perform an autopsy and study the lesions. Make cultures from the heart or internal organs in the usual manner, and make capsule stains from the heart's blood. 


\section{CHAPTER XV \\ THE DIPHTHERIA GROUP}

Members-

Bacillus diphtheriae.

B. pseudodiphthericus.

B. xerosis.

Use great caution in handling members of this group. Inoculate agar-slants from stock-cultures of $B$. diphtheriae and $B$. pseudodiphthericus.

I. Routine study.-Stain B. diphtheriae with Loeffler's methylene-blue instead of gentian-violet. The staining may be facilitated by the application of mild heat. Observe particularly the peculiar effects of the staining process, and make accurate sketches of what yon see under the microscope.

2. Special study $A$.-Neisser's method of staining to demonstrate the granules in the bacilli:

a) Prepare two solutions.

\section{SOLUTION I}

Methylene-blue....................

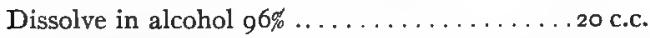

Add distilled water... . . . . . . . . . . 950 c.c.

Glacial acetic acid....................50 c.c.

SOLUTION II

Bismarck brown....................

Distilled water................... rooo c.c.

b) Prepare a film in the usual manner from a culture of $B$. diphtheriae on blood-serum (furnished).

c) Stain in Solution I for 5 to 6 seconds.

d) Wash in water. 


e) Counterstain with Solution II for ro-I 5 seconds.

f) Wash in water, examine in water, dry, and mount in balsam.

3. Special study B.-Test for acid formation in a culture, one week old, in neutral glucose-broth by addition of a few drops of litmus-solution.

4. Special study C.-Cultivation of $B$. diphtheriae on eggs. (Method of Wyatt Johnston).

NoTE.-This method is recommended as an emergency culture test, the egg taking the place of Loeffler's blood-serum.

a) Sterilize over the flame a small empty pot of meatextract, or any other vessel of suitable size.

b) Carefully break with sterile forceps the shell of a hard-boiled egg at the blunt end, taking care not to rupture the membrane lining the shell.

c) Flame the exposed part, and carefully free the coagulated albumen from any membrane.

d) Inoculate by gently rubbing some culture or throat swab on the exposed egg-albumen.

$e$ ) Invert and set in the sterilized pot.

f) Place in the thermostat, and

g) Observe the appearance and make a stained preparation after 24 hours.

5. Special study D.-Study of B. xerosis. Obtain mucus from inner angle of the eyelids by stroking with a platinum loop. Make two film preparations, stain one by Gram's method and the other with methylene-blue. Try culture on slant-agar and if impure, plate out. Wherein does $B$. xerosis differ from the other members of the group?

6. Special study E.- 
EXPERIMENT I

a) Procure a guinea-pig (or rabbit), and clip the hair over a small area on the surface of the abdomen.

b) Cut a small opening in the skin, and separate the skin from the muscles below by pushing in sterile scissors. Expand these slightly and after closing again remove. This forms a small pocket.

c) Carry I loopful of a 24-hour-old agar-culture into this pocket.

\section{EXPERIMENT II}

a) Heat a 24 -hour-old broth-culture in the waterbath for 30 minutes at $60^{\circ}$.

b) Inject 0.25 c.c. of this heated culture subcutaneously into another guinea-pig (or rabbit)

c) Observe and compare in both animals the results by taking note of the ante-mortem phenomena and the lesions post-mortem. What difference is there in the activities of the two cultures inoculated, and to what are these differences due? 




\section{CHAPTER XVI}

\section{THE HEMORRHAGIC SEPTICEMIA GROUP}

Members-

Bacillus pestis (bacillus of bubonic plague).

B. cuniculicida (bacillus of fowl-cholera, bacillus of rabbit septicemia, Bacillus der Rinderseuche, Bacillus der Schroeineseuche, etc.).

REFERENCES-

Textbook: Moore, The Pathology of Infectious Diseases of Animals.

Inoculate agar-slants from stock-culture of $B$. cuniculicida. For obvious reasons, B. pestis will not be studied culturally.

I. Routine study.-Stain with Loeffler's methyleneblue and anilin-gentian-violet. Observe "polar staining." What is "polar staining"?

2. Special study.-Inoculation of rabbit subcutaneously or by scarification. When dead, study in the usual manner, and observe particularly the hemorrhages produced in the serous membranes. Make cultures from heart's blood, where large numbers of bacilli will be found. Also make a stained preparation, and note the typical polar staining. 


\section{CHAPTER XVII \\ THE ANTHRAX GROUP}

Members-

Bacillus anthracis.

B. subtilis, including several varieties.

Great caution is necessary in handling $B$. anthracis.

Inoculate agar-slants from stock-cultures of $B$. anthracis and $B$. subtilis.

I. Routine study.-Observe particularly the colonies formed on agar and gelatin plates.

2. Special study A.-Make "impression preparation" (Klatschpräparat) from a surface colony on a gelatinplate.

Method-

a) Clean and flame a cover-slip carefully.

b) Place on a colony of suitable size, and gently press down, taking care not to press so hard as to disturb the characteristic shape of the colony.

c) Lift the cover-slip carefully with the forceps.

d) Dry, fix, and stain with methylene-blue or by Gram's method.

e) Examine under low and high power (dry lens), and sketch what you see.

3. Special study B.-Staining of spores. Read carefully the different methods of staining spores. Why are special stains necessary?

Moeller's method:

a) Prepare several (five or six) films in the usual manner from 24-hour-old agar-cultures of $B$. anthracis (or B. subtilis). 


b) Place in chloroform for 2 minutes.

c) After drying in the air, cover with a 5 per cent. solution of chromic acid for 2 minutes.

d) Wash thoroughly in water.

e) Cover with carbol-fuchsin and heat for 5 minutes over the water-bath at $100^{\circ}$, or over a small flame, simmering gently all the while.

f) Decolorize with I per cent. sulphuric acid for $25-$ 30 seconds.

g) Wash thoroughly in water.

h) Mount in water, and examine under the microscope to see if spores are cherry-red and the protoplasm colorless or faintly pink.

i) Counterstain with methylene-blue for $\operatorname{to-x} 5$ seconds without heat.

j) Wash, examine in water, and then mount in balsam.

Note.-The body of the cell should appear blue; the spore, red.

4. Special study C.-Demonstration of filamentformation.

a) Spread a loopful of a broth-culture of $B$. anthracis or $B$. subtilis on a clean cover-glass.

b) Dry and fix in the flame.

c) Cover with strong acetic acid (80 per cent.) for 5-ro seconds.

d) Wash in water.

e) Stain with gentian-violet.

f) Examine in water, dry, and mount in balsam. What is the object of applying acetic acid?

5) Special study D.-Inoculate a guinea-pig subcutaneously with 0.2 c.c. of a 24 -hour-old broth-culture of $B$. anthracis, or insert a loopful of a 24 -hour-old agar- 
culture in a "pocket" under the skin. When the animal is dead, perform an autopsy, and observe particularly the hemorrhagic and gelatinous edema under the skin; also the enlarged spleen and the hemorrhagic adrenals. Make a stained preparation from the heart's blood, and observe the lack of spores, and also the presence of capsules and degenerate forms, which do not stain. What prevents the formation of spores? How would you distinguish $B$. anthracis from $B$. subtilis? 




\section{CHAPTER XVIII \\ THE SPIRILLUM GROUP}

MEMBERS-

Spirillum cholerae asiaticae.

$S p$. of Finkler and Prior.

Sp. Metchnikovi.

Sp. tyrogenum.

And a number of spirilla indigenous to water.

Great caution must be exercised in manipulating the spirillum of asiatic cholera.

Inoculate agar-slants from stock-cultures of $S p$. cholerae asiaticae, Sp. of Finkler and Prior, and $S p$. Metchnikovi.

I. Routine study.-In addition to the usual media, inoculate an extra tube of Dunham's peptone-solution from each organism. Observe carefully from day to day the action of these three organisms on gelatin, and compare the results by tabulation. Observe the formation of coccoid involution forms on agar after 3 days. Also make plates in gelatin, observe the colonies from day to day, and compare.

2. Special study A.-Test for the nitroso-indol or cholera-red reaction. (See test for indol, p. 83.) Make two tests, using one of the cultures in Dunham's solution after 24 hours, the other after 6 days. Compare the results of these two tests.

3. Special study B.-Stain for flagella by Loeffler's method (see p. 86).

4. Special study C.-Schottelius' enriching method. 
a) Make a solution of I g. Witte's peptone in roo c.c. of water.

b) Distribute in three small Erlenmeyer flasks, and sterilize in autoclav.

c) Inoculate one of these flasks with Sp. chol. asiat. and $B$. chol. suis or any other motile bacillus.

d) Incubate at $37^{\circ}$ for $18-24$ hours. Make a stained preparation from the surface of the liquid.

e) After that time, take one loopful from the surface, inoculate the second flask, and incubate as before.

f) After 18-24 hours, make a stained preparation from the surface of the second flask, and examine for spirilla.

g) Transfer a loopful from the surface of the second flask to the third one, and incubate as before.

h) After 18-24 hours, again stain and examine for spirilla. By this time usually a film has formed which contains the spirilla in practically pure culture. How is this phenomenon explained, and what is its.epidemiological value?

5. Special study $D$.-Inoculate a pigeon intramuscularly with 0.5 c.c. of a broth-culture of Sp. Metchnikovi. The breast of the pigeon is laid bare, washed with mercuric chlorid and alcohol, and the syringe is plunged into the muscle-fibers and discharged. After death, note the peculiar appearance, resembling that of boiled beef. Make stained preparations from blood and muscle-juice, and examine for spirilla. 




\section{CHAPTER XIX}

THE GROUP OF ACID-RESISTING BACILLI

MEMBERS-

Bacillus tuberculosis.

B. leprae.

B. smegmae.

Moeller's grass bacilli, including a number of bacilli found on grass, dung, in butter, milk, etc.

For obvious reasons, $B$. tuberculosis will not be studied culturally. For comparison, the culture characteristics of Moeller's grass bacillus are instructive.

Inoculate an agar-slant from a stock-culture of Moeller's grass bacillus.

I. Routine study.

2. Special study A.-Method of staining acid-resisting bacilli.

a) Pick out purulent matter from the sputum ot a tuberculous patient and spread carefully on a coverglass.

b) Dry and fix as usual.

c) Heat for one minute on a water-bath at $100^{\circ}$, or over a small flame with carbol-fuchsin.

d) Decolorize with acid alcohol (2 per cent. $\mathrm{HCl}$ in 80 per cent. alcohol) until the film in its thin parts has lost almost all its color.

e) Counterstain with methylene-blue for ro seconds (cold).

f) Examine and mount in balsam.

NoTE.-Make a second preparation, substituting anilingentian-violet for carbol-fuchsin, and Bismarck brown for methylene-blue. 
3. Special study B.-Observe the lesions in a guinea-pig or rabbit dead of tuberculosis, which has been inoculated about four weeks previously. Note the caseous matter at the site of the inoculation, the enlarged yellowish inguinal and axillary glands, the small gray or yellowish tubercles in the liver, lung, spleen, mesentery, etc. Stain for bacilli from such sites. Make cultures on glycerin-agar or dog-serum (see the textbook).

4. Special study C.-Stain Moeller's grass bacillus from agar-culture by the same method, omitting the counterstain.

5. Special study D.-

a) Grind in a mortar, previously sterilized, a small amount of a culture of the grass bacillus, and mix with some sterile milk.

b) Make a cover-slip preparation of this mixture, and stain for tubercle bacilli. At what conclusions as to the quality of the milk would you arrive, if you should find acid-resisting bacilli in a fresh sample of milk? 




\section{CHAPTER XX}

\section{MISCELLANEOUS BACTERIA}

Inoculate agar-slants from stock-cultures of Bacillus mallei and Micrococcus melitensis. Exercise great caution in handling $B$. mallei.

I. Routine study.-Note especially the growth of B. mallei on potato. Also note the morphology of Micr. melitensis and the staining properties of $B$. mallei. Is $B$. mallei motile?

2. Special study.-Intraperitoneal injection of $B$. mallei from a broth-culture into a male guinea-pig. Why do you select a male guinea-pig? What is Straus's method of diagnosis of glanders? 


\section{CHAPTER XXI \\ THE ACTINOMYCES GROUP}

Inoculate agar-slants from Actinomyces bovis (hominis) and Actin. asteroides.

\section{REFERENCES-}

Stokes, American Journal of the Medical Sciences, November, I904.

Wright, Jour. of Med. Research, May, I905.

I. Special study A.-Transfer from agar-slants to broth and potato only, and make descriptions and stained preparations as usual.

2. Special study B.-Suspend a small amount of the potato-culture in a physiological salt solution, and examine under the low power.

3. Special study C.-

a) Examine a sample of actinomycotic tissue (bovine) in the fresh state, for so-called "sulphur granules." Crush some in salt solution under a cover-slip and search for "clubs," using the low and high power dry lenses.

b) Crush others, dry, fix, and stain by Gram, counterstain with eosin or Bismarck brown.

c) Write a full description of the biology of the organism, illustrated by drawings.

d) What other actinomycetes have been described as pathogenic? Give their names and the diseases which they produce. 




\section{CHAPTER XXII \\ THE ANAËROBIC GROUP OF BACILLI}

Members-

Bacillus tetani.

B. oedematis maligni.

$B$. aërogenes capsulatus.

B. anthracis symplomatici.

B. botulinus.

And others.

Study the different methods of anaërobic cultivation in the textbook.

I. Special study A.-Park's method.

a) Boil three tubes of glucose-agar vigorously for 5 minutes, to drive out the dissolved oxygen. (Why is the presence of glucose desirable?)

b) Cool to $43^{\circ}$ and inoculate from stock-culture of $B$. tetani, B. oedematis maligni, and $B$. aërogenes capsulatus.

c) Solidify rapidly by immersion in cold water.

d) Cover the medium with a thin layer of liquid paraffin.

e) Place in thermostat.

NoTE.-The layer of parraffin effectively excludes the atmospheric oxygen, which would be inhibitory to the growth of the anaërobes. The oxygen necessary for their multiplication is derived from nutrient material of the medium.

2. Special study B.-Wright's modification of Buchner's method.

a) Liquefy, as before, six glucose-agar tubes, the plugs of which have been replaced by absorbent cotton. 
Cool three to $43^{\circ}$, and inoculate while fluid. Let the other three become solid, and make stab-cultures.

b) Sterilize the cotton stoppers in a flame, and with the forceps, sterilized in a flame, push the stoppers into the test-tubes for the distance of about $\mathrm{I}$ inch $(2-3 \mathrm{~cm}$.).

c) Pour into the tubes (upon the cotton stoppers) 2 c.c. of a saturated solution of pyrogallic acid in water, followed by 2 c.c. of a 2 per cent. solution of $\mathrm{NaOH}$.

d) Cork the tubes immediately with rubber stoppers, and keep upside down.

e) Incubate at the required temperature.

Upon what principle does this method depend? Do the organisms grow both aërobically and anaërobically?

When you have obtained a growth, stain the organisms with anilin-gentian-violet and by Gram's method. Describe and illustrate.

3. Special study C.-Cultivation by Buchner's method, using fruit-jars.

a) Into a Mason fruit-jar of ordinary type deposit Io g. of pyrogallic acid.

b) Smear vaselin around the mouth of the jar.

c) Pour into the jar roo c.c. of a I per cent. solution of $\mathrm{NaOH}$.

d) Then deposit in the jar culture-tubes previously inoculated.

$e$ ) Tightly fasten the cover of the jar, and incubate at $37^{\circ}$ for $48-72$ hours.

4. Special study D.-Cultivation in hydrogen gas.

a) Inoculate all media from stock-cultures obtained.

b) Fit up apparatus as shown in Fig. 34 .

c) Place culture-tubes in a Novy jar (Fig. $34, a$ ). 


d) Open the faucet $(b)$ of the gas-generator $(c)$, containing zinc and hydrochloric acid. The hydrogen gas generated passes through two jars, one of which contains concentrated sulphuric acid $(d)$, the other a to per cent. solution of sodium hydrate (e). Gradually the Novy jar is filled with hydrogen gas, which can be tested by holding a culture-tube over the opening $(f)$, and then over a burning match or gas flame. As long as any detonation takes place the hydrogen is still mixed with

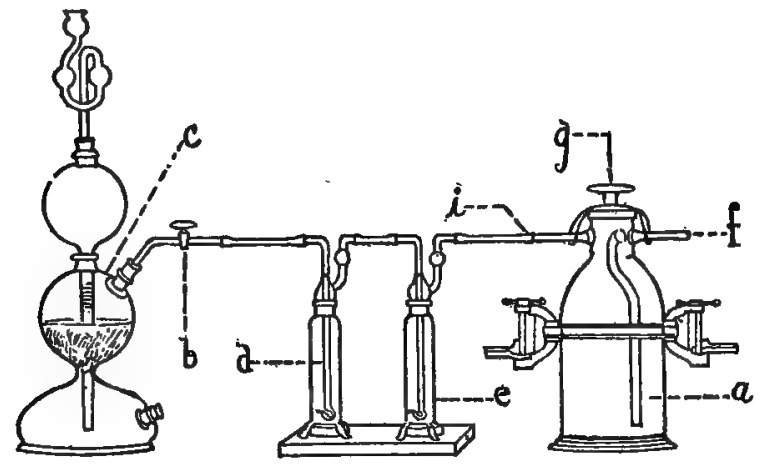

Fig. 34

Anaërobic Cultivation in Hydrogen Gas
a. Novy jar
b. Glass cock
c. Gas generator
d. Sulphuric acid

e. Sodium hydrate solution

f. Opening of Novy jar

g. Stopper

atmospheric oxygen. When finally the hydrogen in the Novy jar is pure, close it off by turning the stoppers $(g)$ and $(b)$, and place it in the incubator. The whole process occupies about to or 15 minutes.

5. Special study C.-Inoculation of a rabbit with $B$. aërogenes capsulatus.

a) Shave the ear of the rabbit. 


\section{IIO LABORATORY GUIDE IN BACTERIOLOGY}

b) Wash with mercuric chlorid solution and alcohol.

c) Inoculate intravenously with 0.5 c.c. of a 24 -hourold milk-culture.

d) After the culture has been fully distributed in the circulation, which takes at the most 3 minutes, kill the rabbit by a quick blow on the back of the neck.

e) Put the rabbit in a warm place-say, on top of the thermostat-for I 8 hours, or from 6 to 8 hours inside of the thermostat.

f) After this time has elapsed, perform an autopsy. Note the crackling, on pressure, over the axillary or inguinal regions. The rabbit is swollen to a great extent. Skin the animal carefully, without opening the abdominal cavity; then quickly puncture the abdominal wall and bring a flame to the opening. Note that the escaping gas will burn with a blue flame. What is this gas? Also note the disorganized condition of the liver, spleen, and kidney.

g) Make capsule stains from the heart's blood or organs by Welch's method (modified). The modification of Welch's method is as follows: Proceed in the manner indicated on p. 77 and, after washing the acetic acid off with the stain (carbol-fuchsin or gentian-violet), dry with filter paper, heat the specimen for 5 to ro seconds before washing off with the salt solution. Then proceed as before.

6. Special study F.--Staining of spores of B. tetani and $B$. oedematis maligni from 3 -day-old glucose-agarcultures (see p. 98).

7. Special Study G.-Inoculation of a white mouse or guinea-pig with B. letani or its toxin (O.OI c.c.) in the hind-leg or over the root of the tail (if a mouse). Note 


daily the condition of the animal, and when dead make cultures and cover-slip preparations from the site of the inoculation.

8. Special study H.-Inoculate a white rat with garden earth subcutaneously or in a pocket above root of tail. Note the condition of the animal daily. When dead, make cultures and cover-slip preparations from the site of the inoculation. Would you expect to find bacilli in the heart's blood or organs? Give reason for your answer. 


\section{CHAPTER XXIII}

\section{ISOLATION OF UNKNOWN BACTERIA FROM A}

MIXTURE

I. Make hanging-drop, stained, and Gram preparations from the mixture. Note observations and results.

2. Melt five or six agar-tubes, and cool to $43^{\circ}$.

3. Transfer 5 or 6 loopfuls of the mixture to a tube of liquid agar, from this to a second, and so on until all the melted tubes are inoculated.

4. Pour into sterile Petri dishes, and mark them with successive numbers and the date. Place in the thermostat.

5. After 24 hours examine the colonies under the low power, describe them in the usual manner, and transfer to agar-slants all those which show different appearances.

6. Now proceed with the usual routine study. Make hanging-drop, stained, and Gram preparations, transfer to all the media, and describe the culture characteristics accurately. Make sketches in the usual manner.

7. Special tests may become necessary after 24 or 48 hours. Such tests may consist of--

Capsule stain.

Spore stain.

Stain for acid-resisting bacilli.

Fermentation tests of all those which produce gas in glucose-agar. Also note the growth, or lack of growth, in the closed arm of the fermentation-tube.

Test for acid in neutral broth. 


ISOLATION OF UNKNOWN BACTERIA II3

Test for agglutination.

Test for indol.

Anaërobic cultivation.

Inoculation of animals.

For final diagnosis consult your notes and textbook. 


\section{CHAPTER XXIV}

BACTERIOLOGICAL EXAMINATION OF WATER, AIR AND MILK

EXERCISE I. BACTERIOLOGICAL ANALYSIS OF WATER REFERENCES-

Horrocks, Bacteriological Examination of Water.

Prescott and Winslow, Elements of Water Bacteriology.

Fuller and Johnston, Journal of Experimental Medicine, Vol. IV.

Committee Report of the American Public Health Association, Jour. of Infectious Diseases, Suppl. I, May, I905.

A bacteriological examination of water is made for the purpose of determining-

I. Bacterial numbers.

2. Bacterial species.

3. Sewage contamination.

Collection of samples.-Procure wide-mouthed, glassstoppered bottles, having a capacity of at least roo c.c. After careful cleaning and drying, wrap them in lead-foil, and sterilize in the hot-air oven for I hour at $160^{\circ}$; then deposit them in a metal or wooden case. The samples from surface waters should be taken at least one foot below the surface, to avoid contamination with organisms from the air. If possible, samples should be plated on the spot or in the laboratory within an hour at the very latest. But when a greater interval of time must occur, the samples should be taken to the laboratory packed in ice, despite the probability of thus destroying a certain percentage of the bacterial flora.

Method of examination.-A number of pipettes of various sizes (I c.c., 2 c.c., 5 c.c., and to c.c.) are plugged 


with cotton and sterilized in the hot-air oven. Then a number of Erlenmeyer flasks are filled with ror c.c. of distilled water, and these are sterilized in the autoclav at $\mathrm{I} 20^{\circ}$ for 5 minutes. About I c.c. of water is lost by evaporation during this process, so that the sterile flasks contain Ioo c.c. each. ${ }^{\mathrm{I}}$

\section{Method of procedure.-}

I. With a sterile pipette remove I c.c. of water from one of the sterilized dilution flasks.

2. Carry over to this flask I c.c. of the sample after a thorough shaking. The dilution is now I: Ioo. Mark with glass pencil.

3. With a sterile to c.c. pipette remove to c.c. from another dilution flask, and add to the remainder Io c.c. of the first dilution. We now have a dilution of $\mathrm{I}: \mathrm{I000}$. (See dilution table; p. I r 7.) Make a number of dilutions in this manner, carrying the dilutions higher in proportion to the quality of the water to be examined.

4. Melt a number of agar and gelatin tubes, corresponding to the number of dilutions made, and cool to $43 .^{\circ}$ Read in reference book carefully the chapters on the importance of using both agar and gelatin media.

5. Mix carefully I c.c. of each dilution flask with a tube of liquified medium, respectively.

6. Pour into Petri dishes.

7. Incubate the agar-plates at $37^{\circ}$, and keep the gelatin-plates at room temperature. The mixing of the diluted sample and agar may also be effected by pouring the sample into a Petri dish and mixing it with the liquid medium. This method is not as convenient, but slightly

"If work is carried on with dilutions of 1 : 100 only, the flask may conveniently be filled with 100 c.c. water instead of ror c.c. It is then not necessary to remove I c.c. of water, as only 09 c.c. are left after sterilization. 


\section{II6 LABORATORY GUIDE IN BACTERIOLOGY}

more accurate, as no loss takes place by adhesion to the tube.

Estimation of colonies.-The colonies are then counted after 48 hours, by means of a colony-counter (Fig. 35). Plates should be counted which contain no more than 200-300 colonies. If it is necessary to count plates with a large number of colonies, an estimate must be made by counting different sections of the plate-counter and averaging the result for the whole plate.

Species determination.-If the different species of bacteria are to be studied, the colonies must be examined by the naked eye and the low power. Then those which appear to be different are transferred to slant-agar tubes, and from these to the ordinary media.

Sewage contamination.-The presence of Bacillus coli and streptococci is sufficient indication of sewage contamination in water.

Method of examination for B. coli and streplococci.--

I. I c.c. of the sample, or, if necessary, of the diluted sample, is added to a series of ten fermentation-tubes, containing sterile 2 per cent. glucose-broth.

2. Place in thermostat.

3. Examine after 12-I 8 hours.

4. Examine a loopful of the sediment in a stained preparation.

Example.-If I c.c. of the sample is added to each fermentation-tube, and six show gas-formation, there would be six colon bacilli in each Io c.c. if undiluted water is employed. By this method fairly accurate results may be obtained.

Isolation is accomplished by plating in an agar medium containing 2 per cent. lactose and ro per cent. litmus solution. 


EXAMINATION OF WATER, AIR, AND MILK II7 DILUTION TABLES FOR AGGLUTINATION

\begin{tabular}{l|c|c|c}
\hline \multicolumn{1}{c|}{ Number } & Amount of Serum & $\begin{array}{c}\text { Amount of Salt } \\
\text { Solution }\end{array}$ & Final Dilution \\
\hline I........ & I part & 9 parts & I: I0 \\
$2 \ldots \ldots \ldots$ & I part of No. I & 9 parts & I: 100 \\
$3 \ldots \ldots \ldots$ & I part of No. 2 & 9 parts & I: 1000 \\
$4 \ldots \ldots \ldots$ & I part of No. 3 & 9 parts & I: 10000 \\
\hline
\end{tabular}

\begin{tabular}{|c|c|c|c|c|}
\hline \multicolumn{3}{|c|}{$\begin{array}{l}\text { Amount of Serum or } \\
\text { Serum Dilution }\end{array}$} & Suspension & Final Dilution \\
\hline \multicolumn{3}{|c|}{2 part clear serum..... } & I8 parts & I : IO \\
\hline I & " $" 6$ & " $\quad \ldots \ldots$ & rg" & $I: 20$ \\
\hline & parts dil., & No. I..... & I 5 & $I: 40$ \\
\hline & $4 \$$ & No. $1 . . .$. & 16 " & $I: 50$ \\
\hline 2.5 & $5 “$ & No. I..... & I $7 \cdot 5^{\prime \prime}$ & I : 80 \\
\hline & " $"$ & No. I.... & & I : IQO \\
\hline $\mathbf{I}$ & " " & No. $1 \ldots$. & I9 & $I: 200$ \\
\hline 4 & " $"$ " & No. $2 \ldots$. & 16 " & $I: 500$ \\
\hline 2 & " $"$ & No. $2 \ldots .$. & I8 " & $I: I 000$ \\
\hline I & " " & No. $2 \ldots .$. & I9 & I : 2000 \\
\hline 4 & " at & No. $3 \ldots$. & I6" & I : 5000 \\
\hline 2 & " a & No. $3 \ldots$. & I8 " & I : I0000 \\
\hline I & $"$ & No. $3 \ldots$ & I9 & I : 20000 \\
\hline 4 & “ & No. $4 \ldots$. & I6 & $1: 50000$ \\
\hline 2 & " & No. $4 \ldots$. & I8 " & I : I00000 \\
\hline I & " $\quad$ is & No. $4 \ldots$. & 19" & I : 200000 \\
\hline
\end{tabular}

DILUTION TABLE FOR WATER OR MILK ANALYSIS

\begin{tabular}{|c|c|c|c|}
\hline Number & Amount of Dilution & $\begin{array}{l}\text { Amount of } \\
\text { Sterile Water }\end{array}$ & Final Dilution \\
\hline $\begin{array}{l}1 \ldots \ldots \\
2 \ldots \ldots \\
3 \ldots \ldots \\
4 \ldots \ldots \\
5 \ldots \ldots \\
6 \ldots \ldots \\
7 \ldots \ldots \\
8 \ldots \ldots \\
9 \ldots \ldots \\
10 \ldots \\
11 \ldots \ldots \\
12 \ldots \ldots \\
13 \ldots \ldots\end{array}$ & $\begin{array}{l}\text { Original } \\
\text { Ic.c. of No. I } \\
2 \text { oc.c. of No. } 2 \\
\text { Ioc.c. of No. } 2 \\
5 \text { c.c. of No. } 2 \\
\text { 2c.c. of No. } 2 \\
\text { Ic.c. of No. } 2 \\
5 \text { c.c. of No. } 4 \\
2 \text { c.c. of No. } 4 \\
\text { Ic.c. of No. } 4 \\
5 \text { c.c. of No. } 7 . \\
\text { 2c.c. of No. } 7 . \\
\text { Ic.c. of No. } 7\end{array}$ & $\begin{array}{l}99 \mathrm{c} . \mathrm{c} . \\
8 \mathrm{cc} . \mathrm{c} . \\
90 \mathrm{c} . \mathrm{c} . \\
95 \mathrm{c} . \mathrm{c} . \\
98 \mathrm{c} . \mathrm{c} . \\
99 \mathrm{c} . \mathrm{c} . \\
95 \mathrm{c} . \mathrm{c} . \\
98 \mathrm{c} . \mathrm{c} . \\
99 \mathrm{c} . \mathrm{c} . \\
95 \mathrm{c} . \mathrm{c} . \\
98 \mathrm{c} . \mathrm{c} . \\
99 \mathrm{c} . \mathrm{c} .\end{array}$ & $\begin{array}{l}I: I \\
I: I 00 \\
I: 500 \\
I: I 000 \\
I: 2000 \\
I: 5000 \\
I: I 0000 \\
I: 20000 \\
I: 50000 \\
I: 100000 \\
I: 200000 \\
I: 500000 \\
I: 1000000\end{array}$ \\
\hline
\end{tabular}




\section{II8 LABORATORY GUIDE IN BACTERIOLOGY}

EXERCISE II. BACTERIOLOGICAL EXAMINATION OF AIR

An approximate determination of the number of bacteria in the air can be made by the following simple - method: Place a certain amount (50 c.c.) of broth in an Erlenmeyer flask (Fig. 36, a). This flask is provided with a rubber stopper (b), provided with two holes, through which the two glass tubes ( $c$ with a wide opening

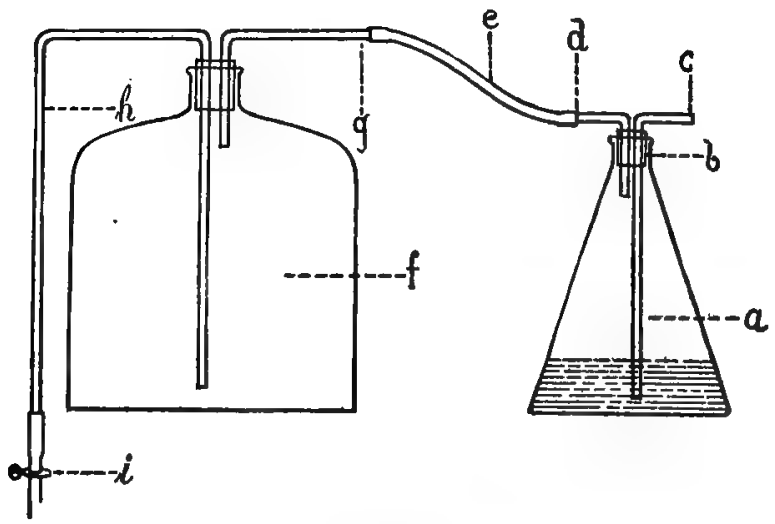

FIG. 36

Apparatus for Determining the Number of Bacteria in a Definite Volume of Air
a. Erlenmeyer flask
b. Rubber stopper $c-d$. Glass tubes

$f$. Five-liter flask
$g-h$. Glass tubes
f. Pinchcock

and $d$ ) lead. Cotton plugs are then inserted at $c$ and $d$, and the apparatus is sterilized in the autoclav. A large bottle $(f)$, containing 5 liters of water, is then provided with a rubber stopper, and also with two glass tubes $(g$ and $h) ; h$ is connected with a short piece of rubber hose and a pinchcock $(i)$. When the Erlen- 


meyer flask and contents are sterilized, the tube $d$ is connected, by means of the rubber hose $e$, with $g$, and the plug at $c$ is removed. By opening the pinchcock $i, 5$ liters of air are aspirated through the broth in flask $a$. The flask is then disconnected, and I. c.c. is plated in agar and I c.c. in gelatin. The former is incubated at $37^{\circ}$, and the latter kept at room temperature. After 48 hours the colonies are counted, and the result is multiplied by 5o. This then represents the amount of bacteria in 5 liters of air.

\section{EXERCISE III. BACTERIOLOGICAL STUDY OF MILK}

The method for determining the number of bacteria in milk is fundamentally the same as for water, except that dilutions must be carried higher, as milk generally contains much larger numbers of bacteria.

Sterilization and pasteurization of milk.-Some of the important germs in milk are saprophytes (which under favorable circumstances produce disagreeable odors or tastes), and such pathogens as the bacillus of tuberculosis (which may be derived from the cow, or may be an accidental contamination), the typhoid bacillus, the bacillus of "summer complaint" in children (possibly identical with the bacillus of epidemic dysentery), the germs of cholera, diphtheria, and scarlet fever. All these, except B. tuberculosis, flourish in milk at its ordinary temperature.

None of the methods employed in sterilizing milk render it sterile in the bacteriological sense of the word, but by means commonly employed most of the nonsporing pathogenic bacteria are destroyed, along with a large number of saprophytes, thus rendering the milk 
comparatively safe and less subject to the ordinary fermentative changes.

r. Sterilization at $100^{\circ}$ for 30 minutes.-Such milk, if chilled and kept at a low temperature, will remain unchanged for more than a week; but, by the heating, certain alterations have been produced in its taste and nutritive qualities which render it supposedly less fit for food than when pasteurization is employed.

2. Pasteurizing milk.-The changes occurring in milk, as above mentioned, begin at about $82^{\circ}$. Sterilization at a low temperature is accomplished by raising the temperature to only $75^{\circ}$ for a period of 20 minutes. This has been shown to be sufficient to kill the germs of typhoid, cholera, diphtheria, and pyogenic cocci. Spored organisms are not killed. As shown by Theobald Smith, tubercle bacilli, when suspended in distilled water, physiological salt solution, broth, and milk, are destroyed at $60^{\circ}$ in $15^{-20}$ minutes; but, if milk containing tubercle bacilli has its surface exposed to the air when heated to $60^{\circ}$, the pellicle which forms on its surface may contain living tubercle bacilli after an exposure of 60 minutes.

Study of the effect of the above two methods of sterilization as compared with each other and with unsterilized milk:

I. From the fresh milk provided make three agarplates, using $\mathrm{r}, 2$, and 3 loopfuls, respectively.

2. Fill about ro c.c. into each of ten sterile culturetubes, and keep one at room temperature and one in the thermostat.

3. Treat four of these tubes in the following manner: Place water in a saucepan sufficient to cover completely 


the milk when the tubes are immersed in it. Raise the temperature to $75^{\circ}$, and keep it there by regulating the flame. The tubes of milk are then immersed in the water, and kept there for 30 minutes, as it requires about Io minutes for the milk in the tubes to reach the temperature of the water. The tubes are then taken out and cooled quickly by standing them in cold water. Place one of the tubes at incubator and the other at room temperature. Aërate the other two by shaking vigorously for $\mathrm{I} \frac{1}{2}$ minutes. Keep one of these at room temperature, the other in the thermostat.

4. Place two more milk-tubes in the Arnold at $100^{\circ}$ for 30 minutes. Keep one at room temperature and one in the thermostat.

5. The remaining two tubes autoclav at $\mathrm{I} 20^{\circ}$ for 5 minutes, and place one in the thermostat and keep the other at room temperature.

6. Note the conditions of these ten tubes after 2 or 3 days. Compare the results, and tabulate them. Note especially coagulation, time elapsed before coagulation sets in, gas-formation, condition of whey, film, and odor. How are these differences explained?

Plates in lactose-litmus-gelatin should be made from each of these tubes, and the colonies studied and counted. Subcultures on agar-slants may also be made, and the usual media inoculated from these, if the individual species are to be studied. 


\section{CHAPTER XXV}

INFLUENCE OF DISINFECTANTS ON THE GROWTH OF MICRO-ORGANISMS

EXERCISE I

I. Prepare fifty-seven Hill's test-rods. These are prepared in the following manner: Glass rods about two inches longer than ordinary culture-tubes are marked with hydrofluoric acid by a circle exactly one inch from the end. A wad of cotton is then wrapped around the middle of the rod, and this is inserted in a culture-tube. The rod is then pushed down until it nearly reaches the bottom. That part of the rod which is free at the upper end is used for labeling. The whole apparatus is then sterilized in the dry-air oven.

2. Fill two wide-mouthed flasks, one with 100 c.c. of a 5 per cent. solution of carbolic acid, the other with Ioo c.c. of a I per cent. solution.

3. Fill two similar flasks, one with roo c.c. of a solution of mercuric chlorid $\mathrm{I}: \mathrm{s}, 00$, the other with a solution of $\mathrm{I}: \mathrm{I0}, 000$.

4. Fill two similar flasks, one with Ioo c.c. of a to per cent. solution of formalin ( 40 per cent. formaldehyde), the other with a I per cent. solution.

5. Prepare 48-hour broth-cultures of Staphylococcus pyogenes aureus, Bacillus coli and B. typhosus from stock-cultures.

6. Dip nineteen of these rods into each of these cultures respectively, to the depth of one inch; set them aside in their tubes to dry over night in the thermostat, after marking each tube carefully. 


EXERCISE II

We have now six flasks containing different solutions of disinfectants.

I. Place in each one of these flasks nine of the prepared rods, three of which have been dipped in the Staph. pyogenes aureus culture, three in the $B$. coli culture, and three in the B. typhosus culture.

2. Take three rods (one of each organism) out of each flask after the lapse of half a minute, wash by gently pouring sterile physiological salt solution over them into a dish containing mercuric chlorid solution I : Iooo, and place each rod in a tube of sterile broth

3. Repeat the proceedings of step 2 with a second series of rods after 2 minutes.

4. Repeat again after 5 minutes with the remaining series.

5. Place all tubes (fifty-seven) in the thermostat. Three of these tubes have not been dipped into any one of the six flasks containing antiseptics, and are incubated with the others as controls.

6. Observe the results carefully on each of the four successive days, and on the last day prove the relative growth by making agar-plates with I c.c. of each culture, and count the colonies after 24 hours.

7. Tabulate the results, and state your conclusions.

EXERCISF III. INFLUENCE OF SUNLIGHT EXPERIMENT I

I. Inoculate a flask containing roo c.c. of sterile water with $B$. coli.

2. After thoroughly shaking, take I c.c. by means of a sterile pipette, and plate in agar. Place the plate in the thermostat. 
3. Expose the flask to sunlight for several hours.

4. Make another plate with I c.c. of the suspension, and place in a thermostat.

5. After 48 hours count both plates, and compare the results.

\section{EXPERIMENT II}

I. Melt a tube of agar and cool to $43^{\circ}$.

2. Inoculate with $B$. coli (or any other organism).

3. Pour into a sterile Petri dish.

4. After solidification, turn bottom side up, and paste a strip of black paper on the glass, covering part of the surface.

5. Expose to direct sunlight for several hours, and note the result.

EXERCISE IV. INFLUENCE OF MOIST HEAT

Read the methods of determining the thermal deathpoint of bacteria in the textbook.

I. Prepare six broth-cultures each of $B$. coli and $B$. subtilis.

2. Place four cultures of each organism in the waterbath and heat.

3. Remove one of each at $40^{\circ}$, one of each at $60^{\circ}$, one of each at $80^{\circ}$, and keep one of each for Io minutes at $100^{\circ}$.

4. Place one tube of each organism in the autoclav, and heat to $120^{\circ}$ for 5 minutes.

5. Now place all twelve tubes in the thermostat, including one of each organism as a control.

6. After 24 hours, make plates of each tube in agar, and place them in the thermostat.

7. After 24 hours, count the colonies and compare the results. 




\section{APPENDIX I}

\section{SPECIAL MEDIA}

STANDARD METHOD OF PREPARING BROTH, NUTRIENT GELATIN AND NUTRIENT AGAR

(Report of American Public Health Assocciation,Vol. XXX, I905.)

\begin{tabular}{|c|c|c|}
\hline BROTH & Gelatin & AGAR \\
\hline 1. & & $\begin{array}{l}\text { Boil I } 5 \mathrm{~g} \text {. thread agar in } 500 \\
\text { c.c. water for half an hour } \\
\text { and make up weight to } 500 \\
\text { g., or digest for ro minutes } \\
\text { in the autoclav at I } 0^{\circ} \\
\text { Let this cool to about } 60^{\circ}\end{array}$ \\
\hline $\begin{array}{l}\text { 2. Infuse } 500 \mathrm{~g} \text {. lean meat } 24 \\
\text { hours with roo c.c. of dis- } \\
\text { tilled water in refrigerator. }\end{array}$ & Ditto. & $\begin{array}{l}\text { Infuse } 500 \text { g. lean meat } \\
24 \text { hours with } 500 \text { c.c. of } \\
\text { distilled water in refriger- } \\
\text { ator. }\end{array}$ \\
\hline $\begin{array}{l}\text { 3. Make up any loss by evap- } \\
\text { oration. }\end{array}$ & Ditto. & Ditto. \\
\hline $\begin{array}{l}\text { 4. Strain infusion through } \\
\text { cotton flannel. }\end{array}$ & Ditto. & Ditto. \\
\hline 5. Weigh filtered infusion. & Ditto. & Ditto. \\
\hline 6. Add I \% Witte's peptone. & $\begin{array}{l}\text { Ditto. } \\
\text { And Io \% gold la- } \\
\text { bel sheet gelatin. }\end{array}$ & Add $2 \%$ Witte's peptone. \\
\hline $\begin{array}{l}\text { 7. Warm on water-bath stir- } \\
\text { ring till peptone is dis- } \\
\text { solved, and not allowing } \\
\text { the temperature to rise } \\
\text { above } 60^{\circ} \text {. }\end{array}$ & $\begin{array}{l}\text { Warm on water- } \\
\text { bath until pep- } \\
\text { tone and gela- } \\
\text { tin are dis- } \\
\text { solved, not } \\
\text { above } 60^{\circ} \text {. }\end{array}$ & $\begin{array}{l}\text { Warm on waterbath until } \\
\text { peptone is d is solved, } \\
\text { not above } 60^{\circ} \text {. }\end{array}$ \\
\hline 8. & & $\begin{array}{l}\text { To } 500 \mathrm{~g} \text {. of meat infusion } \\
\text { add } 500 \text { c.c. of the } 3 \% \\
\text { agar, keeping the tem- } \\
\text { perature below } 60^{\circ} \text {. }\end{array}$ \\
\hline
\end{tabular}

9. Heat over boiling water (or steam) for 30 minutes.

Io. Restore loss by evaporation.

II. Titrate after boiling one minute to expel carbonic acid.

12. Adjust reaction to $+1.0 \%$ by adding normal hydrochloric acid or sodium hydrate as required. 


\section{LABORATORY GUIDE IN BACTERIOLOGY}

13. Boil 2 minutes over free flame, constantly stirring.

I4. Make up loss by evaporation.

15. Filter through absorbent cotton and cotton flannel, passing the filtrate through the filter until clear.

r6. Titrate and record final reaction.

17. Tube, using ro c.c. in each tube in the case of gelatin and agar.

18. Sterilize 5 minutes in the autoclav at $120^{\circ}$, or for 30 minutes in streaming steam on 3 successive days. Put at once into ice-water till solidified.

Ig. Store in the ice-chest in a moist atmosphere to prevent evaporation.

\section{Blood-serum -}

I. Fresh ox blood collected in sterile jars (museum jars)

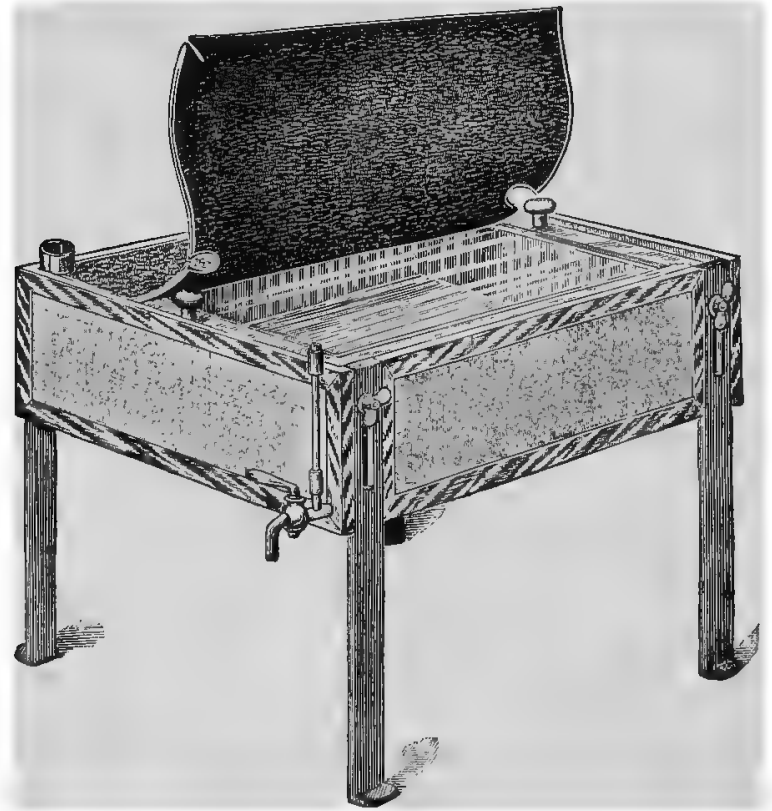

FIG. 37

Koch Inspissator

is set in the ice-chest until the serum has separated. The serum is filtered if necessary.

2. Take 3 parts of this freshly prepared serum and I part of broth containing $\mathbf{I} .5$ per cent. glucose, and mix. 


3. Tube in the usual manner, and place in the Koch inspissator (Fig. 37) two or three rows deep.

4. Incline the inspissator to the proper angle, so as to produce a large sloping surface of the serum.

5. See that the water-jacket contains sufficient water. Place a Bunsen burner below, slowly heat the water to the boiling-point, and boil for 5 minutes. Then turn out the gas.

6. Repeat this process upon the two following days at the same hour.

Note.-Always place about 25 c.c. of water in the inspissator along with the tubes, so as to keep up a saturation of the air in the apparatus. By doing this, one avoids giving the serum a dry surface. See that the inspissator contains a rack made of wooden or metal strips, which keeps the tubes from touching the bottom and wall; otherwise the serum will be overheated forming bubbles in the mass.

Litmus-solution.-Dissolve I part of Merck's pure extract of litmus in 100 parts of water, filter through paper, and sterilize.

Glycerin-broth.-Add 6 per cent. of pure glycerin to ordinary broth.

Glucose-gelatin.-Add I per cent. glucose to ordinary gelatin.

Litmus-lactose-agar (for plating).-Add I per cent. of lactose to sugar-free agar, distribute 8 c.c. in culture-tubes, and add I c.c. of sterile litmus-solution to each tube before using.

Litmus-lactose-gelatin (for plating).-Prepare gelatin in the usual manner, using IO-I2 per cent. of gelatin, and dissolve I per cent. of lactose. Distribute 8 c.c. into culturetubes, and add litmus-solution to each tube before using.

Note-If I c.c. of the liquid to be analyzed is added, I4\% of gelatin should be used.

Litmus-mannit-agar.-Prepare like litmus-lactose-agar, substituting $\mathrm{r}$ per cent. mannit in place of lactose.

Neutral-red agar.-Add enough 0.5 per cent. neutral-red 


\section{LABORATORY GUIDE IN BACTERIOLOGY}

solution to nutrient agar containing I per cent. glucose to produce a clear red color without adjusting reaction.

Litmus-broth.-Add litmus-solution to ordinary broth in the proportion of to per cent.

Glycerin-agar.-Add 6 per cent. pure glycerin to nutrient agar.

Beerwort-gelatin-Autoclav beerwort at $120^{\circ}$ for $5 \mathrm{~min}$ utes to precipitate proteids. After cooling, filter, dissolve ro per cent. gelatin, and clarify with white of egg.

Beerwort-agar.-Prepared like beerwort-gelatin, substituting $I \frac{1}{2}$ per cent. agar in the place of gelatin.

Blood-agar.-A drop of blood, obtained with aseptic precautions, is smeared on the surface of slant-agar.

Litmus-whey (Petruschky, modified by Durham).Casein is precipitated from milk with rennet-extract; the whey is neutralized with 4 per cent. citric-acid solution and heated on the water-bath for half an hour. It is then filtered, and litmus-solution is added until a decided blue color is obtained.

Whey-gelatin.-Add Io per cent. gelatin to clarified whey.

Alkaline blood-serum (Lorrain Smith).-Add I-I.5 per cent. of a ro per cent. solution of sodium hydrate to bloodserum, tube, and sterilize in Arnold or in Koch's serum inspissator.

Bread-paste medium.-Bread is cut into slices, dried in the oven, and pulverized; it is then distributed in roo c.c. flasks until the layer on the bottom is half an inch thick. Water is gradually run in to cover the surface of the bread Sterilize in Arnold.

Mac Conkey's bile-salt-agar.-

Nutrient agar.................... roo c.c.

Sodium taurocholate.................... $0.5 \%$

Peptone............................

This is boiled, clarified, and filtered, and then 2 per cent. lactose is added, tubed, and sterilized in the medium. 


Mac Conkey's bile-salt broth.-

Sodium taurocholate................... $0.5 \%$

Peptone..........................\%

Glucose............................. $0.5 \%$

are dissolved in beef-broth by boiling; then filter and add litmus-solution.

\section{Parieti's solution-}

Carbolic acid..................... c.c.

Hydrochloric acid................... c.c.

Water.........................

Elsner's medium.-I pound of sliced potatoes is grated with a liter of water, and the juice expressed. After filtering for 24 hours, add I per cent. peptone, I per cent. potassium iodid, and ro per cent. gelatin.

Glycerinated potato.--Prepare potatoes in the ordinary manner and soak in a 25 per cent. solution of glycerin. Use a glycerin-solution in the bottom of the tube.

Hay-infusion.- - $\mathrm{g}$ g. of chopped hay are macerated with rooo c.c. of water in the water-bath for 3 hours. Filter and sterilize in autoclav for ro minutes at $120^{\circ}$.

Wine-must.-Wine-must is diluted with four times its weight of water. Dissolve 0.5 per cent. ammonium tartrate, macerate in the water-bath for I hour, filter, and sterilize in Arnold for 3 consecutive days.

Winogradsky's solution (for nitric organisms).-

Dissolve in water................ rooo c.c.

Potassium phosphate................ . $00 \mathrm{~g}$.

Magnesium sulphate................50 g.

Calcium chlorid................... o. or g.

Sodium chlorid. .................

Distribute 20 c.c. in flasks, and add to each flask a small amount of magnesium carbonate. Sterilize in Arnold for 3 consecutive days; then add to each flask 2 c.c. of a sterile 2 per cent. solution of ammonium sulphate. 


\section{I30 LABORATORY GUIDE IN BACTERIOLOGY}

Yeast-water.-I liter of washed yeast or I pound of pressed yeast is boiled with 2 liters of water for $\mathbf{I}$ hour. The reaction is made neutral to phenolphthalein, and the solution is filtered until clear, and sterilized in Arnold for 3 consecutive days.

Dextrose-yeast-water.-IO-I5 per cent. dextrose is dissolved in yeast-water without adjusting the reaction.

Egg medium (Dorset).-Eggs are broken into a flask, and the yolks are broken with a platinum wire. Then the flask is gently shaken, so as to mix the yolks with the whites without causing foam to form. ro c.c. are distributed in culturetubes, and then the medium is hardened in a sloping position in the Koch inspissator for 2 successive days for 4 or 5 hours at $70^{\circ}$

Egg-yolk medium (Dorset).-Add 5-Io c.c. of sterile water to the yolks of 3 or 4 eggs, and then pursue the same course as in the other egg medium.

Egg-yolk medium (Capaldi).-A few loopfuls of egg-yolk are added to a tube of liquefied agar cooled to $45^{-} 47^{\circ}$.

Enriching solution for B. typhosus (Hoffmann and Fischer). - To peptone-meat-broth add I per cent. nutrose, 0.5 per cent. caffein, and I per cent. solution of crystal violet.

\section{Endo's medium.-}

Nutrient agar $(3 \%$ \% $) \ldots \ldots \ldots \ldots \ldots \ldots$. $\ldots \ldots o$ c.c.

Lactose........................

Alcoholic solution of fuchsin............ 5 c.c.

Sodium sulphite (Io\% solution).........25 c.c.

Sodium hydrate (ro\% solution)........... Io c.c.

Drigalski and Conradi's medium (Modified by Harris).Dextrose-free broth............... zooo c.c.

Nutrose..................... $20 \mathrm{~g}$.

Agar...................... 40 g.

Boil, dissolve, neutralize to phenolphthalein, autoclav at $120^{\circ}$ for 5 minutes. Clarify with whites of four eggs and filter. Then add- 


Lactose....................... $30 \mathrm{~g}$.

Litmus-solution....................260 c.c.

Crystal violet (o. I\% aqueous solution)..... 20 c.c. Tube and sterilize once in Arnold.

Uschinsky's non-proteid medium (Fraenkel's modification).-

Disodium hydrogen phosphate..........

Ammonium lactate.................. $6_{3} \mathrm{~g}$.

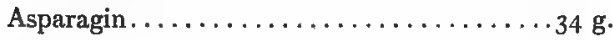

Sodium chlorid................... $50 \mathrm{~g}$.

Water .................... Io,000 c.c.

Phenol media.-I part carbolic acid is added to rooo parts medium.

Dog-blood serum.- Serum of dog's blood is coagulated in slanted tubes in the Koch inspissator for 3 hours at $75^{\circ}$.

Nitrate broth.-Add 5 parts of potassium nitrate to each rooo parts of ordinary broth.

Nitrate solution. -5 c.c. of 2 per cent. aqueous potassiumnitrate solution are added to a solution of $\mathbf{I} \mathrm{g}$. of peptone in rooo c.c. of water. 


\section{APPENDIX II \\ STAINING SOLUTIONS}

Delafield's hematoxylin-

Hematoxylin crystals .................

Alcohol. ........................

Ammonia alum.....................

Water...................... 400 c.c.

Glycerin.................... roo c.c.

Methyl alcohol .................. Ioo c.c.

Alum-hematoxylin-

I. Hematoxylin $\ldots \ldots \ldots \ldots \ldots \ldots \ldots \ldots, \mathbf{g}$ g.

Absolute alcohol................. I0o c.c.

2. Ammonia alum ..................

Water ...................... I00 c.c.

Mix I and 2 and add-

Glycerin..................... $88_{50}$ c.c.

Glacial acetic acid................. roo c.c.

Allow to stand for one month before using.

Bismarck brown-

Bismarck brown...................

Water ...................... roo c.c.

Safranin-

Safranin....................

Water ....................... roo c.c.

Carbolic thionin-blue (Nicollé)-

Thionin-blue ..................

Carbolic acid......................

Water....................... 100 c.c.

Alum carmin-

Alum ..................

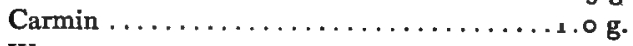

Water........................ roo c.c. 


Lithium-carmin (Orth)-

Carmin ......................

Saturated watery solution of lithium carbonate ..................... roo c.c.

Add a few crystals of thymol.

Kiihne's methylene-blue-

Methylene-blue.................. I.5 g.

Absolute alcohol .................. ro c.c.

Carbolic acid solution $(5 \%) \ldots \ldots \ldots \ldots$ I00 c.c.

Carbolic gentian-violet (Nicollé)

Gentian-violet (sat. alcoh. sol.) . . . . . . . . r ro c.c.

Carbolic acid.................... I g.

Water ........................ go c.c. 


\section{APPENDIX III}

\section{FROST'S CULTURE CHART (MODIFIED)}

Group

Name of organism.

Source, habitat, etc

References.

\begin{tabular}{|c|c|c|c|}
\hline MORPHOLOGICAL CHARACTERS: & 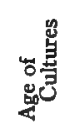 & 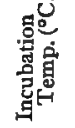 & Sketches \\
\hline 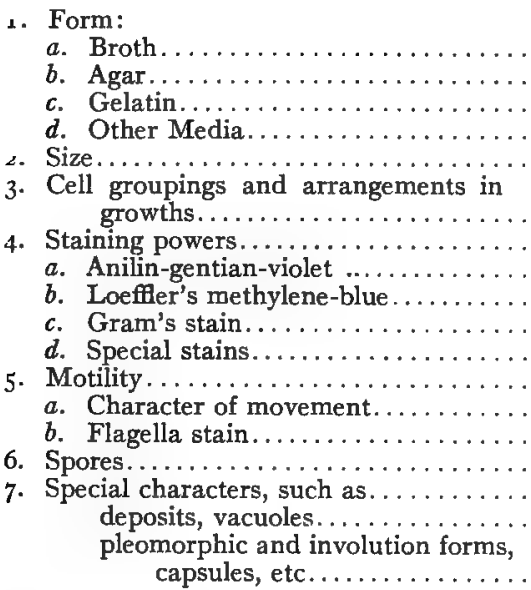 & & & \\
\hline
\end{tabular}

Physiological Characters

1. Relation to temperature

2. Relation to free oxygen

3. Relation to other agents, such as dessication, light, disinfectants, etc

This represents a model only. Open spaces and lines should be extended for practical use to allow sufficient room for descriptions. 


4. Pigment production

5. Growth in carbohydrate media:

a. Stab or shake culture.

b. Fermentation-tube: 1. Growth in bulb....2. In closed arm....

c. Percentage gas produced in: Dextrose.... Lactose...Saccharose After 24 hours. . . . . . . . $\ldots \ldots \ldots \ldots \mid \ldots \ldots \ldots \ldots \ldots$ After 48 hours.

Gas formula $\frac{\mathrm{H}}{\mathrm{CO}_{2}}$

d. Reaction in bulb.

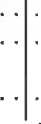

2. Closed arm

6. Acid or alkali production, in litmus milk.

7. Reduction of nitrates; to nitrites...... to ammonia

8. Indol production; 24 hours. . . . 48 hours. . ...4 days.

$$
\text { fecal odor; } 24 \text { hours. . . . } 48 \text { hours. . . . 4 days. }
$$

9. Enzym production; coagulative.... . proteolytic.... . diastatic.....

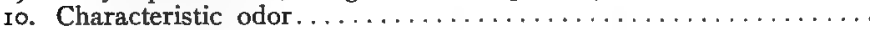

1r. Pathogenesis.

Cultural Characters of:

(I)

Gelatin

plate or

roll tube

a) Surface colonies

b) Deep colonies

(2)

Agar plate, or roll tube

a) Surface colonies

b) Deep colonies

$\left|\begin{array}{c|c|}\begin{array}{c}\text { Reaction of } \\ \text { Medium } \\ \text { Incubation } \\ \text { Temp. }\left({ }^{\circ} \mathrm{C}\right)\end{array} & \text { Description } \\ \hline & \end{array}\right|$


I36 LABORATORY GUIDE IN BACTERIOLOGY

Cultural Characters of:

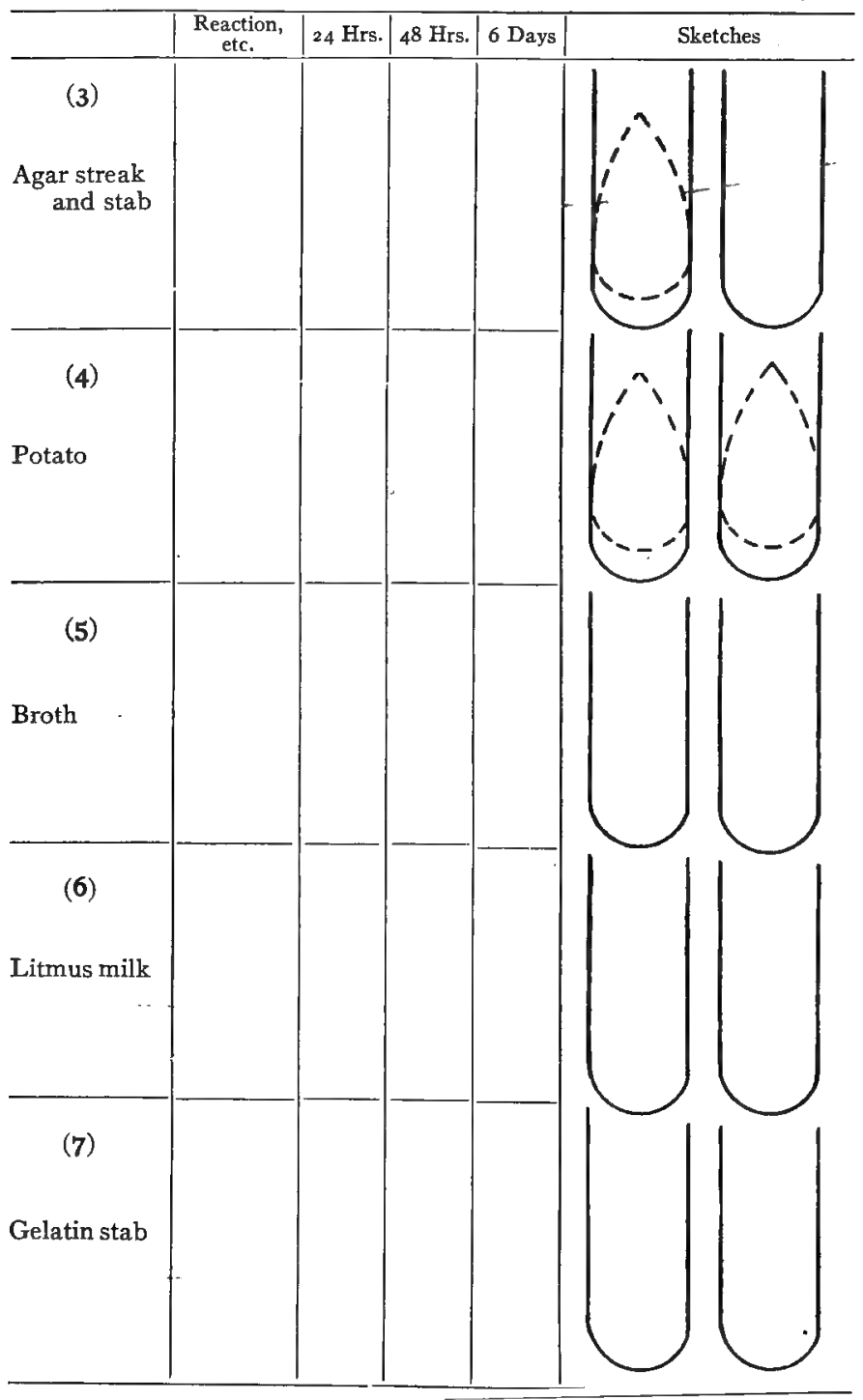




FROST'S CULTURE CHART

Cultural Characters of:

\begin{tabular}{c|c|c|c|c|c}
\hline & $\begin{array}{c}\text { Reaction, } \\
\text { etc. }\end{array}$ & 24 Hrs. & 48 Hrs. & 6 Days & \\
\hline (8) & & & & & \\
$\begin{array}{c}\text { Glucose-agar } \\
\text { or special } \\
\text { media }\end{array}$ & & & & & \\
\hline
\end{tabular}





\section{INDEX}

\begin{tabular}{|c|c|c|c|}
\hline \multicolumn{2}{|l|}{ 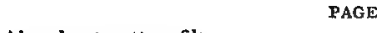 } & & PAGE \\
\hline Absorbent cotton filter & 17 & Bacillus mucosus capsulatus & 92 \\
\hline Acid, chromic & 8 & Bacillus oedematis maligni & 107 \\
\hline Acid-resisting bacili & 103 & Bacillus of bubonic plague & \\
\hline Actinomyces asteroides, bovis, et & tc. 106 & Bacillus of dysentery & 79.85 \\
\hline gar-agar & 11 & Bacillus of rabbit septicemia & \\
\hline Agar, beerwort & 128 & Bacillus paratyphosus & 79,84 \\
\hline Agar, bile-salt & 128 & Bacillus pestis & 97 \\
\hline Agar, blood & 128 & Bacillus prodigiosus & 69 \\
\hline Agar, glucose & 19 & Bacillus pseudo-diphthericus & 94 \\
\hline Agar, glycerin & I 28 & Bacillus pyocyaneus & 69 \\
\hline Agar, litmus-lactose & 127 & Bacillus smegmae & IO3 \\
\hline Agar, litmus-mannit & 127 & Bacillus subtilis & 98 \\
\hline Agar, neutral red & 127 & Bacillus tetani & 107 \\
\hline Agar, preparation of & I I & Bacillus tuberculosis & 103 \\
\hline Agar-slants & 39 & Bacillus typho & 79,85 \\
\hline Agglutination & 87 & Bacillus violaceus & 69 \\
\hline Air, bacterial examination of $37, \mathbf{I}$ & 14,118 & Bacillus xerosis & 94 \\
\hline cteria from & 37 & Bacteria, chromogenic & 69 \\
\hline e blood-serum & 128 & Bacteria from air & 37, I 18 \\
\hline Alun & 132 & Bacteria, pyogenic & 73,76 \\
\hline matoxylin & 132 & Bacteria, unknown & 112 \\
\hline & 65 & Bacterial examination of air & \\
\hline ic cultivation & 107 & 37,1 & I 14,118 \\
\hline Ana & 107 & Bacteria & II 4, I I \\
\hline Ana? & 82 & 2, of water & \\
\hline -violet & 30 & rmo & \\
\hline Ant & 98 & & \\
\hline Antl & 98 & a, Canada & \\
\hline sterilizer & 24 & & \\
\hline & 46 & & 128 \\
\hline & 20 & & 128 \\
\hline & 75 & filter & 46 \\
\hline acid_rea & 103 & & 128 \\
\hline Baci & 79 & broth & 129 \\
\hline Eapsulatus & 107 & brown & 132 \\
\hline & 98 & & 128 \\
\hline racis symptomatici & 107 & & 125 \\
\hline Baci & 107 & alkaline & 128 \\
\hline Bacillus cholerae suis & 79.84 & , dog's & I3I \\
\hline oacae & 79,89 & one broth) & \\
\hline & & & 128 \\
\hline & 97 & & $I 29$ \\
\hline seuche & 97 & & \\
\hline ineseuche & 97 & & 128 \\
\hline & & & 26 \\
\hline & 79,85 & & I3I \\
\hline Baci & 79,84 & $\mathrm{Br}$ & 25 \\
\hline us faecalis alcaligenes & 79,85 & 1, preparation of & \\
\hline is Gaertner's & 84 & & 27,79 \\
\hline & 103 & Brownian movement & \\
\hline ides & & Bubonic plague & \\
\hline Iërogenes & 79,92 & Buchner's anaërobic culture m & meth- \\
\hline & 103 & & 107 \\
\hline cillus mallei & 105 & Butter, bacilli in & 203 \\
\hline
\end{tabular}


Canada balsam

Capaldi's egg-medium

PAGE

Capsulated group

Capsule stain

Capsule stain, Friedländer's

Capsule stain, Welch's

Capsule stain, Welch's modified

Carbol-fuchsin

Carbolic gentian-violet

Carbolic thionin-blue

Carmin

Carmin, alum

Carmin, lithium

Casein

Caseinogen

Cedar oil

Cholera red reaction (see Indol)

Chromogenic group

Clarifying media

Cleaning glassware

Cleaning mixtures

Coagulation

Coagulative enzym

Colon bacillus

Colon group

Colonies, counting of

Colony

Condensation water

Conradi's medium

Cotton filter

Cotton filter, absorbent

Counting colonies

Culture-charts description

Culture media

Culture-tubes

Culture-tubes, plugging of

Culture-tubes, potato

Cultures, egg

Cultures, plate

Cultures, pure

Death-point, thermal

Decolorization of litmus

Delafield's hematoxylin

Description of colonies

Description of cultures

Dextrose (see under glucose)

Dextrose yeast water

Diagnosis of glanders (Strauss)

Diastase

Diastatic enzym

Dilution tables

Diphtheria bacillus

Diphtheria group

Diphtheria toxin

Directions for filling out culturecharts

Directions, general

Discontinuous sterilization

Disinfectants

Dog's blood-serum

Dorset's egg medium

Dorset's egg-yolk medium
44

130

92

76

77

77

I IO

30

I 33

I 32

43

132

I 33

64

64

35

IOI

60

13

8

64

64

79

79

1 6

30

40

$I_{30}$

47

I7

I 6

57

3, 4, I 4

3. 28

95

70

39

124

65

132

57

57

130

105

65

65

II 7

94

94

96

$6 \mathrm{I}$

2

24

122

I 31

130

130
Drigalski's medium

PAGE

Dung bacillus

Dunham's peptone solution

Dysentery bacillus

Egg cultures

Egg media

Ehrlich's anilin-gentian-violet

130

$\mathrm{IO}_{3}$

25

79,85

95

I 30

Elsner's medium

Endo's medium

30

I29

130

Enriching method of Schottelius IOI

Enriching solution (Hoffmann and Fischer)

Enzym

Enzym, coagulative

Enzym, diastatic

Enzym, production of

Enzym, rennet-like

Erlenmeyer flask

130

64

64

65

64

64

Examination, bacterial of air

Examination, bacterial of milk I I 4, I IO

Examination, bacterial of water II4

Fermentation-tube

Filament formation

Filling culture-tubes .

Filter, absorbent cotton

Filter, Berkefeld

5

Filter, paper

Filter rack 17

Filtering media 16

Filtering media by vacuum I7

Finkler and Prior's spirillum Ior

Flagella, staining of $\quad 86$

Flask, Erlenmeyer 5

Fowl cholera, bacillus of $\quad 97$

Friedländer's capsule stain $\quad 77$

Friedländer's pneumobacillus $\quad 92$

Frost's fermentation-chart $8 I$

Frost's culture-chart (modified) $\quad$ I34

Fruit-jar method of anaërobic cultivation

Fuchsin

I08

Gaertner's bacillus

Garden earth, inoculation of II I

Gas analysis $\quad 82$

Gas formula $\quad 82$

Gas generator IOQ

Gasometer (Frost's) $8 \mathrm{r}$

Gelatin, beerwort I28

Gelatin, glucose $\quad$ I28

Gelatin, liquefaction of $\quad 64$

Gelatin, litmus-lactose $\quad$ I27

Gelatin, peptone 23

Gelatin, whey $\quad$ r28

General directions 2

Gentian-violet 30

Gentian-violet, carbolic $\quad 133$

Germination of spores 5 I

Glanders

Glassware, cleaning 


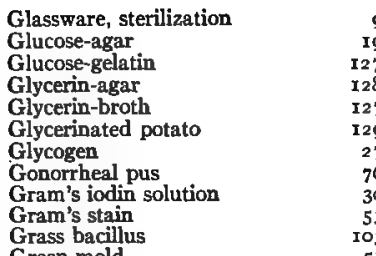

Green mold 5 I

Gruber-Widal test for typhoid fever 87

Gypsum blocks

Hand-lens

Hanging-drop

Hay-infusion

Hematoxylin alum

Hematoxylin, Delafield's

Hemorrhagic septicemia group

Hill's test-rods

Hoffmann and Fischer's enriching method

Hog-cholera group

Hot-air sterilizer

Hydrogen gas-generator

Immersion oil

Impression preparation

Incubator

Indol

Indol test

Infection, phenomena of

Influence of disinfectants

Influence of moist heat

Influence of sunlight

Infusion, hay

Inoculation (see various headings)

Intermediate group

Intermittent sterilization

Intestinal group

Intramuscular inoculation

Intraperitoneal inoculation

Intravenous inoculation

Involution forms

Iodin solution, Gram's

Isolation of unknown bacteria

Jar, Novy

Klatschpräparat

Kühne's methylene-blue

Labeling media

Laboratory rules

Lactose-litmus-agar

Lactose-litmus-gelatin

Liquefaction of casein

Liquefaction of gelatin

Lithium carmin

Litmus-broth

Litmus, decolorization of

Litmus-lactose-agar

Litmus-lactose-gelatin

Litmus-mannit-agar
PAGE

9

127

128

I27

129

27

76

53

3

50

5

42

129

132

132

97

122

130

79,84

9

rog

34
98

55

63

85

45

122

I 24

I 23

I29

79,84

79,84

24

79

IO2

92

73

89

30

112

I08

98

133

20

I

I 27

127

64

64

133

128

65

I 27

I 7

127
Litmus milk

PAGE

Loeffler's flagella stain $\quad 86$

Loeffler's methylene-blue 30

Mac Conkey's bile-salt agar $\quad$ I28

Mac Conkey's bile-salt broth 129

Magnifier

Mannit litmus agar $\quad 127$

Meat broth $\quad 26$

Meat-press 26

Media, adjusting reaction of $\quad r_{4}$

Media, adjusting weight of

Media, clarifying 13

Media, filling in tubes

Media, filtering I4

Media, filtering by vacuum I8

Media, phenol I3I

Media, preparation of II

Media, preservation of $\quad 28$

Media, reaction of 12

Media, special 125

Media, standard methods

Medium, bread-paste 128

Medium, Drigalski-Conradi's 130

Medium, Elsner's 129

Medium, Endo's 130

Medium, Uschinsky's I3I

Medium, wine-must

Method of describing cultures $\quad 39$

Method of inoculation of media 57

Methylene-blue, Kühne I

Methylene-blue, Loeffler 30

Micrococcus gonorrhoeae $\quad 76$

Micrococcus intracellularis meningitidis

Micrococcus lanceolatus $\quad 76$

Micrococcus melitensis I05

Micrococcus tetragenus $\quad \mathbf{7 3}$

Micrococcus zymogenes $\quad 76$

Microscope, description of 31

Milk, acid-resisting bacilli in $\quad \mathrm{IO4}$

Milk, bacterial, examination of I I 4 , I 19

Milk, litmus 28

Milk, pasteurization of I 9

Milk, sterilization of IIg

Moeller's grass bacillus 103

Moeller's spore stain $\quad 98$

Moist heat, influence of $\quad$ I24

Moist heat, sterilization by 20,24

Mold, green 5 I

Mold spores 39

Molds 49

Molds, staining of 50

Molecular movement $\quad 43$

Mordant 86

Mouse, inoculation of 78

Mouse-holder $\quad 78$

Muscle-sugar $\quad 27$

Needles, platinum 4

Neisser's stain $\quad 94$

Neutral red agar $\quad 127$ 


\begin{tabular}{|c|c|c|c|}
\hline & PAGE & & PAGE \\
\hline Nicolle's carbolic gentian-violet & 133 & Reaction of media & 14 \\
\hline Nicolle's carbolic thionin-blue & $\mathbf{x}^{32}$ & Rennet-like enzym & 64 \\
\hline Nitrate broth & .31 & Rinderpest & 97 \\
\hline Nitrate solution & I3 I & Routine study & 53 \\
\hline Nitrites & 63 & Saccharomyces cerevisiae & 49 \\
\hline Nitrites, test for & 83 & Safranin & 132 \\
\hline Nitroso-indol reaction & IOI & Sarcina lutea & 69 \\
\hline Normal solution & 13 & Schottelius' enriching method & roI \\
\hline Novy jar & 108 & Schweineseuche & 97 \\
\hline Oil, immersion & 34 & Soap-powder & \\
\hline Orth's lithium carmin & I33 & Solution, Dunham's peptone & 25 \\
\hline Parietti's solution & 129 & Solution, litmus & 127 \\
\hline Park's anaërobic culture method & 107 & Solution, nitrate & $13 \mathrm{I}$ \\
\hline tion of milk & II & Solution, normal & I3 \\
\hline Pedesis & 43 & Solution, Parietti's & 129 \\
\hline glaucum & $5 \mathbf{I}$ & Solutions, staining & 30,132 \\
\hline Peptone broth & 25 & Special media & 125 \\
\hline latin & 23 & Spirillum cholerae asiaticae & IOI \\
\hline Peptone solution, Dunham's & 25 & Spirillum Metchnikovi & IOI \\
\hline Pept & 64 & Spirillum of Finkler and Prior & IOI \\
\hline Petri & 5 & Spirillum tyrogenum & IOI \\
\hline Phe & I3I & Spore-stain & 98 \\
\hline lein & 12 & ination of & $5 I$ \\
\hline infection & 45 & & 20 \\
\hline f sterilization & 45 & of yeast & $5 \mathrm{I}$ \\
\hline Pigm & 71 & ef molds & 39 \\
\hline Plat & 70 & inder's capsule & 77 \\
\hline Plati & 4 & Stain, Welch's capsule & 77 \\
\hline re-tubes & 8 & Stain, Welch's capsule modified & IIo \\
\hline Pne & 92 & n, Gram's & 53 \\
\hline Poc & 96 & a's flagella & 86 \\
\hline Polar & 5,97 & St: & 94 \\
\hline rinated & 129 & ration & 44 \\
\hline aration of & 27 & esisting bacilli & 103 \\
\hline Pota & 28 & & 86 \\
\hline Prepe & 53 & Sta & $5 I$ \\
\hline Prep & 98 & & 85,97 \\
\hline Prep & II & ag solutions & 30,132 \\
\hline Prep & 25 & Staining spores & 98 \\
\hline Prep & 25 & Standard, Fuller's & \\
\hline of culture media & II & Standard method of & ring \\
\hline $\begin{array}{l}\text { Preparation of Dunham's pepton } \\
\text { solution }\end{array}$ & & & \\
\hline & 25 & Staphylococcus pyogenes albus & \\
\hline Prep & 23 & ylococcus pyogenes aureus & 73 \\
\hline Preparation of glucose-agar & I9 & & 65 \\
\hline Preparation of stains (see variou & & Ste & 24 \\
\hline $\begin{array}{l}\text { headi } \\
\text { Prepara }\end{array}$ & & $\begin{array}{l}\text { Ster } \\
\text { Ster }\end{array}$ & \\
\hline $\begin{array}{l}\text { Prepara } \\
\text { Prepar }\end{array}$ & $\begin{array}{l}25 \\
27\end{array}$ & $\begin{array}{l}\text { uous } \\
\text { ent }\end{array}$ & $\begin{array}{l}24 \\
24\end{array}$ \\
\hline Pre & 44 & sswar & \\
\hline Prese & II & & rio \\
\hline Proteol & 64 & 1, phenomena of & 45 \\
\hline & 80 & & \\
\hline Pure & & Sterilizer, Arnold steam & \\
\hline orrheal & 76 & Strauss method of diagnosis & of \\
\hline & 72 & & 105 \\
\hline group $\mathbf{A}$ & 73 & Streptococcus pyogenes & 73 \\
\hline & 76 & & 53 \\
\hline Rab & 97 & Subcutaneous & 85 \\
\hline & 83 & Sug & 27,79 \\
\hline & 83 & & 106 \\
\hline Reaction, nitroso-indol & IOI & Sunlight, influence of & 123 \\
\hline
\end{tabular}


Tables, dilution

Tetanus toxin

PAGE

PAGE

Test for indol

Test for nitrites

Test-rods, Hill's

Thermal death-point

17

Water of condensation

40

110 Welch's capsule stain 77

83 Welch's capsule stain modified I Io

Thermostat

Whey

64

122

Whey-gelatin

128

124 Whey litmus 128

Thionin-blue

Torulæ

Tube, culture

Tube, fermentation

Tube, potato culture

Tubes, plugging of

Typhoid-dysentery group

Widal test (see Gruber-Widal test)

55

132

Wine-must

49 Winogradsky's solution 129

129

4 Xylol

Yeast water

28 Yeast water, dextrose

33,35

8

Yeasts

130

79,85

Yeasts, budding of

130

Uschinsky's medium

Yeasts, spores of

$39,49,51$

Water, bacterial examination of 




